MKPH-T-98-3

\title{
Electroweak Processes in Few-Nucleon Systems ${ }^{* \dagger}$
}

\author{
Hartmuth Arenhövel \\ Institut für Kernphysik \\ Johannes Gutenberg-Universität \\ D-55099 Mainz, Germany
}

July 2, 2021

\begin{abstract}
After a brief introduction into the basic ingredients of electroweak theory as a spontaneously broken local, non-Abelian gauge symmetry, the general properties of the electromagnetic current and two-photon operators are discussed. In particular, the consequences of gauge invariance and the resulting low energy theorems are reviewed. The multipole decomposition of the current operators and the general Siegert theorem are presented. The specific forms of vector and axial one-nucleon currents are given, together with lowest order $\pi$ meson exchange and isobar currents as well as meson production currents. A brief overview is given on the most important one- and two-boson processes. Electron scattering in the one-boson-approximation is then considered in greater detail. Formal expressions of the cross section for inclusive and exclusive processes are given, including parity violating contributions from $\gamma-Z$ interference as well as from parity violating components in the hadronic wave function. Specific electromagnetic reactions on the deuteron are then discussed with respect to the influence of meson exchange currents, isobar configurations in the deuteron groundstate, relativistic contributions and the role of $\pi$-meson retardation. Furthermore, recent results on coherent and incoherent $\pi$ - and $\eta$-photoproduction are presented as well as a discussion of the Gerasimov-Drell-Hearn sum rule and the effect of a parity violating deuteron component on inclusive electron scattering off the deuteron for quasifree kinematics. The review closes with a summary and a brief outlook.
\end{abstract}

\footnotetext{
${ }^{*}$ Lectures held at the Int. Workshop on Few-Body Problems in Nuclear Physics and Related Fields, ECT*, Trento, Italy, September 8-27, 1997

${ }^{\dagger}$ Supported by the Deutsche Forschungsgemeinschaft (SFB 201)
} 


\section{Contents}

1 Introduction $\quad 3$

2 The Electroweak Interaction as a Non-Abelian Gauge Theory 4

2.1 Abelian and Non-Abelian Gauge Symmetry . . . . . . . . . . . . . . . . . 4

2.2 Spontaneous Symmetry Breaking and Mass Generation . . . . . . . . . . . 8

2.3 The Electroweak Lagrangian of the Standard Model . . . . . . . . . . . . . . 10

3 Properties of the Electromagnetic Operators $\quad 11$

3.1 Gauge Conditions for the Electromagnetic Operators . . . . . . . . . . . . . 11

3.2 Low Energy Theorems . . . . . . . . . . . . . . . . . . . . . . . 11

3.3 Multipole Decomposition . . . . . . . . . . . . . . . . . 12

3.4 Generalized Siegert Theorem and Siegert Operators _ . . . . . . . . . . . 13

4 Models for the Electromagnetic Operators $\quad 14$

4.1 One-Body Currents . . . . . . . . . . . . . . . . . . . . . . 14

4.2 Two-Body Meson Exchange Currents . . . . . . . . . . . . . . . . . . . 15

4.3 Isobar Currents . . . . . . . . . . . . . . . . . . . . . 16

4.4 Meson Production Currents . . . . . . . . . . . . . . . . . . . 17

4.5 Relativistic Contributions . . . . . . . . . . . . . . . . . . 17

5 Basic Electroweak Processes $\quad 18$

5.1 One-Boson Processes . . . . . . . . . . . . . . . . . . . . 18

5.2 Two-Boson Processes . . . . . . . . . . . . . . . . . . . . . . 19

5.3 Cross Section for Electron Scattering . . . . . . . . . . . . . . 20

6 Selected Reactions on the Deuteron $\quad 24$

6.1 Electromagnetic Break-up . . . . . . . . . . . . . . . . . . 24

6.1.1 Backward Electrodisintegration near Threshold . . . . . . . . . . . . 24

6.1.2 Signature of a $\Delta \Delta$ Component in Electrodisintegration . . . . . . . . 27

6.1.3 Heavy Meson Exchange and Consistent Relativistic Effects in Photodisintegration below Pion Threshold . . . . . . . . . . . . . 28

6.1.4 Pion Retardation in Photodisintegration above $\pi$ Threshold . . . . . . 31

6.2 Photoproduction of Mesons . . . . . . . . . . . . . . . . 33

6.2.1 Coherent Pion Photoproduction in the $\Delta(1232)$ Region . . . . . . . 34

6.2.2 Coherent Eta Photoproduction in the $S_{11}(1535)$ Region . . . . . . . . 37

6.2.3 Final State Interaction in Incoherent Eta Photoproduction . . . . . . 39

6.3 The Gerasimov-Drell-Hearn Sum Rule . . . . . . . . . . . . . . . . . . 42

6.3.1 The GDH Sum Rule for the Nucleon . . . . . . . . . . . . . . . . . 44

6.3.2 The GDH Sum Rule for the Deuteron . . . . . . . . . . . . . . 45

6.4 Parity Violation in Quasifree Electron Scattering . . . . . . . . . . . . 49

7 Conclusions and Outlook $\quad 52$

$\begin{array}{ll}\text { References } & 53\end{array}$ 


\section{Introduction}

One of the major goals of present day research in the field of medium energy physics is to clarify the role of effective subnuclear degrees of freedom (d.o.f.) in terms of nucleon, meson and isobar d.o.f., and their relationship to the underlying more fundamental d.o.f. of quantum chromodynamics (QCD). In other words, the question is, what is the connection of such effective d.o.f. to the presumably underlying quark-gluon dynamics of QCD. A large fraction of our present knowledge about the internal constitution of nuclei or more general of hadrons has been obtained from electromagnetic reactions. Indeed, throughout the history of nuclear and elementary particle physics, starting with the discovery of the nucleus by Coulomb scattering of $\alpha$-particles off a gold foil in 1909 up to the discovery of point-like objects as internal constituents in the proton in deep inelastic lepton scattering in 1967, the electromagnetic probe has provided us with a wealth of important information on the internal structure of hadronic systems.

The salient features of the electromagnetic (e.m.) interaction, which make it such a valuable tool, are well known and may be summarized as follows:

- The e.m. interaction is already known from classical physics (Maxwell theory).

- The e.m. interaction fulfills the basic requirements of a fundamental interaction, i.e., it incorporates the principles of special relativity and represents the simplest case of a gauge theory, namely an Abelian gauge theory.

- The e.m. interaction is weak enough, characterized by the small fine structure constant $\alpha=1 / 137$, so that in most cases lowest order perturbation theory is sufficient, allowing a simple interpretation of observables in terms of charge and current density matrix elements.

Another important source of information is provided by reactions involving the weak interaction as, for example, manifest in beta decay. Originally, the weak interaction was considered as an independent fundamental force, and it was only much later that its unification with electromagnetism was established, although already Fermi intuitively had formulated his famous four-fermion theory of beta decay along the scheme of the e.m. interaction. Indeed, already in 1935, Yukawa [Yuk 35] had hypothesized a heavy boson as mediator of the weak interaction, which at low energy would effectively give the point coupling of the Fermi theory. The various stages of the historical development of the theory of the weak interaction may be summarized as follows:

- Discovery of radioactivity by Becquerel in 1896, and subsequent identification of beta rays as electrons.

- Postulate of the neutrino by Pauli in 1930 in order to save the energy conservation law in beta decay.

- First formulation of a theory for beta decay by Fermi in 1934 as a current-current interaction, modelled in analogy to the e.m. case.

- Discovery of parity violation in beta decay in 1957 by Wu, Ambler, Hayward, Hoppes, Hudson [WuA 57] after a critical analysis of the status of experimental evidence for parity conservation in weak processes by Lee and Yang in 1956 [LeY 56]. 
- Formulation of the $(V-A)$-theory by Gell-Mann and Feynman [FeG 58] and independently by Sudarshan and Marshak [SuM 58] in 1958. Postulate of universality of the current-current interaction [FeG 58], and renewed hypothesis of an intermediate vector boson as mediator of the weak interaction.

Finally, about 30 years ago, e.m. and weak interactions were unified. The essential steps towards this unification were

- Study of a local non-Abelian gauge symmetry by Yang and Mills [YaM 54] in 1954 for the case of an isospin $S U(2)$ symmetry. It is the simplest case of what now is called in general a Yang-Mills theory.

- Introduction of spontaneous breaking of a local gauge symmetry as mass generating mechanism for vector bosons by Higgs, Kibble and others in 1964-66 (massive YangMills theory).

- Unification of e.m. and weak interactions via a spontaneously broken $S U(2)_{L} \times U(1)$ gauge symmetry by Glashow, Weinberg and Salam in 1967.

- Proof of renormalizability of electroweak theory by 't Hooft in 1971.

I will begin these lectures by reviewing first the essential ideas of the unified electroweak theory as a spontaneously broken non-Abelian gauge theory leaving out all the finer details which may be found in appropriate textbooks, e.g. in [BeB 83, Hua 92, Nac 86, Wei 95]. In Sect. 3, the properties of the e.m. interaction operators, gauge conditions, low energy theorems, multipole decomposition and generalized Siegert operators will be briefly discussed. Explicit expressions for the current operators will be collected in Sect. 4. Then in Sect. 5, an overview on basic electroweak processes is given. A variety of specific reactions on the deuteron will be considered in Sect. 6 like, e.g., photo- and electrodisintegration, coherent and incoherent meson photoproduction, the Gerasimov-Drell-Hearn sum rule and parity violation in electron scattering. I will close with some conclusions and an outlook.

\section{The Electroweak Interaction as a Non-Abelian Gauge The- ory}

As already mentioned, the two basic ingredients for the unified description of e.m. and weak processes are (i) a non-Abelian gauge symmetry, and (ii) spontaneous symmetry breaking.

\subsection{Abelian and Non-Abelian Gauge Symmetry}

In order to illustrate the fact how the postulation of invariance under a local gauge symmetry leads to the existence of an interacting massless vector field, the gauge field, let me consider first the simplest case of an Abelian gauge symmetry for a free complex scalar field $\Phi(x)$ having a Lagrangian

$$
\mathcal{L}_{0}\left(\Phi, \partial_{\mu} \Phi\right)=\partial^{\mu} \Phi^{*} \partial_{\mu} \Phi-V\left(\Phi^{*} \Phi\right) .
$$

This Lagrangian is evidently invariant under a global $U(1)$ gauge transformation (phase transformation)

$$
\begin{aligned}
\Phi & \Longrightarrow U(\theta) \Phi, \quad U(\theta)=e^{-i \theta}, \\
\partial_{\mu} \Phi & \Longrightarrow U(\theta) \partial_{\mu} \Phi,
\end{aligned}
$$


where $\theta$ is a real constant parameter. According to the Noether theorem, this invariance then leads to a conserved vector current

$$
\partial^{\mu} j_{\mu}=0 \quad \text { with } \quad j_{\mu}=i \Phi^{*} \stackrel{\leftrightarrow}{\partial_{\mu}} \Phi
$$

It is very useful for the understanding of a local gauge symmetry to give a geometrical interpretation of this phase transformation [BeB 83]. The fact, that the field is complex, may be visualized by representing it as a vector in a complex plane, an internal two-dimensional space, which is attached to each space-time point. In principle, one could imagine to assign different frames to these internal spaces at different space-time points. In this case, however, one could not compare vectors at different points anymore. In other words the affine connection would be lost. In fact, in writing a complex field amplitude, the tacit assumption is made, that at different points the internal frames can be identified establishing thus the affine connection, which is necessary for taking the derivative. Therefore, a global gauge transformation can be viewed as a global rotation of the internal frame at each space-time point by the same angle $\theta$ so that the affine connection is not lost (see Fig. 2.1).

This interpretation is important for the understanding of a local gauge transformation which is defined by

$$
\Phi(x) \Longrightarrow U(\theta(x)) \Phi(x)
$$

where $\theta(x)$ is a real arbitrary parameter function of $x$. The motivation for considering such local transformations of the internal frames is, that the physics should not depend on the local choice of the internal frame [Wey 29]. Under such a transformation, the Lagrangian is no longer invariant, because it does not commute anymore with the derivative, instead one has

$$
\partial_{\mu} \Phi \Longrightarrow U(\theta(x))\left(\partial_{\mu} \Phi(x)-i \partial_{\mu} \theta(x) \Phi(x)\right)
$$

This fact can be understood easily in the geometric interpretation of the gauge transformation, because the internal coordinate systems are now rotated by an arbitrary angle $\theta(x)$, i.e., differently at different points which means that the affine connection is lost. In other words, the field vectors at different points cannot be compared to each other anymore and thus the derivative is no longer well defined.

At this point the gauge field comes into play, because this problem can be cured by introducing a new vector field $A_{\mu}(x)$, by which one defines a parallel displacement of internal vectors from one point to another so that internal vectors at different points can be compared.

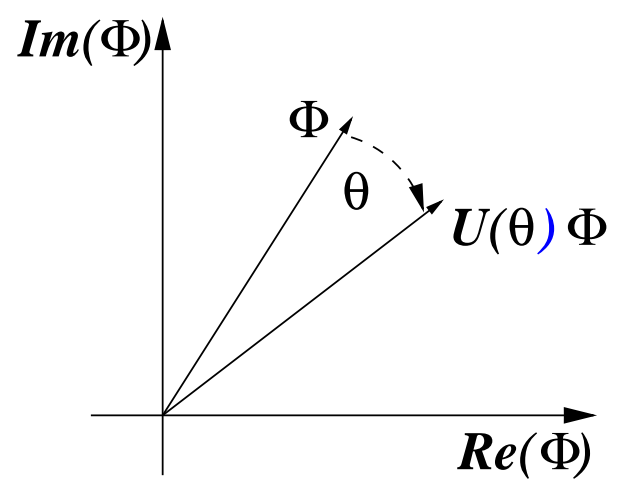

Fig. 2.1 Visualization of a phase transformation as rotation in an internal space. 
For this reason, Weyl has coined the name "gauge field" for $A_{\mu}(x)$. It is also subject to a certain gauge transformation as is specified below. The parallel displacement of a field vector at point $x$ to the point $x+d x$ via the gauge field is defined by

$$
{ }^{x+d x} \Phi(x):=\left(1-i e A_{\mu}(x) d x^{\mu}\right)^{x} \Phi(x),
$$

where ${ }^{x} \Phi(x)$ denotes the representation of $\Phi(x)$ in the internal basis at point $x$ and correspondingly ${ }^{x+d x} \Phi(x)$ its representation in the internal basis at point $x+d x$, i.e., the same internal vector in different internal coordinate systems. Here, $e$ is a coupling constant which will characterize the strength of the interaction of the gauge field with the given scalar field.

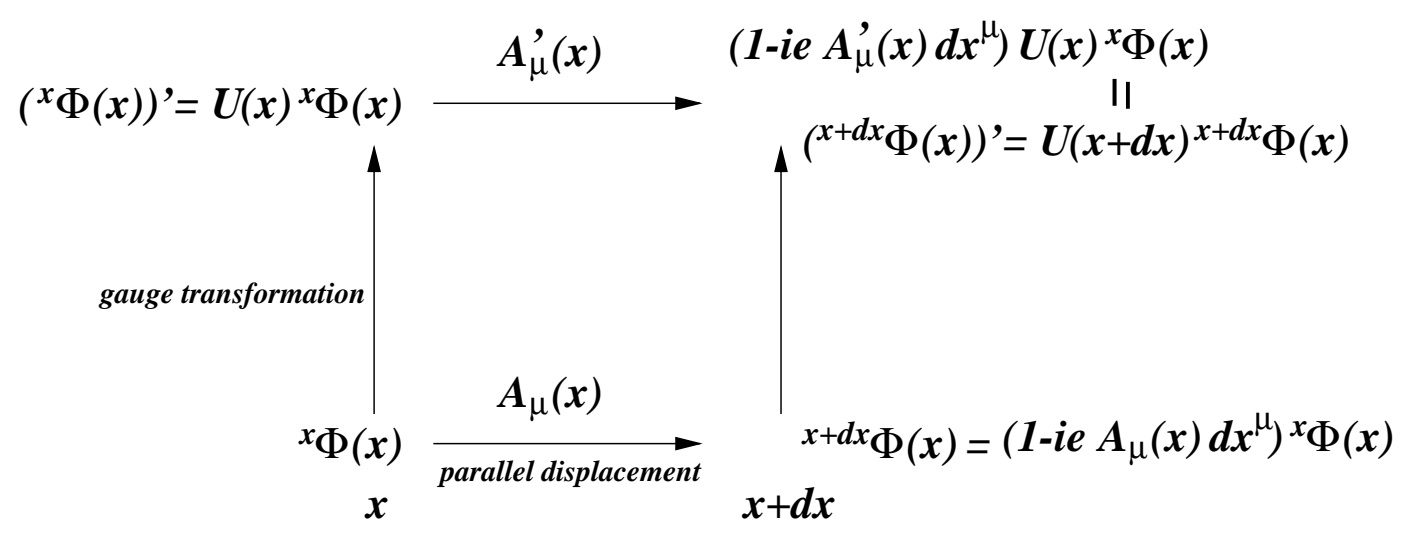

Fig. 2.2 Parallel displacement and local gauge transformation.

In order to have a meaningful definition of this parallel displacement, i.e., independent from any local gauge transformation, one has to require that one gets the same result if one first makes a gauge transformation and then a parallel displacement or vice versa (see Fig. 2.2 ). This condition then leads to the well known transformation law for the gauge field $A_{\mu}(x)$

$$
A_{\mu}(x) \quad \Longrightarrow \quad A_{\mu}^{\prime}(x)=A_{\mu}(x)+\frac{1}{e} \partial_{\mu} \theta(x) .
$$

With the help of the gauge field, one then introduces the covariant derivative

$$
D_{\mu}:=\partial_{\mu}+i e A_{\mu}(x)
$$

which transforms under a local gauge transformation according to

$$
D_{\mu} \phi(x) \Longrightarrow U(\theta(x)) D_{\mu} \phi(x),
$$

i.e., $D_{\mu}$ commutes with the gauge transformation. Replacing then in (2.1) the ordinary derivatives by the covariant derivatives, yielding the Lagrangian

$$
\mathcal{L}_{0}\left(\phi, D_{\mu} \phi\right)=D^{\mu} \phi^{*} D_{\mu} \phi-V\left(\phi^{*} \phi\right),
$$

one has achieved invariance of $\mathcal{L}_{0}$ under a local gauge transformation.

The geometrical meaning of the covariant derivative becomes clear if one compares the field at two neighbouring points $x$ and $x+d x$ with the help of the parallel displacement (Fig. 2.3). One readily notices that the covariant derivative mediates the connection of the field at two neighbouring points. In other words, the difference of the field vectors at the points $x+d x$ and $x$ evaluated at the point $x$ is just given by the covariant derivative.

$$
{ }^{x} \Phi(x+d x)-{ }^{x} \Phi(x)=D_{\mu}^{x} \Phi(x) d x^{\mu} .
$$




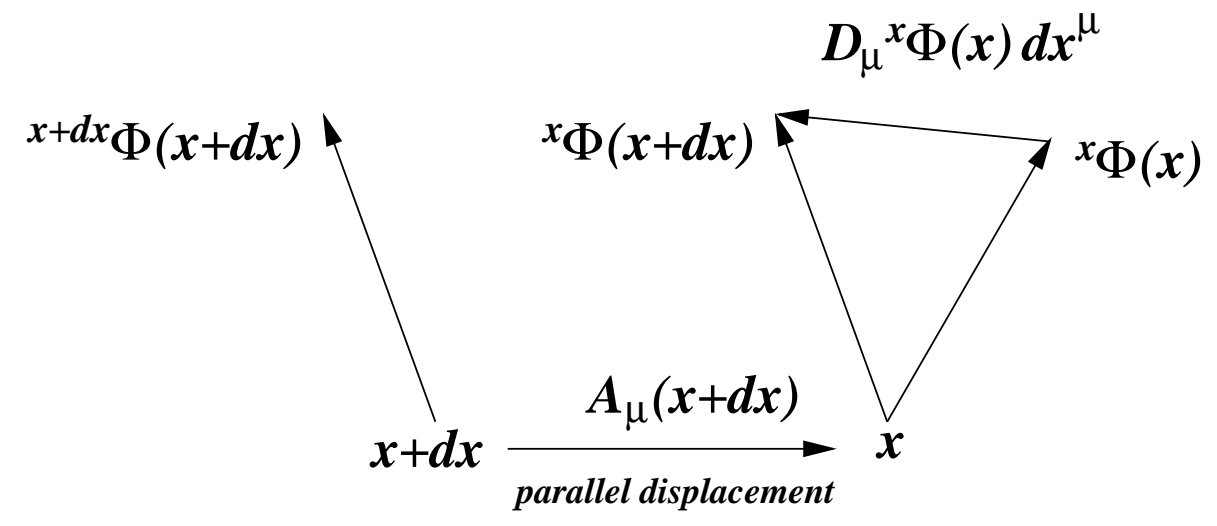

Fig. 2.3 Geometrical interpretation of the covariant derivative.

For finite distances, the connection depends on the path, because the components of the covariant derivative do not commute, instead one has

$$
\begin{aligned}
{\left[D_{\mu}, D_{\nu}\right] } & =i e\left(\partial_{\mu} A_{\nu}-\partial_{\nu} A_{\mu}\right) \\
& =i e F_{\mu \nu}
\end{aligned}
$$

where the field strength tensor $F_{\mu \nu}$ is defined by the last line. Obviously, the field strength tensor is invariant under the local gauge transformations of (2.8).

Until now, the gauge field was introduced as an external field. Thus, the final step is to introduce the gauge field dynamics by a gauge invariant Lagrangian for the free gauge field

$$
\mathcal{L}_{g . f .}=-\frac{1}{4} F_{\mu \nu} F^{\mu \nu}
$$

which describes massless vector bosons. A mass term would violate the gauge symmetry. The total Lagrangian then reads

$$
\mathcal{L}\left(\Phi, \partial_{\mu} \Phi, A_{\mu}, F_{\mu \nu}\right)=-\frac{1}{4} F_{\mu \nu} F^{\mu \nu}+D^{\mu} \Phi^{*} D_{\mu} \Phi-V\left(\Phi^{*} \Phi\right) .
$$

It can be interpreted as describing the quantum electrodynamics (QED) of charged scalar particles. This simple example illustrates nicely the essential idea that the postulate of a local gauge symmetry leads to the introduction of a massless vector field, the gauge field, as a fundamental interaction. It is well known that gauge invariance is essential in order to have a renormalizable theory.

I will now consider the generalization to gauging a non-Abelian internal symmetry group as is the case, for example, for the weak isospin $S U(2)_{L}$ of the electroweak interaction or the color $S U(3)$ of QCD. Let us take a Yang-Mills field which consists, for example, of $n$ complex components $\psi_{c}$

$$
\psi=\left(\begin{array}{c}
\psi_{1} \\
\vdots \\
\psi_{n}
\end{array}\right),
$$

which form the fundamental representation of an internal $S U(n)$ symmetry group, possessing $l=n^{2}-1$ generators $\hat{T}_{a}(n \times n$ matrices $)$, where $a=1, \ldots, l$ labels the generators. In the following, the Einstein convention for summation over equal indices is also used for the index $a$. 
A local gauge transformation has then the form

$$
\psi \Longrightarrow \hat{U}(x) \psi
$$

where now

$$
\hat{U}(x)=e^{-i \theta_{a}(x) \hat{T}_{a}}
$$

is a unitary $n \times n$ matrix. The non-Abelian character of the symmetry group is reflected by the commutation relations of the generators

$$
\left[\hat{T}_{a}, \hat{T}_{b}\right]=i f_{a b}^{c} \hat{T}_{c}
$$

where $f_{a b}^{c}$ denote the structure constants of the Lie algebra of the underlying symmetry group.

Invariance of the Lagrangian under such a transformation is achieved by introducing in analogy to the Abelian case a vector field $\hat{A}_{\mu}(x)$ which now is a $n \times n$ matrix field

$$
\left(\hat{A}_{\mu}(x)\right)_{c^{\prime} c}=A_{\mu}^{a}(x)\left(\hat{T}_{a}\right)_{c^{\prime} c} .
$$

Thus the number of independent gauge fields $A_{\mu}^{a}(x)$ equals the number of generators. The gauge field transforms according to

$$
\hat{A}_{\mu}(x) \Longrightarrow \hat{U}(x)\left(\hat{A}_{\mu}(x)-\frac{i}{g} \partial_{\mu}\right) \hat{U}^{-1}(x),
$$

and correspondingly, the covariant derivative becomes a $n \times n$ matrix operator

$$
\left(\hat{D}_{\mu}\right)_{c^{\prime} c}=\delta_{c^{\prime} c} \partial_{\mu}+i g A_{\mu}^{a}(x)\left(\hat{T}_{a}\right)_{c^{\prime} c},
$$

where $g$ denotes a coupling constant to be determined from experiment. Also the field strength tensor is represented by a $n \times n$ matrix

$$
\begin{aligned}
\hat{F}_{\mu \nu}(x) & =\frac{1}{i g}\left[\hat{D}_{\mu}, \hat{D}_{\nu}\right] \\
& =F_{\mu \nu}^{a}(x) \hat{T}_{a},
\end{aligned}
$$

where

$$
F_{\mu \nu}^{a}(x)=\partial_{\mu} A_{\nu}^{a}(x)-\partial_{\nu} A_{\mu}^{a}(x)-g f_{b c}^{a} A_{\mu}^{b}(x) A_{\nu}^{c}(x) .
$$

The essential difference to an Abelian gauge group lies in the fact, that the field strength tensor contains terms quadratic in the gauge field. Consequently, already the free gauge Lagrangian,

$$
\mathcal{L}_{g . f .}=-\frac{1}{4} F_{\mu \nu}^{a} F^{a, \mu \nu}
$$

describes self-interactions of the gauge field which are absent in the Abelian case. They are driven by the three- and four-gluon vertices in Fig. 2.4.

\subsection{Spontaneous Symmetry Breaking and Mass Generation}

A very important step towards the unification of the e.m. and weak interactions was the observation that the massless gauge bosons can acquire effectively masses by the AndersonHiggs-Kibble mechanism of spontaneous symmetry breaking via the coupling to massless scalar Higgs-fields. I will illustrate this for the case of a three-component gauge field $\vec{A}_{\mu}$ 

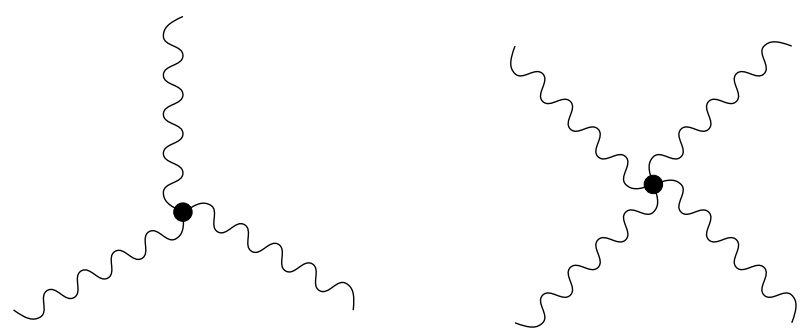

Fig. 2.4 Basic three- and four-gluon diagrams describing gluon self-interactions.

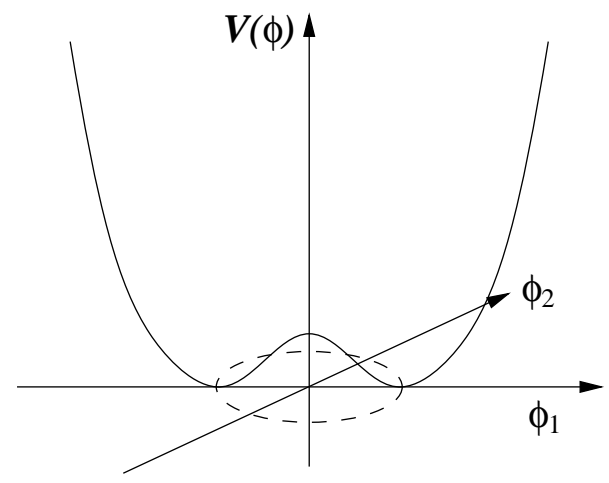

Fig. 2.5 The Higgs potential.

(combined as a vector) which arises in gauging a $S U(2)$ internal symmetry. In this case, the Higgs-field consists of two complex scalar fields constituting a $S U(2)$ doublet

$$
\phi=\left(\begin{array}{c}
\phi_{1} \\
\phi_{2}
\end{array}\right)
$$

with a Lagrangian of the form

$$
\mathcal{L}=-\frac{1}{4} \vec{F}_{\mu \nu} \cdot \vec{F}^{\mu \nu}+D^{\mu} \phi^{\dagger} D_{\mu} \phi-V(\phi)
$$

where the covariant derivative is given by

$$
D_{\mu}=\partial_{\mu}+\frac{i g}{2} \vec{A}_{\mu}(x) \cdot \vec{\tau} .
$$

The potential, which has first been considered in the Ginzburg-Landau theory of superconductivity and which later has also been used in the Goldstone model for the spontaneous breaking of a global symmetry, is given by the ansatz

$$
V(\phi)=\frac{\lambda}{4}\left(\phi^{\dagger} \phi-\frac{\mu^{2}}{\lambda}\right)^{2}, \quad \frac{\mu^{2}}{\lambda}>0 .
$$

It is illustrated in Fig. 2.5.

This Lagrangian is invariant under the local gauge transformation

$$
\phi \Longrightarrow e^{-\frac{i}{2} \vec{\theta}(x) \cdot \vec{\tau}} \phi .
$$

In view of the potential minimum at $\phi^{\dagger} \phi=v=\frac{\mu}{\sqrt{\lambda}}$, one makes for the Higgs field the ansatz

$$
\phi(x)=e^{-\frac{i}{2 \sqrt{2} v} \vec{\tau} \cdot \vec{\xi}(x)}\left(\begin{array}{c}
0 \\
v+\frac{1}{\sqrt{2}} \eta(x)
\end{array}\right) .
$$


In the case of a spontaneously broken global symmetry, the $\vec{\xi}(x)$-field would describe massless Goldstone bosons. However, for a spontaneously broken local gauge symmetry, it can be transformed away by a proper gauge fixing. Then one has

$$
\phi(x)=\left(\begin{array}{c}
0 \\
v+\frac{1}{\sqrt{2}} \eta(x)
\end{array}\right),
$$

and the Lagrangian takes the form

$$
\mathcal{L}=-\frac{1}{4} \vec{F}_{\mu \nu} \cdot \vec{F}^{\mu \nu}+\frac{g^{2} v^{2}}{8} \vec{A}_{\mu} \cdot \vec{A}^{\mu}+\frac{1}{2} \partial^{\mu} \eta \partial_{\mu} \eta-\frac{\mu^{2}}{2} \eta^{2}+\cdots
$$

This Lagrangian now describes massive gauge bosons of mass $M_{B}=\frac{1}{2} g v$ as well as one massive Higgs particle which appears as consequence of the spontaneously broken local gauge symmetry.

\subsection{The Electroweak Lagrangian of the Standard Model}

To conclude this section, I will summarize the Lagrangian of the electroweak interaction of the standard model according to the Particle Data Group summary [PDG 96] (see also Chap. 6 of [Hua 92]). The standard model is based on the group $S U(2) \times U(1)$ with three gauge bosons $W_{\mu}^{j}(j=1,2,3)$ for $S U(2)$ and one gauge boson $B_{\mu}$ for $U(1)$. The corresponding gauge coupling constants are denoted by $g$ and $g^{\prime}$, respectively. The left-handed fermion fields (leptons and quarks)

$$
\psi_{L \alpha}=\left(\begin{array}{c}
\nu_{L \alpha} \\
l_{L \alpha}^{-}
\end{array}\right) \quad \text { and } \quad\left(\begin{array}{c}
u_{L \alpha} \\
d_{L \alpha}^{\prime}
\end{array}\right)
$$

of the $\alpha^{\text {th }}$ fermion family transform as doublets under $S U(2)$, where $d_{\alpha}^{\prime}=\sum_{\beta} V_{\alpha \beta} d_{\beta}(V$ is the Cabibbo-Kobayashi-Maskawa mixing matrix). The right-handed fermion fields $\psi_{R \alpha}$ transform as singlets under $S U(2)$. In the minimal model, one considers three lepton and quark fermion families and one complex Higgs doublet. Spontaneous symmetry breaking results in three massive gauge bosons, two charged ones $W^{ \pm}=\left(W^{1} \mp W^{2}\right) / \sqrt{2}$ and one neutral $Z=-B \sin \theta_{W}+W^{3} \cos \theta_{W}$, and one massless boson, the photon $A=B \cos \theta_{W}+W^{3} \sin \theta_{W}$, where $\theta_{W}=\tan ^{-1}\left(g^{\prime} / g\right)$ denotes the weak angle. The weak boson masses are given by $M_{W \pm}=80.22 \mathrm{GeV}$ and $M_{Z}=91.19 \mathrm{GeV}$.

After spontaneous symmetry breaking one arrives at the following fermion Lagrangian

$$
\begin{aligned}
\mathcal{L}_{F}= & \sum_{\alpha} \bar{\psi}_{\alpha}^{\dagger}\left(i \not \partial-m_{\alpha}-\frac{g m_{\alpha} H}{2 M_{W}}\right) \psi_{\alpha} \\
& -\frac{g}{2 \sqrt{2}} \sum_{\alpha} \bar{\psi}_{\alpha}^{\dagger} \gamma^{\mu}\left(1-\gamma^{5}\right)\left(T^{+} W_{\mu}^{+}+T^{-} W_{\mu}^{-}\right) \psi_{\alpha} \\
& -e \sum_{\alpha} Q_{\alpha} \bar{\psi}_{\alpha}^{\dagger} \gamma^{\mu} \psi_{\alpha} A_{\mu}-\frac{g}{2 \cos \theta_{W}} \sum_{\alpha} \bar{\psi}_{\alpha}^{\dagger} \gamma^{\mu}\left(g_{v}^{\alpha}-g_{a}^{\alpha} \gamma^{5}\right) \psi_{\alpha} Z_{\mu} .
\end{aligned}
$$

$T^{ \pm}$denote weak isospin raising and lowering operators, respectively. The elementary electric charge is given by $e=g \sin \theta_{W}$ and the vector and axial couplings are

$$
g_{v}^{\alpha}=t_{3}(\alpha)-2 Q_{\alpha} \sin ^{2} \theta_{W}, \quad g_{a}^{\alpha}=t_{3}(\alpha),
$$

where $t_{3}(\alpha)$ is the third component of the weak isospin of fermion $\alpha$ and $Q_{\alpha}$ its charge. The second term describes the charged-current weak interaction as, e.g., appears in beta decay $\left(G_{F} / \sqrt{2}=g^{2} / 8 M_{W}^{2}\right)$, the third term the e.m. interaction, and the last one the neutral-current weak interaction. 


\section{Properties of the Electromagnetic Operators}

In this Section I will give a brief review of the salient features of the e.m. current and two-photon operators which follow from the gauge invariance of the e.m. interaction. I will merely summarize the main results and refer the interested reader to my Schladming Lectures [Are 94], where I have discussed the gauge conditions in greater detail.

\subsection{Gauge Conditions for the Electromagnetic Operators}

In view of the fact, that the e.m. interaction is sufficiently weak, it is reasonable to assume that for the Hamiltonian of a hadronic system a Taylor expansion in the electromagnetic potential $A_{\mu}(x)$ exists, which reads up to second order

$$
\begin{aligned}
H_{e . m .}(A, t)= & \left.\int d^{3} x j_{\mu}(x) A^{\mu}(x)\right|_{x_{0}=t} \\
& +\left.\frac{1}{2} \int d^{3} x d^{3} y A^{\mu}(x) B_{\mu \nu}(x, y) A^{\nu}(y)\right|_{y_{0}=x_{0}=t}+\cdots,
\end{aligned}
$$

and which defines the e.m. current $j_{\mu}(x)$ and the two-photon operator $B_{\mu \nu}(x, y)$. Then gauge invariance leads to the following conditions for these operators:

(i) The continuity equation of the electromagnetic current

$$
\vec{\nabla} \cdot \vec{j}(x)+i\left[H_{0}, \rho(x)\right]+\partial_{t} \rho(x)=0,
$$

or covariantly written

$$
\partial_{\mu} j^{\mu}(x)=0
$$

which implies conservation of the total charge of the system.

(ii) The gauge condition for the two-photon operator

$$
\partial_{x}^{\mu} B_{\mu \nu}(x, y)=i\left[\rho(x), j_{\nu}(y)\right]
$$

which is essential for the derivation of the low energy theorem of the Compton amplitude.

\subsection{Low Energy Theorems}

An important consequence of these gauge conditions are the low-energy theorems, which allow to derive very simple relations for the current and two-photon operators in the limit that the photon momenta go to zero. In the case of the current, it leads to the famous Siegert theorem while for the photon scattering amplitude such a theorem has been derived by Sachs and Austern [SaA 51], and later on more general grounds by Low [Low 54] and Gell-Mann and Goldberger [GeG 54] for a spin-(1/2) particle.

To this end one first introduces the Fourier transforms of charge and current densities and the two-photon operator as well, which are here assumed to be time independent for convenience,

$$
\begin{aligned}
\tilde{\rho}(\vec{q}) & =\int d^{3} x \rho(\vec{x}) e^{i \vec{q} \cdot \vec{x}} \\
\vec{J}(\vec{q}) & =\int d^{3} x \vec{j}(\vec{x}) e^{i \vec{q} \cdot \vec{x}} \\
\tilde{B}_{k l}\left(\vec{q}^{\prime}, \vec{q}\right) & =\int d^{3} x d^{3} y e^{i \vec{q}^{\prime} \cdot \vec{x}} B_{k l}(\vec{x}, \vec{y}) e^{i \vec{q} \cdot \vec{y}} .
\end{aligned}
$$


Then the gauge condition for the current becomes

$$
\vec{q} \cdot \vec{J}(\vec{q})=\left[H_{0}, \tilde{\rho}(\vec{q})\right]
$$

Assuming that the operators possess a Taylor expansion with respect to $\vec{q}$, one finds up to first order

$$
J_{l}(\vec{q})=i\left[H_{0}, \vec{D}\right]-\frac{1}{2}\left[H_{0}, q_{l^{\prime}} Q_{l^{\prime} l}\right]-i(\vec{q} \times \vec{M})_{l}
$$

where $\vec{D}, \vec{M}$ and $Q_{l^{\prime} l}$ denote respectively, electric and magnetic dipole, and electric quadrupole operators. In particular, one has for $\vec{q}=0$

$$
J_{l}(0)=i\left[H_{0}, \vec{D}\right]
$$

which is called the Siegert theorem. It is very useful in cases, when the current density is less well known than the charge density, because it allows to evaluate the current from the knowledge of the charge density alone in the low energy regime via the charge dipole operator. A similar low energy expansion is found for the two-photon operator

$$
\begin{aligned}
\tilde{B}_{l k}\left(\vec{q}^{\prime}, \vec{q}\right)= & {\left[D_{k},\left[H_{0}, D_{l}\right]\right]+i\left[D_{k}, \frac{1}{2} q_{l^{\prime}}\left[H_{0}, Q_{l^{\prime} l}\right]-i(\vec{q} \times \vec{M})_{l}\right] } \\
& +i\left[D_{l}, \frac{1}{2} q_{l^{\prime}}^{\prime}\left[H_{0}, Q_{l^{\prime} k}\right]-i\left(\vec{q}^{\prime} \times \vec{M}\right)_{k}\right]
\end{aligned}
$$

yielding a corresponding Siegert theorem at $\vec{q}=\vec{q}^{\prime}=0$

$$
\tilde{B}_{l k}(0,0)=\left[D_{k},\left[H_{0}, D_{l}\right]\right] .
$$

\subsection{Multipole Decomposition}

As already mentioned, the observables are given in terms of the current matrix elements $J_{f i}^{\mu}$, which contain the information on the internal dynamics of the hadronic system. Since the intrinsic states of hadrons and nuclei can be classified according to the total angular momentum, it is very useful to decompose the charge and current operators in terms of multipole operators, which transfer a definite angular momentum. In this way one can take advantage of the angular momentum selection rules and thus can separate the geometrical aspects from the dynamical properties of the system. The other advantage of introducing these multipoles lies in the fact, that in principle, they can be determined from a complete set of observables and, thus, provide a convenient basis for the comparison between theory and experiment.

In detail, the multipole decomposition of charge and current density operators is given by

$$
J_{\lambda}(\vec{q})=(-)^{\lambda} \sqrt{2 \pi\left(1+\delta_{\lambda 0}\right)} \sum_{L M} i^{L} \hat{L} \mathcal{O}_{L M}^{\lambda} D_{M \lambda}^{L}\left(\phi_{q}, \theta_{q}, 0\right),
$$

with

$$
\mathcal{O}_{L M}^{\lambda}=\delta_{\lambda 0} C_{L M}+\delta_{|\lambda| 1}\left(T_{L M}^{(e)}+\lambda T_{L M}^{(m)}\right),
$$

with the charge multipole operators

$$
C_{L M}=\int d^{3} x \rho(\vec{x}) j_{L}(q x) Y_{L M}\left(\Omega_{x}\right),
$$


and the transverse electric and magnetic multipole operators, respectively,

$$
\begin{aligned}
T_{L M}^{(e)} & =\frac{1}{q} \int d^{3} x \vec{j}(\vec{x}) \cdot\left(\vec{\nabla} \times\left(j_{L}(q x) \vec{Y}_{L L M}\right)\right), \\
T_{L M}^{(m)} & =\int d^{3} x \vec{j}(\vec{x}) \cdot\left(j_{L}(q x) \vec{Y}_{L L M}\right) .
\end{aligned}
$$

Here, the vector spherical harmonics are defined by

$$
\vec{Y}_{L \ell M}=\sum_{m \lambda}(\ell m 1 \lambda \mid L M) Y_{\ell m} \vec{e}_{\lambda}
$$

Since the multipole operators are irreducible spherical tensors of rank $L$, one can make use of the Wigner-Eckart theorem in evaluating them between states of definite angular momentum $|J M\rangle$ :

$$
\begin{aligned}
\left\langle J_{f} M_{f}\left|J_{\lambda}(\vec{q})\right| J_{i} M_{i}\right\rangle= & (-)^{\lambda+J_{f}-M_{f}} \sqrt{2 \pi\left(1+\delta_{\lambda 0}\right)} \sum_{L M} i^{L} \hat{L}\left(\begin{array}{ccc}
J_{f} & L & J_{i} \\
-M_{f} & M & M_{i}
\end{array}\right) \\
& \left\langle J_{f}\left\|\mathcal{O}_{L}^{\lambda}\right\| J_{i}\right\rangle D_{M \lambda}^{L}\left(\phi_{q}, \theta_{q}, 0\right) .
\end{aligned}
$$

The $3 j$-symbol contains the geometrical aspects, i.e., angular momentum selection rules, while the multipole strength is given by the reduced matrix element $\left\langle J_{f}\left\|\mathcal{O}_{L}^{\lambda}\right\| J_{i}\right\rangle$, which contain the information on the internal dynamics of the system.

\subsection{Generalized Siegert Theorem and Siegert Operators}

The above mentioned Siegert theorem can be generalized to the statement that in the low energy limit the transverse electric multipoles can be related to the charge multipoles of the same order. This very important theorem for photo- and electronuclear theory allows then to calculate reliably within the limits of the theorem the electric transition matrix elements from the charge density without explicit knowledge of the currents, e.g., exchange currents.

The essential idea (for details see [Are 94]) rests on the observation that the transverse electric multipole field $\vec{A}_{L M}(e)$ can be decomposed into a gradient field and a term which is two orders higher in $q x$

$$
\vec{A}_{L M}(e)=\vec{\nabla} \Phi_{L M}(\vec{x}, q)+\vec{A}_{L M}^{\prime}(e ; \Phi)
$$

where the specific form of ${\overrightarrow{A_{L M}^{\prime}}}^{\prime}(e ; \Phi)$ depends on the choice of $\Phi_{L M}$ [ScW 90].

With the help of current conservation, one arrives at

$$
\begin{aligned}
\left\langle f\left|T_{L M}^{(e)}\right| i\right\rangle= & i \int d^{3} x\left\langle f\left|\left[H^{i n t}, \rho(\vec{x})\right]\right| i\right\rangle \Phi_{L M}(\vec{x}, q) \\
& +\int d^{3} x\left\langle f\left|\vec{j}_{\text {int }}(\vec{x})\right| i\right\rangle \cdot \vec{A}_{L M}^{\prime}(e ; \Phi),
\end{aligned}
$$

where the first term defines a Siegert operator for the electric multipole whose form depends on the choice of $\Phi_{L M}$. The sub- and superscript "int" refers to the internal motion [ScW 90]. A particularly important consequence is that a dominant part of meson exchange currents essentially in the electric dipole $(E 1)$ - is already included implicitly in the Siegert operators [Are 81a, GaH 81]. 


\section{Models for the Electromagnetic Operators}

From the fermion part of the Lagrangian of the standard model in (2.35) one obtains immediately the electromagnetic and neutral currents from the coupling of the fermions to photon and $Z$-boson, respectively, by writing these couplings in the form

$$
\mathcal{L}_{F}^{\gamma}+\mathcal{L}_{F}^{Z}=-e j_{\mu}^{(\gamma)} A^{\mu}-\frac{g}{2 \cos \theta_{W}}\left(j_{\mu}^{\left(Z_{v}\right)}-j_{\mu}^{\left(Z_{a}\right)}\right) Z^{\mu}
$$

where one has for the e.m. current

$$
j_{\mu}^{(\gamma)}=\sum_{\alpha} Q_{\alpha} \bar{\psi}_{\alpha}^{\dagger} \gamma_{\mu} \psi_{\alpha}
$$

The neutral current splits into a vector

$$
j_{\mu}^{\left(Z_{v}\right)}=\sum_{\alpha} g_{v}^{\alpha} \bar{\psi}_{\alpha}^{\dagger} \gamma_{\mu} \psi_{\alpha}
$$

and an axial vector current

$$
j_{\mu}^{\left(Z_{a}\right)}=\sum_{\alpha} g_{a}^{\alpha} \bar{\psi}_{\alpha}^{\dagger} \gamma_{\mu} \gamma_{5} \psi_{\alpha}
$$

Here, I will not consider the additional charged currents describing the coupling to the charged bosons $W^{ \pm}$, which are also given in (2.35).

In principle, in order to get the hadronic currents, one has to evaluate these operators for the quark fields between the internal hadron wave functions given in terms of quark and gluon d.o.f. Unfortunately, these are not known yet due to the difficulties one encounters in solving QCD in the non-perturbative regime. Therefore one resorts either to effective hadron models, e.g., constituent quark models, or to a purely phenomenological description. In the latter case, one makes the most general ansatz for the one-body hadron currents which is allowed by Lorentz covariance, parity and time reversal invariance. The remaining form factors, undetermined functions of the only independent Lorentz scalar $q^{2}$ for on-shell particles, have to be taken from experiment. I will now briefly review the one- and two-body electroweak currents for nucleons.

\subsection{One-Body Currents}

The on-shell nucleon vector currents (e.m. and neutral), fulfilling the requirements of Lorentz covariance, parity and time reversal invariance, have the well known form

$$
\left\langle p^{\prime}\left|J_{\mu}^{V}(0)\right| p\right\rangle=\bar{u}\left(p^{\prime}\right)\left[F_{1}\left(q^{2}\right) \gamma_{\mu}+\frac{i}{2 M} F_{2}\left(q^{2}\right) \sigma_{\mu \nu} q^{\nu}\right] u(p)
$$

with $q=p^{\prime}-p$ and the Dirac and Pauli form factors, $F_{1}\left(q^{2}\right)$ and $F_{2}\left(q^{2}\right)$, respectively. The nucleon mass is denoted by $M$. For off-shell nucleons additional form factors arise which, however, are model dependent and not directly observable. It is convenient to introduce the Sachs form factors by

$$
\begin{aligned}
G_{E}\left(q^{2}\right) & =F_{1}\left(q^{2}\right)+\tau F_{2}\left(q^{2}\right), \quad \text { where } \tau=\frac{q^{2}}{4 M^{2}}, \\
G_{M}\left(q^{2}\right) & =F_{1}\left(q^{2}\right)+F_{2}\left(q^{2}\right),
\end{aligned}
$$

because these are directly observable, for example, in elastic electron nucleon scattering for the e.m. form factors. The simplest models for them are the purely phenomenological dipole 
form factors [GaK 71] or the ones of the vector meson dominance model (VMD) [HoP 76]. Applying a $p / M$-expansion, one finds the corresponding nonrelativistic expressions

$$
\begin{aligned}
\left\langle p^{\prime}|\rho(0)| p\right\rangle & =\chi^{\dagger} G_{E}\left(q^{2}\right) \chi, \\
\left\langle p^{\prime}|\vec{j}(0)| p\right\rangle & =\chi^{\dagger} \frac{1}{2 M}\left[G_{E}\left(q^{2}\right)\left(\vec{p}^{\prime}+\vec{p}\right)+i G_{M}\left(q^{2}\right) \vec{\sigma} \times \vec{q}\right] \chi,
\end{aligned}
$$

where $\chi^{\prime}$ and $\chi$ denote the nucleon Pauli spinors. Note, that the current density is one order in $p / M$ higher than the charge density.

Correspondingly, one finds for the axial currents for on-shell nucleons the general form

$$
\left\langle p^{\prime}\left|J_{\mu}^{A}(0)\right| p\right\rangle=\bar{u}\left(p^{\prime}\right)\left[G_{A}\left(q^{2}\right) \gamma_{\mu}+\frac{G_{P}\left(q^{2}\right)}{M} q_{\mu}\right] \gamma_{5} u(p),
$$

with the axial form factor $G_{A}\left(q^{2}\right)$ and the induced pseudoscalar form factor $G_{P}\left(q^{2}\right)$. Again, the nonrelativistic reduction reads



$$
\begin{aligned}
& \left\langle p^{\prime}\left|\vec{j}^{A}(0)\right| p\right\rangle=-\chi^{\prime \dagger}\left[G_{A}\left(q^{2}\right) \vec{\sigma}\right] \chi .
\end{aligned}
$$

Here, it is the axial charge density which is one order in $p / M$ higher than the current density. A discussion of the neutral current form factors (vector and axial) and their phenomenological parametrizations is given in $[\mathrm{MuD} 94]$.

\subsection{Two-Body Meson Exchange Currents}

I can be very brief in this case, because this topic will be covered by the lectures of J.F. Mathiot at this workshop [Mat 97]. I only will give as most important example the explicit form of the pion exchange current in lowest, i.e., static order

$$
\begin{aligned}
\rho_{[2]}^{\pi}(\vec{x})= & 0, \\
\vec{j}_{[2]}^{\pi}(\vec{x})= & -\frac{f_{\pi}^{2}}{m_{\pi}^{2}}\left(\vec{\tau}_{1} \times \vec{\tau}_{2}\right)_{3}\left[\delta\left(\vec{x}-\vec{r}_{1}\right) \vec{\sigma}_{1}\left(\vec{\sigma}_{2} \cdot \vec{\nabla}_{2}\right) J_{m_{\pi}}\left(r_{12}\right)-(1 \leftrightarrow 2)\right. \\
& \left.+\left(\vec{\sigma}_{1} \cdot \vec{\nabla}_{1}\right)\left(\vec{\sigma}_{2} \cdot \vec{\nabla}_{2}\right) J_{m_{\pi}}\left(\vec{r}_{1}-\vec{x}\right) \overleftrightarrow{\nabla}_{x} J_{m_{\pi}}\left(\vec{x}-\vec{r}_{2}\right)\right],
\end{aligned}
$$

with the Yukawa function

$$
J_{m_{\pi}}(r)=\frac{e^{-m_{\pi} r}}{4 \pi r}
$$

Pion mass and $\pi N$ coupling constant are denoted by $m_{\pi}$ and $f_{\pi}$, respectively. The vanishing of the charge density supports the early hypothesis of Siegert [Sie 37], expressing the fact that the charge densities of the oppositely charged mesons, exchanged in opposite directions between proton and neutron and contributing with equal weight, cancel each other, whereas the currents associated with them do add. The first line of (4.14) describe the pair or contact current, and the second the pion current, where the photon interacts with the pion in flight (see Fig. 4.1 for a diagrammatic representation). Analogous contributions arise from heavier meson exchanges $(\sigma, \rho, \omega \ldots)$ and also $\rho / \omega \pi \gamma$ [AdT 89, AdA 97]. Furthermore, similar twobody contributions exist for the neutral currents [HaH 89].

Also for the two-photon operator, one will have corresponding exchange contributions. I show in Fig. 4.2 as an example the lowest order $\pi$ exchange contribution to the two-photon operator [Fri 76, Are 80]. 




Fig. 4.1 Pair or contact and pion current contributions to the pion exchange current.

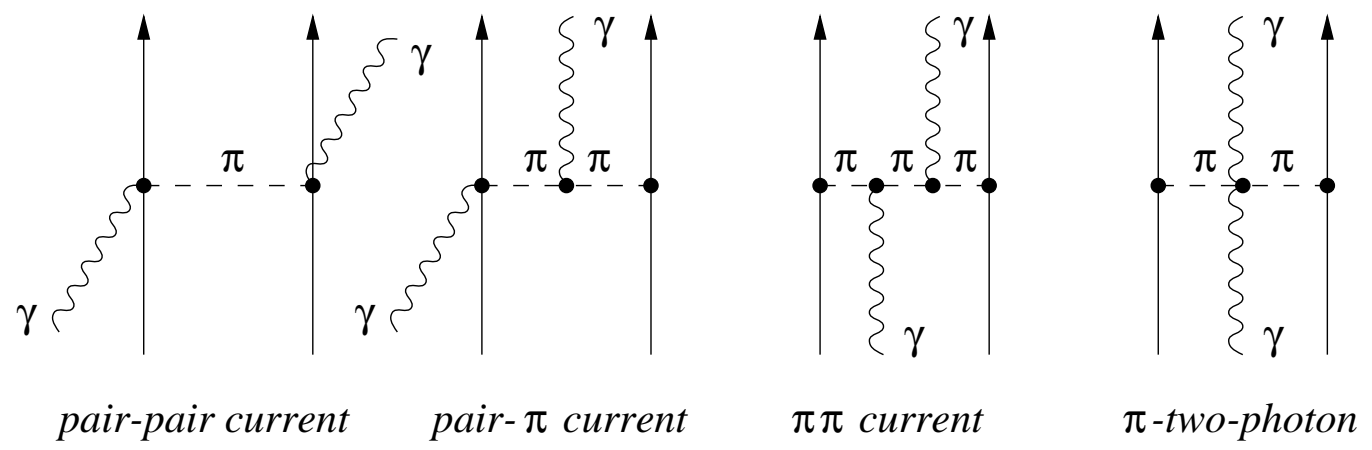

Fig. 4.2 Various pair and pion current contributions to the pion exchange two-photon operator.

\subsection{Isobar Currents}

The existence of internal nucleon structure as manifest in the rich spectrum of internally excited nucleon resonances, or nucleon isobars, leads naturally to additional e.m. interaction operators in terms of nucleon-isobar transition currents as well as diagonal isobar currents. As most prominent example let me give the nonrelativistic expressions for the $\Delta(1232)$ resonance (for details see [WeA 78]).

(i) $N \Delta$ transition current (nonrelativistic):

$$
\begin{aligned}
& \left\langle\Delta p^{\prime}|\rho(0)| N p\right\rangle=0, \\
& \left\langle\Delta p^{\prime}|\vec{j}(0)| N p\right\rangle=\frac{1}{2 M} \chi_{\Delta}^{\prime \dagger}\left[G_{M}\left(q^{2}\right) \vec{\sigma}_{\Delta N} \times \vec{q}\right] \chi,
\end{aligned}
$$

where $\chi_{\Delta}^{\prime}$ denotes the final $\Delta$-(3/2)-spinor and $\sigma_{\Delta N}$ the $N \rightarrow \Delta$ transition spin operator. Here I have restricted myself to the dominant $M 1$ part neglecting the small $C 2$ and $E 2$ contributions.

(ii) diagonal $\Delta$ current (nonrelativistic):

$$
\begin{aligned}
\left\langle\Delta p^{\prime}|\rho(0)| N p\right\rangle & =\chi_{\Delta}^{\prime \dagger} G_{E}^{\Delta}\left(q^{2}\right) \chi_{\Delta}, \\
\left\langle\Delta p^{\prime}|\vec{j}(0)| N p\right\rangle & =\frac{1}{2 M_{\Delta}} \chi_{\Delta}^{\prime \dagger}\left[G_{E}^{\Delta}\left(q^{2}\right)\left(\vec{p}^{\prime}+\vec{p}\right)+i G_{M}^{\Delta}\left(q^{2}\right) \vec{\sigma}_{\Delta} \times \vec{q}\right] \chi_{\Delta},
\end{aligned}
$$

where $M_{\Delta}$ denotes the $\Delta$ mass. The electric and magnetic form factors of the $\Delta$ are denoted by $G_{E}^{\Delta}\left(q^{2}\right)$ and $G_{M}^{\Delta}\left(q^{2}\right)$, respectively. Possible $E 2$ and $M 3$ contributions have been neglected for convenience. Analogous currents appear for higher resonances $\left(N(1440), D_{13}(1520), S_{11}(1535), \ldots\right)$ [WeA 78, ScW 96]. 




Fig. 4.3 Isobar contributions to the e.m. current as effective two-body operators.

These isobar currents can be incorporated either in terms of effective, non-local two-body operators where the intermediate appearance of an isobar is implicitly included (see Fig. 4.3) or in the framework of nuclear isobar configurations (IC) as explicit constituents of the nuclear wave function [ArW 72, Gre 76, WeA 78].

\subsection{Meson Production Currents}

The internal nucleon structure becomes also manifest in the e.m. meson production on a nucleon. As an example, I will consider briefly pion photoproduction. More detailed reviews may be found in [Dav 94]. The lowest order tree diagrams for the elementary production process on the nucleon are shown in Figs. 4.4 and 4.5 for the resonance contribution and the Born terms. Restriction to these lowest order tree diagrams would violate unitarity. Therefore, the tree diagrams have to be either unitarized [Dav 94] or used as driving terms in a dynamical model as represented diagrammatical in Fig. 4.6. A recent critical discussion of the various methods is given in [WiW 98], where it is emphasized, that the resonance excitation multipoles cannot be extracted in a model independent way, and thus do not constitute observables in the strict sense [WiW 96] (see also the seminar by Th. Wilbois at this workshop).
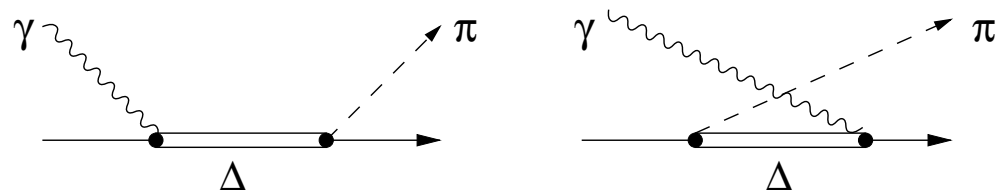

Fig. 4.4 Direct and crossed $\Delta$ resonance contributions to pion photoproduction.

\subsection{Relativistic Contributions}

As last topic in this section, I will briefly touch upon the role of relativity. It is clear that with increasing energy and momentum transfers, relativistic effects will become significant and, thus, have to be included. One may distinguish different types of relativistic contributions. First of all, there are relativistic contributions to the electroweak operators. For example, from the relativistic expression in (4.5) on obtains in the $p / M$-expansion as leading order relativistic contribution the spin-orbit and Darwin-Foldy currents plus additional terms. Analogous contributions arise for the two-body currents, in particular from retardation in propagators of the exchanged mesons. Secondly, relativistic effects appear in the 


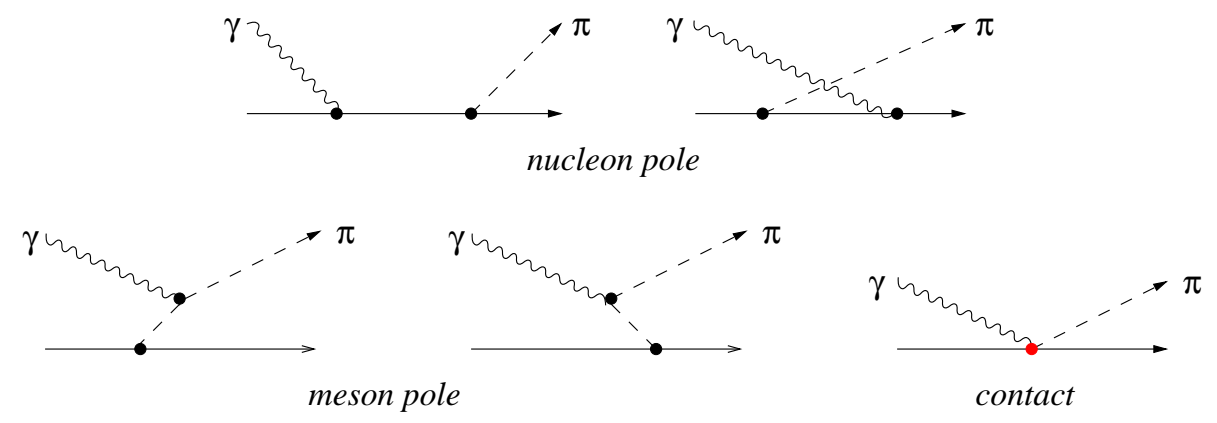

Fig. 4.5 Born contributions to pion photoproduction.

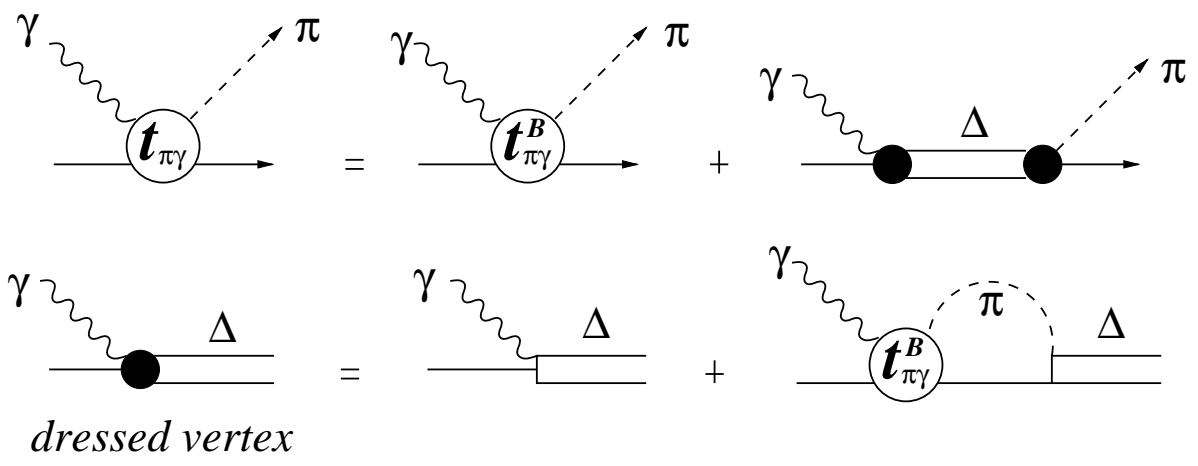

Fig. 4.6 Dynamical model of pion photoproduction.

internal rest-frame wave function from the internal relativistic dynamics of the hadrons. Additional effects arise from the Lorentz boost of the internal wave function from the rest system to a moving frame taking into account the effect of Lorentz contraction and Thomas-Wigner rotation. Since the Lorentz boost is represented by a unitary operator acting on the rest frame wave function, its effect can be incorporated into the current density operators so that these are evaluated between rest frame wave functions. A large part of these operators is determined from the fact, that they have to fulfil the requirements of Lorentz covariance which is formulated in the form of commutator relations with the generators of the Poincaré group. A systematic exploitation of these relations can be found in [AdA 96], and a complete listing of all leading order one- and two-body currents for scalar, vector and pseudoscalar meson exchange is given in [AdA 97], which incorporate also the boost contributions.

\section{Basic Electroweak Processes}

Because of the small electroweak coupling constants, the most important processes are the one- and two-boson processes, whereby free one- and two-boson processes are only realizable with photons.

\subsection{One-Boson Processes}

It is useful to distinguish between real and virtual one-boson processes:

(i) Real photon reactions comprise absorption and emission by a hadronic system with internal excitation or deexcitation, respectively (see Fig. 5.1a). Since real photons have transverse polarization, only the transverse current contributes. Energy and momentum transfers are not independent from each other but are related by $\omega=q$. 


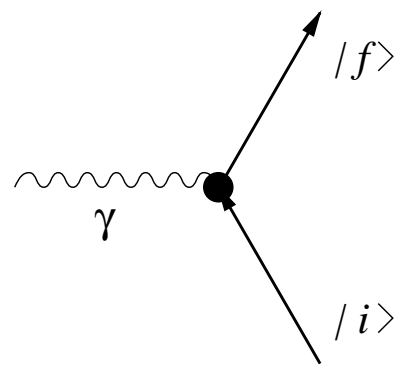

a)

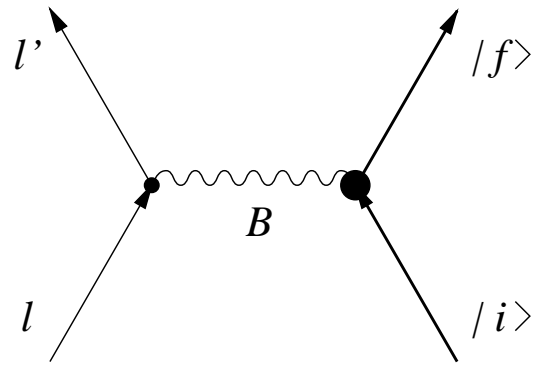

b)

Fig. 5.1 a) Photon absorption or emission by a hadronic system; b) One-boson exchange diagram for lepton scattering off a hadron.

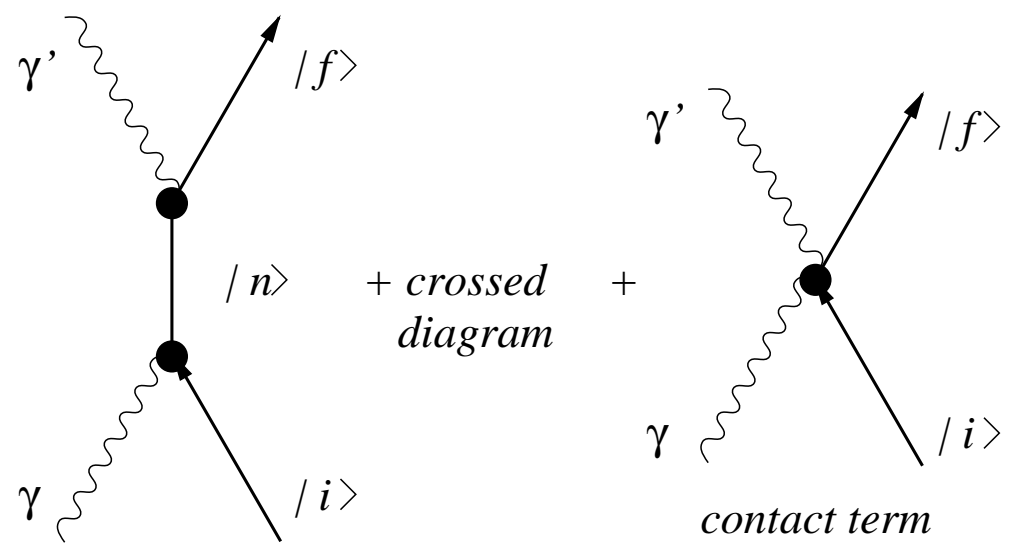

Fig. 5.2 Two-step or dispersion (left) and one-step or contact (right) contributions to photon scattering.

(ii) Virtual bosons appear in lepton scattering off a hadron, being exchanged between the lepton and the target as, for example, in elastic and inelastic electron and neutrino scattering off hadronic systems (see Fig. 5.1b). Energy and momentum transfers can be varied independently within the range $\omega^{2}<q^{2}$, and in addition also longitudinal polarization of the virtual boson will contribute. For $\left(e, e^{\prime}\right)$ and $\left(\nu, \nu^{\prime}\right)$ one has a neutral current interaction $(B=\gamma, Z)$, while a charged current interaction $\left(B=W^{ \pm}\right)$appears in $(e, \nu)$, for example. Since the weak gauge bosons couple to both, vector and axial vector currents, one has the interesting phenomenon of parity violation, e.g. in $\left(e, e^{\prime}\right)$, which arises via the interference

of $\gamma$ - and $Z$-exchange. Indeed, at present several experiments are underway to determine the strange sea quark contribution to the nucleon form factors from the parity violating asymmetry of longitudinally polarized electrons (see Sect. 6.4).

\subsection{Two-Boson Processes}

In the case of two-boson processes, a variety reactions are possible depending on the participation of either two real photons, one real photon and one virtual boson, or two virtual bosons. I have selected for illustration a few examples:

(i) Fig. 5.2 shows the lowest order diagrams contributing to elastic and inelastic photon scattering (Compton and Raman scattering). I would like to emphasize, that the separation into two-step (dispersion diagram) and one-step (contact diagram) contributions is gauge dependent, which means that the splitting into these two contributions is not observable. 
(ii) A bremsstrahlung process with one virtual boson exchange and a real photon emission on the hadronic side is depicted on the left side of Fig. 5.3. Here, the exchanged virtual boson can be both, a $\gamma$ or a $Z$. A corresponding process can occur on the leptonic side.

(iii) Finally, a dispersion contribution to electron scattering as a second order correction to the leading one-photon exchange contribution is shown on the right side of Fig. 5.3. It corresponds to the Compton process with two virtual photons. Also here, $Z$-exchange is possible in both places.

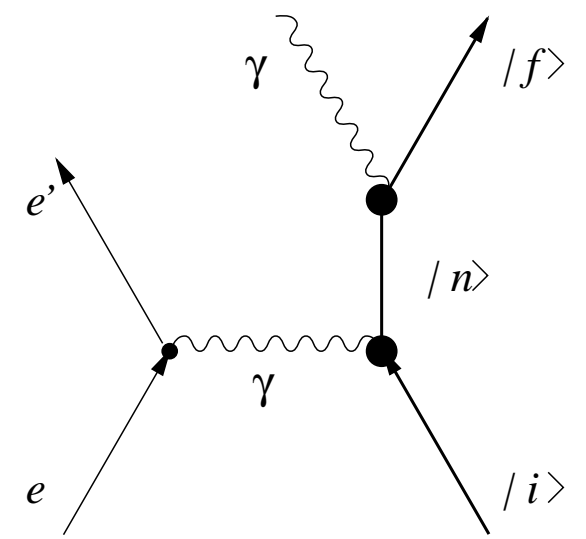

a)

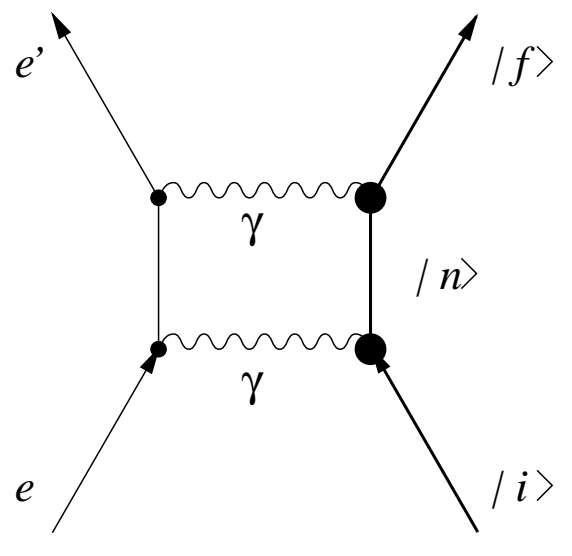

b)

Fig. 5.3 Left: Bremsstrahlungs process at the hadronic leg; Right: Dispersion contribution to electron scattering.

\subsection{Cross Section for Electron Scattering}

Electron scattering is a particularly important example of a one-photon process. Evaluation of the diagram in Fig. 5.4 leads to the well-known expression for the cross section

$$
d \sigma_{f i}=(2 \pi)^{-5} \delta^{(4)}\left(P_{f}-q-P_{i}\right) \frac{m_{e}^{2} d^{3} k_{f}}{4 k_{i 0} k_{f 0} M_{i}} \sum_{f} \operatorname{tr}\left(\mathcal{M}_{f i}^{\dagger} \hat{\rho}^{f} \mathcal{M}_{f i} \hat{\rho}^{e} \hat{\rho}^{i}\right),
$$

where $q=k_{i}-k_{f}$ denotes the momentum transfer. The notation for the momenta of electron and hadron is explained in Fig. 5.4. The initial electron and hadron polarization density matrices are denoted by $\hat{\rho}^{e}$ and $\hat{\rho}^{i}$, respectively. The phase space density of the final states including possible polarization analysis is described by $\hat{\rho}^{f}$. Its specific form depends on the

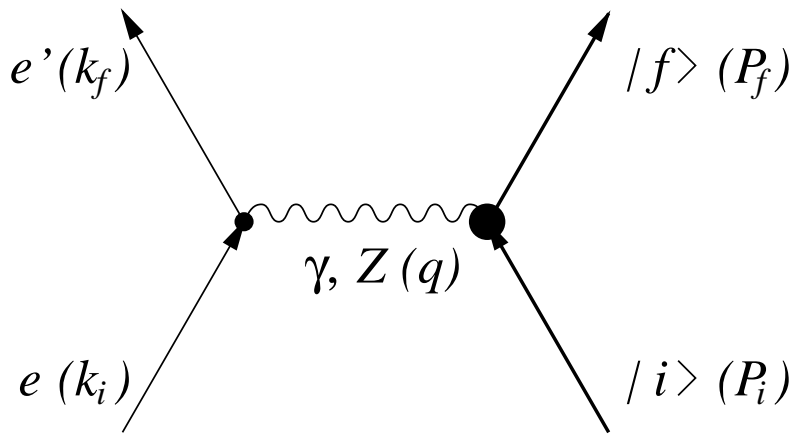

Fig. 5.4 One-photon exchange diagram for electron scattering. 
experimental conditions, whether one considers an inclusive process with no specific analysis of the final states, or an exclusive reaction with a more detailed analysis of the final hadronic system.

In the one-boson-exchange approximation, the invariant matrix element $\mathcal{M}_{f i}$ contains two contributions from virtual $\gamma$ and $Z$ exchange with the latter naturally being strongly suppressed at not too high momentum transfers $\left(-q_{\mu}^{2} \ll M_{Z}^{2}\right)$,

$$
\mathcal{M}_{f i}=\frac{e^{2}}{q_{\mu}^{2}} j^{(\gamma) \mu} J_{f i, \mu}^{(\gamma)}+\sqrt{2} G_{F} j^{(Z) \mu} J_{f i, \mu}^{(Z)} .
$$

The lepton and hadron currents are denoted by $j_{\mu}^{(\gamma / Z)}$ and $J_{f i, \mu}^{(\gamma / Z)}$, respectively. The superscripts " $\gamma$ " and " $Z$ " indicate the electromagnetic and weak neutral current contributions. Allowing for longitudinal electron polarization of degree $h$, one finds

$$
\begin{aligned}
m_{e}^{2} \delta^{(4)}\left(P_{f}-q-P_{i}\right) \sum_{f} & \operatorname{tr}\left(\mathcal{M}_{f i}^{\dagger} \hat{\rho}^{f} \mathcal{M}_{f i} \hat{\rho}^{e} \hat{\rho}^{i}\right) \\
= & \left(\frac{e^{2}}{q_{\mu}^{2}}\right)^{2} \eta_{\mu \nu}^{(\gamma \gamma)}(h) W_{f i}^{(\gamma \gamma) \mu \nu}+2 G_{F}^{2} \eta_{\mu \nu}^{(Z Z)}(h) W_{f i}^{(Z Z) \mu \nu} \\
& +\sqrt{2} G_{F} \frac{e^{2}}{q_{\mu}^{2}} \eta_{\mu \nu}^{(\gamma Z)}(h)\left(W_{f i}^{(\gamma Z) \mu \nu}+W_{f i}^{(Z \gamma) \mu \nu}\right) .
\end{aligned}
$$

Here, the various lepton tensors are given by

$$
\begin{aligned}
\eta_{\mu \nu}^{(\gamma \gamma)}(h) & =\eta_{\mu \nu}^{v v}(h) \\
\eta_{\mu \nu}^{(\gamma Z)}(h) & =g_{V} \eta_{\mu \nu}^{v v}(h)+g_{A} \eta_{\mu \nu}^{v a}(h), \\
\eta_{\mu \nu}^{(Z Z)}(h) & =\left(g_{V}^{2}+g_{A}^{2}\right) \eta_{\mu \nu}^{v v}(h)+2 g_{V} g_{A} \eta_{\mu \nu}^{v a}(h) .
\end{aligned}
$$

Since in contrast to the $\gamma$, the $Z$ couples to both vector and axial vector current, the additional tensor $\eta_{\mu \nu}^{v a}$ arises in $\eta_{\mu \nu}^{(\gamma Z)}$ and $\eta_{\mu \nu}^{(Z Z)}$, whereas the pure electromagnetic case is described by the tensor $\eta_{\mu \nu}^{v v}$ alone. Both tensors can be parametrized as

$$
\begin{aligned}
& \eta_{\mu \nu}^{v v}(h)=\eta_{\mu \nu}^{0}+h \eta_{\mu \nu}^{\prime}, \\
& \eta_{\mu \nu}^{v a}(h)=\eta_{\mu \nu}^{\prime}+h \eta_{\mu \nu}^{0},
\end{aligned}
$$

where in the high energy limit, i.e., electron mass $m_{e} \rightarrow 0$, the tensors $\eta_{\mu \nu}^{0}$ and $\eta_{\mu \nu}^{\prime}$ have the explicit form

$$
\begin{aligned}
\eta_{\mu \nu}^{0} & =\left(k_{i \mu} k_{f \nu}+k_{f \mu} k_{i \nu}\right)-g_{\mu \nu} k_{i} \cdot k_{f} \\
& =\frac{1}{2}\left(k_{\mu} k_{\nu}-q_{\mu} q_{\nu}+g_{\mu \nu} q_{\rho}^{2}\right), \\
\eta_{\mu \nu}^{\prime} & =i \varepsilon_{\mu \nu \alpha \beta} k_{i}^{\alpha} k_{f}^{\beta} \\
& =\frac{i}{2} \varepsilon_{\mu \nu \alpha \beta} k^{\alpha} q^{\beta},
\end{aligned}
$$

where $k=k_{i}+k_{f}$. The hadronic tensors are given by the electromagnetic and weak current matrix elements

$$
W_{f i}^{\left(B B^{\prime}\right) \mu \nu}=\sum_{f} \operatorname{tr}\left(J_{f i}^{(B) \mu \dagger} \hat{\rho}^{f} J_{f i}^{\left(B^{\prime}\right) \nu} \hat{\rho}^{i}\right) \delta^{(4)}\left(P_{f}-P_{i}-q\right)
$$

where $B, B^{\prime} \in\{\gamma, Z\}$, and the trace refers to the spin quantum numbers of the hadronic initial state. 
Proceeding now as in the pure electromagnetic case, one obtains for the differential cross section including both beam and target polarization

$$
\begin{aligned}
\frac{d^{3} \sigma^{\gamma+Z}}{d k_{f}^{l a b} d \Omega_{k_{f}}^{l a b}}= & \frac{\alpha}{2 \pi^{2}} \frac{k_{f}^{l a b}}{k_{i}^{l a b} q_{\mu}^{4}} \sum_{\lambda \lambda^{\prime}=1}^{3} \sum_{m_{i} m_{i}^{\prime}} \rho_{m_{i} m_{i}^{\prime}}^{i}\left(\rho_{\lambda \lambda^{\prime}}^{v v} \sum_{f} J_{f \lambda^{\prime} m_{i}^{\prime}}^{(\gamma) *} \hat{\rho}^{f} J_{f \lambda m_{i}}^{(\gamma)}\right. \\
& \left.+\frac{G_{F}}{\sqrt{2}} \frac{q_{\mu}^{2}}{\pi \alpha}\left(g_{V} \rho_{\lambda \lambda^{\prime}}^{v v}+g_{A} \rho_{\lambda \lambda^{\prime}}^{v a}\right) \Re e\left[\sum_{f} J_{f \lambda^{\prime} m_{i}^{\prime}}^{(Z) *} \hat{\rho}^{f} J_{f \lambda m_{i}}^{(\gamma)}\right]\right) .
\end{aligned}
$$

The terms quadratic in the weak amplitude $\left(J_{\mu}^{(Z) *} J_{\nu}^{(Z)}\right)$ have been omitted since they are of order $\mathcal{O}\left(q_{\mu}^{4} / M_{Z}^{4}\right)$ compared to the electromagnetic process. The index $\lambda$ of the hadronic current matrix elements $J_{f \lambda m_{i}}^{(\gamma / Z)}$ refers for $\lambda= \pm 1$ to the transverse current components (with respect to $\vec{q}$ ), while for $\lambda=0$ the component is given by a linear combination of charge and longitudinal current

$$
J_{0}=-\frac{|\vec{q}|^{2}}{q_{\mu}^{2}}\left(\rho-\frac{\omega}{|\vec{q}|^{2}} \vec{q} \cdot \vec{J}\right)=\rho-\frac{\omega}{q_{\mu}^{2}}(\omega \rho-\vec{q} \cdot \vec{J}),
$$

which reduces to the charge density $\rho$ for a conserved current.

The spherical components of the two types of virtual boson density matrices are

$$
\begin{aligned}
& \rho_{\lambda \lambda^{\prime}}^{v v}=\rho_{\lambda \lambda^{\prime}}^{0}+h \rho_{\lambda \lambda^{\prime}}^{\prime}, \\
& \rho_{\lambda \lambda^{\prime}}^{v a}=\rho_{\lambda \lambda^{\prime}}^{\prime}+h \rho_{\lambda \lambda^{\prime}}^{0},
\end{aligned}
$$

and they obey the symmetry relations

$$
\begin{aligned}
\rho_{\lambda \lambda^{\prime}}^{v v / v a} & =\rho_{\lambda^{\prime} \lambda}^{v v / v a}, \\
\rho_{-\lambda-\lambda^{\prime}}^{0} & =(-)^{\lambda+\lambda^{\prime}} \rho_{\lambda \lambda^{\prime}}^{0}, \\
\rho_{-\lambda-\lambda^{\prime}}^{\prime} & =(-)^{\lambda+\lambda^{\prime}+1} \rho_{\lambda \lambda^{\prime}}^{\prime} .
\end{aligned}
$$

The nonvanishing components for unpolarized electrons are (note $q^{2}<0$ )

$$
\begin{aligned}
\rho_{L}=\rho_{00}^{0}=-\beta^{2} q_{\nu}^{2} \frac{\xi^{2}}{2 \eta}, & \rho_{T}=\rho_{11}^{0}=-\frac{1}{2} q_{\nu}^{2}\left(1+\frac{\xi}{2 \eta}\right), \\
\rho_{L T}=\rho_{01}^{0}=-\beta q_{\nu}^{2} \frac{\xi}{\eta} \sqrt{\frac{\eta+\xi}{8}}, & \rho_{T T}=\rho_{-11}^{0}=q_{\nu}^{2} \frac{\xi}{4 \eta}
\end{aligned}
$$

and for longitudinally polarized electrons

$$
\rho_{L T}^{\prime}=\rho_{01}^{\prime}=-\frac{1}{2} \beta \frac{q_{\nu}^{2}}{\sqrt{2 \eta}} \xi, \quad \rho_{T}^{\prime}=\rho_{11}^{\prime}=-\frac{1}{2} q_{\nu}^{2} \sqrt{\frac{\eta+\xi}{\eta}}
$$

with

$$
\beta=\frac{\left|\vec{q}^{l a b}\right|}{\left|\vec{q}^{c}\right|}, \quad \xi=-\frac{q_{\nu}^{2}}{\left|\vec{q}^{l a b}\right|^{2}}, \quad \eta=\tan ^{2} \frac{\theta_{e}}{2}
$$

where $\beta$ expresses the boost from the lab system to the frame in which the hadronic tensor is evaluated and $\vec{q}^{c}$ denotes the momentum transfer in this frame. The relation of the kinematic functions $\rho_{\alpha}^{(\prime)}$ to the kinematic functions $v_{\alpha^{(\prime)}}$ of [MuD 94] are simply given by

$$
\rho_{\alpha}^{(\prime)}=-\frac{q_{\mu}^{2}}{2 \eta} v_{\alpha^{(\prime)}}
$$


where $\alpha \in\{L, T, L T, T T\}$.

The final result for the cross section for an unpolarized target is then

$$
\begin{aligned}
\frac{d^{3} \sigma^{\gamma+Z}}{d k_{f}^{l a b} d \Omega_{k_{f}}^{l a b}}= & c\left\{\rho_{L}\left(W_{L}^{\gamma_{v}}+a_{v} W_{L}^{Z_{v}}\right)+\rho_{T}\left(W_{T}^{\gamma_{v}}+a_{v} W_{T}^{Z_{v}}\right)\right. \\
& +\rho_{L T}\left(\left(W_{L T}^{\gamma_{v}}+a_{v} W_{L T}^{Z_{v}}\right)+\left(W_{L T}^{\gamma_{a}}+a_{v} W_{L T}^{Z_{a}}\right)\right) \\
& +\rho_{T T}\left(\left(W_{T T}^{\gamma_{v}}+a_{v} W_{T T}^{Z_{v}}\right)+\left(W_{T T}^{\gamma_{a}}+a_{v} W_{T T}^{Z_{a}}\right)\right) \\
& -\rho_{T}^{\prime} a_{a} W_{T}^{\prime Z_{a}}-\rho_{L T}^{\prime} a_{a}\left(W_{L T}^{\prime Z_{a}}-W_{L T}^{\prime Z_{v}}\right) \\
& +h\left[\rho_{L} a_{a} W_{L}^{Z_{v}}+\rho_{T} a_{a} W_{T}^{Z_{v}}+\rho_{L T} a_{a}\left(W_{L T}^{Z_{v}}+W_{L T}^{Z_{a}}\right)\right. \\
& +\rho_{T T} a_{a}\left(W_{T T}^{Z_{v}}+W_{T T}^{Z_{a}}\right)-\rho_{T}^{\prime}\left(W_{T}^{\prime \gamma_{a}}+a_{v} W_{T}^{\prime Z_{a}}\right) \\
& \left.\left.-\rho_{L T}^{\prime}\left(\left(W_{L T}^{\prime \gamma_{a}}+a_{v} W_{L T}^{\prime Z_{a}}\right)-\left(W_{L T}^{\prime \gamma_{v}}+a_{v} W_{L T}^{\prime Z_{v}}\right)\right)\right]\right\},
\end{aligned}
$$

where $c$ is related to the Mott cross section $\sigma_{M}$ by

$$
c=\frac{\alpha}{6 \pi^{2}} \frac{k_{2}^{l a b}}{k_{1}^{l a b} q_{\nu}^{4}}=-\frac{\eta}{2 q_{\nu}^{2}} \frac{\sigma_{M}}{3 \pi^{2} \alpha} .
$$

The structure functions $W_{\alpha}^{(\prime) B}$ contain the information on the internal hadron structure with the notation $\alpha \in\{L, T, L T, T T\}$ and $B \in\left\{\gamma_{v}, Z_{v}, \gamma_{a}, Z_{a}\right\}$, where the subscripts " $v / a$ " denote a parity conserving/violating contribution. Also parity violation in the hadronic sector has been allowed for, and the corresponding structure functions are denoted by a superscript " $\gamma_{a}$ ". It leads to the same types of structure function as via the weak axial hadron current $\left(Z_{a}\right)[\mathrm{KuA} 97]$.

In case, no analysis of the final state is performed, one sums over all final states. Then the interference terms $L T$ and $T T$ vanish, and one obtains for the inclusive cross section for an unpolarized target

$$
\begin{aligned}
\Sigma(h)= & d^{2} \sigma^{\gamma+Z} / d k_{2}^{l a b} d \Omega_{k_{2}}^{l a b} \\
= & c\left\{\rho_{L}\left(F_{L}^{\gamma_{v}}+a_{v} F_{L}^{Z_{v}}\right)+\rho_{T}\left(F_{T}^{\gamma_{v}}+a_{v} F_{T}^{Z_{v}}\right)-\rho_{T}^{\prime} a_{a} F_{T}^{\prime Z_{a}}\right. \\
& \left.+h\left[\rho_{L} a_{a} F_{L}^{Z_{v}}+\rho_{T} a_{a} F_{T}^{Z_{v}}-\rho_{T}^{\prime}\left(F_{T}^{\prime \gamma_{a}}+a_{v} F_{T}^{\prime Z_{a}}\right)\right]\right\} .
\end{aligned}
$$

One should note that the helicity dependent part of this expression is a direct measure of the total parity violating contribution which arises from both, hadronic parity violation and from electroweak interference. It can be determined from the longitudinal asymmetry

$$
\mathcal{A}=\frac{1}{2 h \Sigma_{0}}\left(\Sigma_{+}-\Sigma_{-}\right)
$$

where

$$
\Sigma_{0}=\frac{1}{2}\left(\Sigma_{+}+\Sigma_{-}\right), \quad \text { and } \quad \Sigma_{ \pm}=\Sigma( \pm h) .
$$

One may split the asymmetry into contributions from hadronic and electroweak parity violation, resulting in

$$
\mathcal{A}=\mathcal{A}^{\gamma_{a}}+\mathcal{A}^{Z}
$$

where

$$
\mathcal{A}^{\gamma_{a}}=-\frac{\rho_{T}^{\prime} W_{T}^{\prime \gamma_{a}}}{\rho_{L} W_{L}^{\gamma_{v}}+\rho_{T} W_{T}^{\gamma_{v}}},
$$




$$
\begin{aligned}
\mathcal{A}^{Z} & =\frac{\rho_{L} a_{a} W_{L}^{Z_{v}}+\rho_{T} a_{a} W_{T}^{Z_{v}}-\rho_{T}^{\prime} a_{v} W_{T}^{\prime Z_{a}}}{\rho_{L} W_{L}^{\gamma_{v}}+\rho_{T} W_{T}^{\gamma_{v}}} \\
& =\frac{G_{F}}{2 \sqrt{2}} \frac{q_{\mu}^{2}}{\pi \alpha} \frac{\rho_{L} W_{L}^{Z_{v}}+\rho_{T} W_{T}^{Z_{v}}+\rho_{T}^{\prime}\left(1-4 \sin ^{2} \theta_{W}\right) W_{T}^{\prime Z_{a}}}{\rho_{L} W_{L}^{\gamma_{v}}+\rho_{T} W_{T}^{\gamma_{v}}}
\end{aligned}
$$

The term proportional to $\rho_{T}^{\prime}$ is largely suppressed, since $\sin ^{2} \theta_{W}=0.232$.

\section{Selected Reactions on the Deuteron}

In order to illustrate the various facets of electroweak processes, I have chosen the deuteron, which one might consider a personal bias. However the two-body system has an undisputable preference, namely, its simple structure allows the most exact treatment, at least in the nonrelativistic domain. In the following, I will discuss a variety of specific reactions on the deuteron with special emphasis on the study of subnuclear degrees of freedom. I will begin with the electromagnetic deuteron break-up where these subnuclear d.o.f. appear either implicitly in the case of meson d.o.f. in the form of effective operators, the meson exchange currents (MEC), or explicitly in the case of isobar d.o.f. as nuclear isobar configurations (IC). Later on, also explicit meson d.o.f. will appear, when I will discuss specific meson production reactions.

\subsection{Electromagnetic Break-up}

The special role of the electromagnetic deuteron break-up is a consequence of (i) the already mentioned simple structure, and (ii) the specific features of the electromagnetic probe as discussed in the preceding section. The continued interest in this process has persisted over more than sixty years because the two-body system is in fact a unique laboratory [ArS 91]. With respect to the study of subnuclear d.o.f., one may summarize the evidence for MEC and IC in $\gamma^{(*)}+d \rightarrow p+n$ as follows:

(i) The strongest MEC contributions appear in $E 1$ in $d(\gamma, N) N$ where they dominate the cross section above $100 \mathrm{MeV}$, but they are mostly covered by the Siegert operator (see Sect. 3.4).

(ii) A clear signature of MEC is furthermore observed in $M 1$ in $d\left(e, e^{\prime}\right) n p$ near break-up threshold, particularly strong if not dominant at higher momentum transfers.

(iii) In the $\Delta$ region, one finds the strongest manifestation of IC.

Now I will illustrate these findings by a few examples.

\subsubsection{Backward Electrodisintegration near Threshold}

I will start with an early case of clear evidence of $\pi$ meson exchange current in electrodisintegration near threshold, namely the inclusive process $d\left(e, e^{\prime}\right) p n$, where no analysis is made of the hadronic final state [SiB 79]. The threshold region is dominated by the excitation of the antibound ${ }^{1} S_{0}$ resonance in $N N$ scattering at very low energies. It is a process inverse to thermal $n$ - $p$ radiative capture, which proceeds via $M 1$ transition and where MEC and IC give almost a 10 percent enhancement [RiB 72, Are 81b]. The advantage of electron scattering, allowing the momentum transfer to be varied independently, becomes apparent here, since the relatively small effect of subnuclear d.o.f. in the real photon process can be amplified considerably. The reason for this lies in the fact that with increasing momentum transfer the one-body contribution drops rapidly due to a destructive interference of S- and D-wave contributions and thus the distribution of the momentum transfer onto both nucleons via 

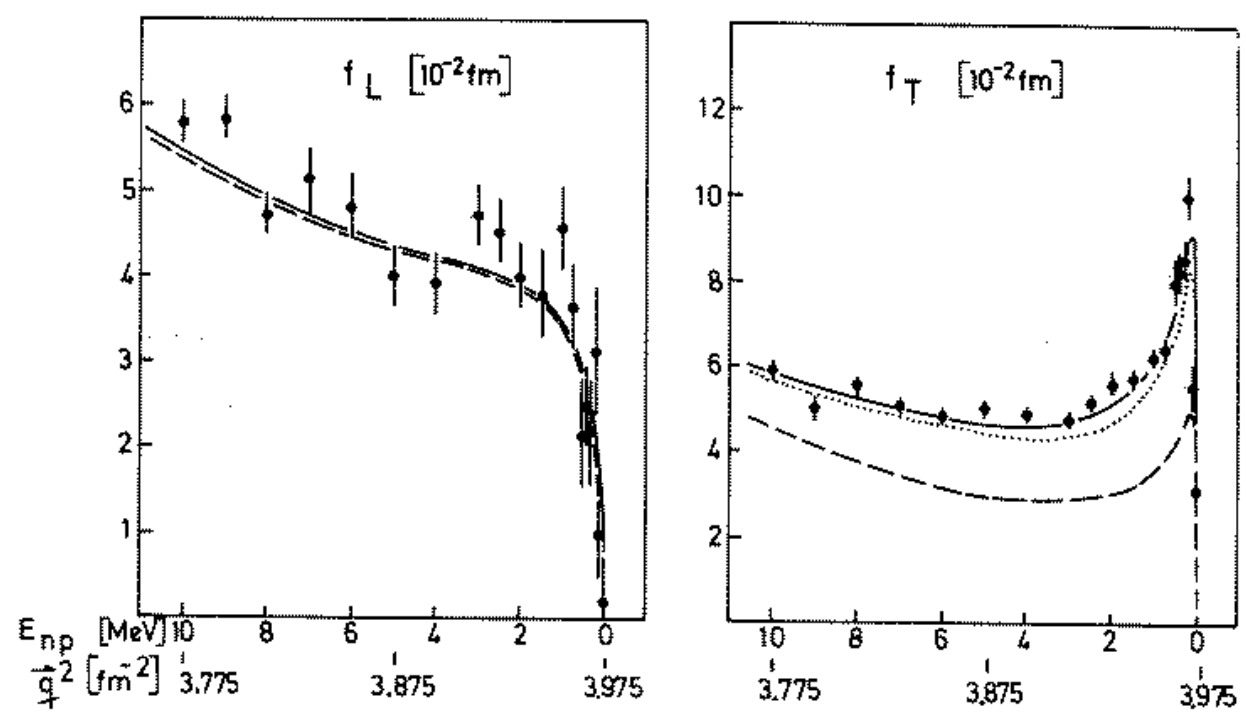

Fig. 6.1 The longitudinal (left panel) and transverse (right panel) deuteron form factors at $\vec{q}^{2} \approx 4 \mathrm{fm}^{-2}$ compared to calculations with the Hamada-Johnston potential for the normal theory (dashed) and additional $\pi$ MEC (dotted) and further added IC (full) (from [SiB 79]).

two-body operators becomes more favourable. This can be seen in Fig. 6.1, where the longitudinal and transverse form factors are shown as obtained from a Rosenbluth separation [SiB 79]. While the longitudinal form factor is well described by the classical theory, supporting the Siegert hypothesis that the charge density is little affected by exchange effects, one finds a large discrepancy in $F_{T}$ which is only resolved if MEC and IC are added.

The situation for higher momentum transfers is shown in Fig. 6.2 where the theoretical inclusive cross section is plotted at backward angles for moderate momentum transfers as recently calculated by Ritz et al. [RiG 97]. In contrast to the theoretical results shown in Fig. 6.1 , here the theoretical treatment of MEC is completely consistent, including heavy meson exchange, with the potential model, namely the Bonn OBEPQ-B model [MaH 87, Mac 89], and, in addition, all leading order relativistic contributions are taken into account. The latter can be obtained in a $(p / M)$ expansion starting from a covariant approach [AdT 89]. As briefly discussed at the end of Sect. 4, these comprise relativistic terms both in the current operators and in the wave functions including boost effects. It is obvious that for a conclusive interpretation, one has to include all corrections of the same order consistently. One readily sees in Fig. 6.2 again the strong enhancement due to MEC, but in addition one notes sizable relativistic contributions. It is clear from this result that already at low excitation energies relativistic effects may become important and have to be considered in a quantitative comparison of theory with experiment.

Such a comparison, based on the results for three OBEPQ versions of the Bonn potential with experimental data, is shown in Fig. 6.3. Here the calculation has been extended to the high momentum data of [ArB 90], although this kinematic region is beyond the limits of validity of the $(p / M)$ expansion. Between $-q_{\mu}^{2}=10$ and $30 \mathrm{fm}^{-2}$ one finds a systematic and increasing overestimation of the data by the theory, whereas above $30 \mathrm{fm}^{-2}$ the overestimation is much less pronounced and more constant. The variation of the different potential versions is comparably small, except for the very highest momentum transfers considered.

In summary, a good agreement with experimental data is achieved at low and intermediate 

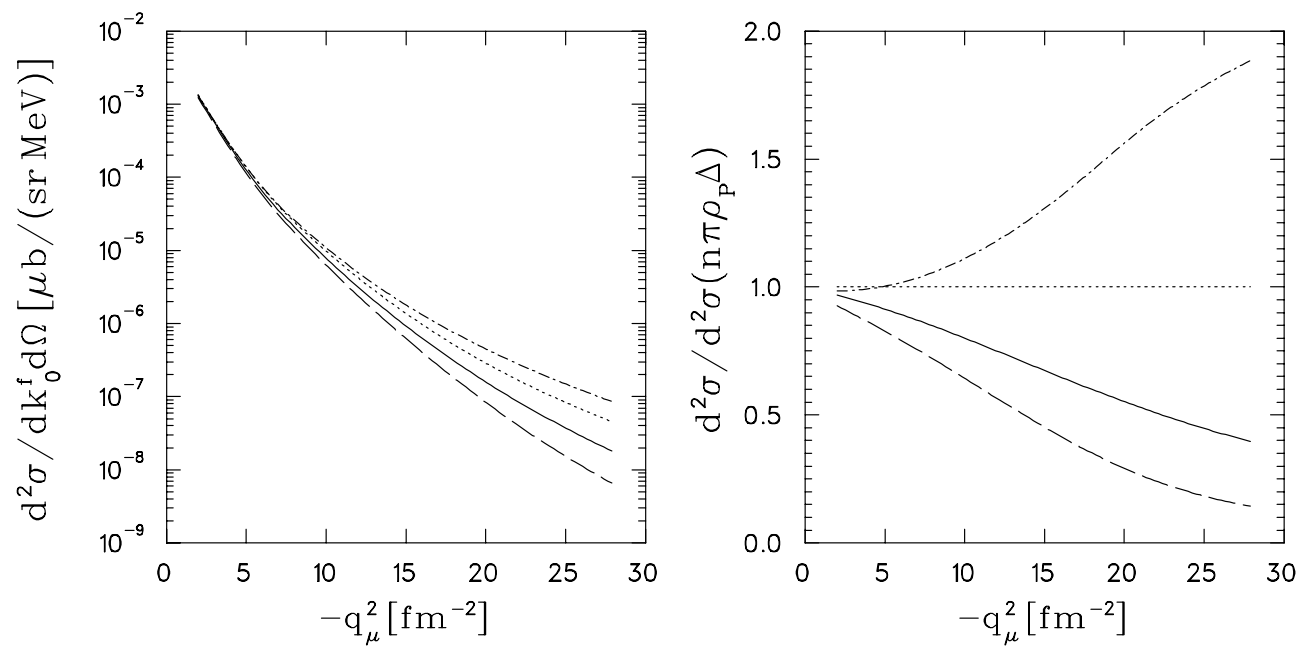

Fig. 6.2 Deuteron electrodisintegration near threshold for $E_{n p}=1.5 \mathrm{MeV}$ at backward angles $\left(\theta_{e}=155^{\circ}\right)$. Left: absolute values; Right: relative with respect to the nonrelativistic theory including the conventional $\pi$ and $\rho$ MEC and $\Delta$ contribution. Notation of the curves: dotted: nonrelativistic theory including the conventional $\pi$ and $\rho$ MEC and $\Delta$ contribution; dash-dotted: in addition relativistic one-body contributions including kinematic boost; dashed: further added all relativistic contributions to $\pi$ MEC; full: complete calculation (from [RiG 97]).

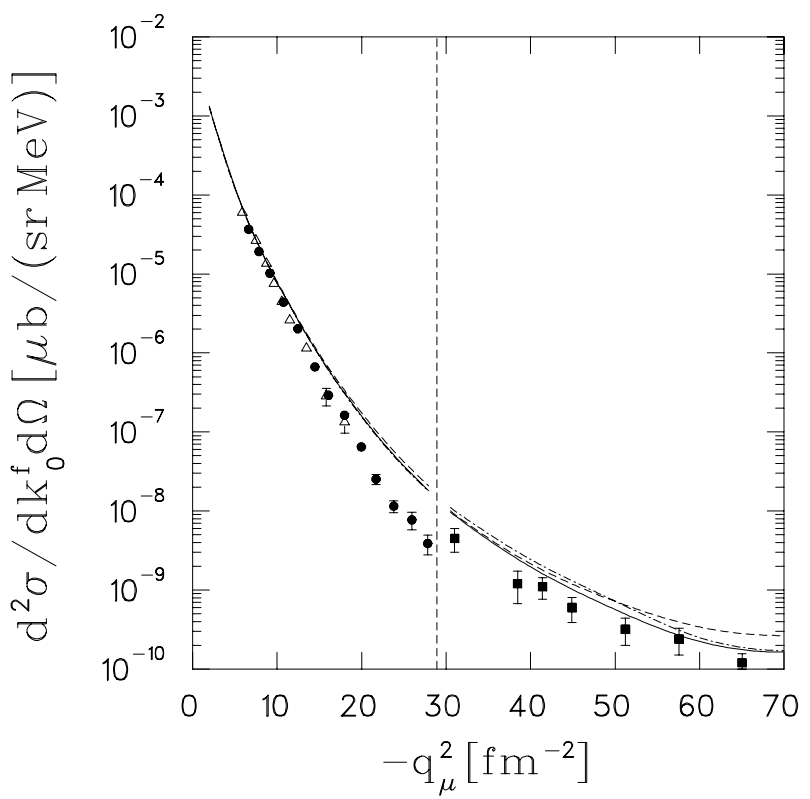

Fig. 6.3 Potential model dependence of deuteron electrodisintegration near threshold and comparison with experiment. Experimental data points: open triangles: [BeJ 81], filled circles: [AuC 85] $\left(\theta_{e}=155^{\circ}\right.$, averaged over energies $0 \leq E_{n p} \leq 3 \mathrm{MeV}$; theory for $\left.E_{n p}=1.5 \mathrm{MeV}\right)$; filled squares: [ArB 90] $\left(\theta_{e}=180^{\circ}\right.$, averaged over energies $0 \mathrm{MeV} \leq$ $E_{n p} \leq 10 \mathrm{MeV}$; theory for $\left.E_{n p}=5 \mathrm{MeV}\right)$. Notation of the curves: full: Bonn OBEPQB potential, dashed: OBEPQ-A potential, dash-dotted: OBEPQ-C potential (from [RiG 97]). 
momentum transfers. Relativistic contributions are sizable and have to be included. In particular, the noted difference in nonrelativistic calculations between taking the e.m. nucleon form factors in the Sachs or in the Dirac-Pauli parametrization disappears essentially [WiB 93, BeW 94]. But at higher momentum transfers, a completely covariant approach without resort to a $(p / M)$ expansion is necessary.

\subsubsection{Signature of a $\Delta \Delta$ Component in Electrodisintegration}

One important consequence of the internal nucleon structure is the presence of small nuclear wave function components where one or more nucleons are internally excited, i.e., exist in an isobar state like a $\Delta(1232)$ or a Roper resonance $N(1440)$ [ArW 72, Gre 76, WeA 78]. This is a consequence of the fact, that during a collision of two nucleons there is a nonvanishing probability for the internal excitation of one or both nucleons. Because of the relatively large excitation energy $(\geq 300 \mathrm{MeV}$ ) compared to typical nuclear energies, such virtual excitations have a correspondingly short lifetime. Thus they will modify the nuclear wave function mainly in the shorter range domain[WeA 78]. Since these isobar configurations (IC) have a rather small probability, their influence is difficult to detect. In any case, evidence for them will only be indirect since they are not directly observable. However, under certain favourable conditions they may lead to sizable effects in processes which are sensitive to the shorter range region, i.e., in higher momentum transfer processes. I would like to show one example for the double $\Delta$ component in the deuteron, namely the charge structure function $f_{L}$ of $d\left(e, e^{\prime} p\right) n$. In lowest order, one has no contribution to the charge density from meson exchange and the relativistic contributions are very small. Thus the largest effect from subnuclear d.o.f. arises from IC.

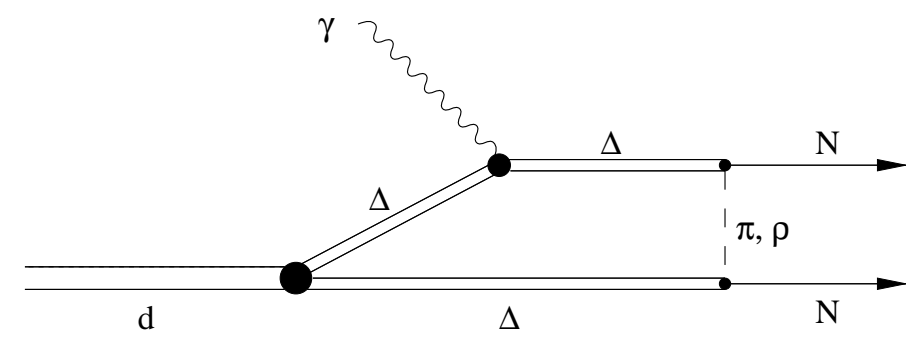

Fig. 6.4 Diagrammatic representation of the $\Delta \Delta$ contribution to the longitudinal structure function $f_{L}$.

Since the lowest order $\pi$-MEC charge density vanishes (see Sect. 4.2), effects from subnuclear d.o.f. can arise only from IC. In view of the fact, that the charge excitation of a $\Delta$, which has to proceed via $C 2$ is largely suppressed, the only contribution of IC to the longitudinal structure function $f_{L}$ comes from the diagonal $\Delta$ charge density, states as sketched in the diagram of Fig. 6.4. At low energy transfer, the contribution is expected to be relatively small. However, with increasing energy transfer its relative importance is enhanced considerably because of the increase of the double $\Delta$ component in the final state. This behaviour is shown in Fig. 6.5. Although the $\Delta \Delta$ components in the deuteron have a total admixture probability of less than 1 percent, they produce about a 15 percent enhancement of $f_{L}$ at 200 $\mathrm{MeV}$, while at $100 \mathrm{MeV}$ their effect is still negligible. 

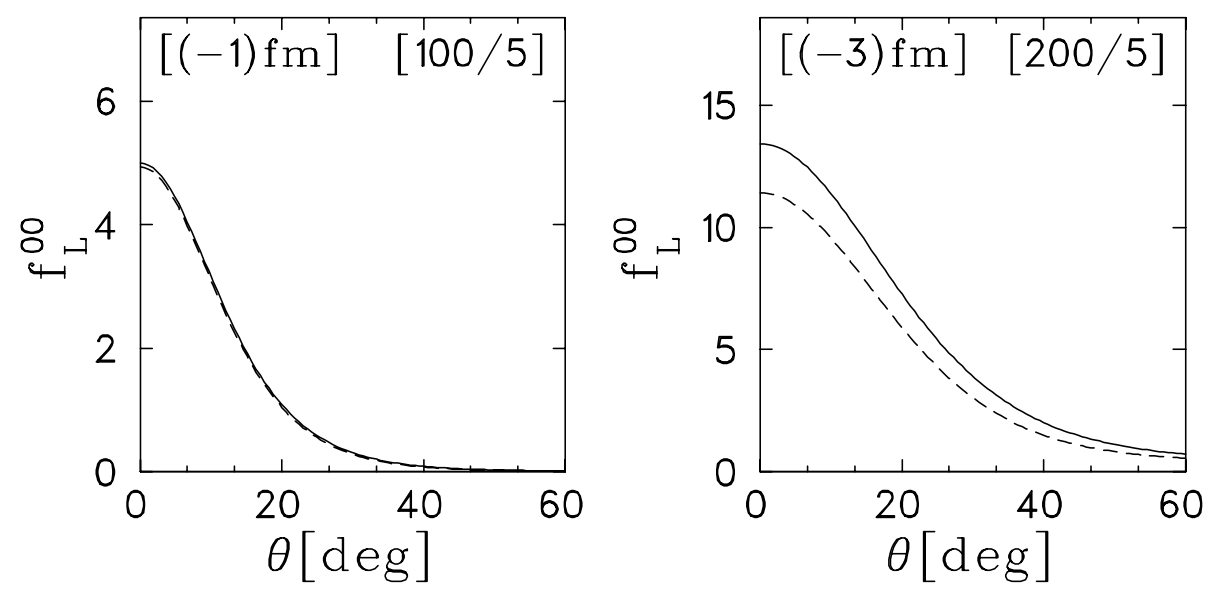

Fig. 6.5 Longitudinal structure function in the $\Delta$ region for $E_{n p}=100$ and $200 \mathrm{MeV}$ at $q^{2}=5 \mathrm{fm}^{-2}$ without (dashed) and with (full) $\Delta \Delta$ component. The top left inset "[(-n) $\mathrm{fm}]$ " indicates the unit $\left[10^{-n} \mathrm{fm}\right]$ for $f_{L}^{00}$ and the top right inset means " $\left[E_{n p} / q_{c . m}^{2}\right]$ ", where $E_{n p}$ in $[\mathrm{MeV}]$ and $q^{2}$ in $\left[\mathrm{fm}^{-2}\right]$.

\subsubsection{Heavy Meson Exchange and Consistent Relativistic Effects in Photodis- integration below Pion Threshold}

Now I will turn to a discussion of the influence of heavy meson exchange on deuteron photodisintegration with inclusion of competing relativistic effects in the one-body and pion exchange sector as presented recently by Ritz et al. [RiA 97]. The details of the calculational framework can be found in [RiG 97]. As an example, I show in Fig. 6.6 the unpolarized differential cross section at four representative photon energies $E_{\gamma}=4.5,40,100$, and 140 $\mathrm{MeV}$ covering the region between the maximum of the total cross section and pion production threshold. In order to distinguish the different influences from pion, rho, and other heavy meson exchanges, their effects are shown in separate panels for each observable and each energy. In addition, an overview is shown as well as the potential model dependence with respect to the versions $\mathrm{A}, \mathrm{B}$, and $\mathrm{C}$ of the Bonn OBEPQ potential model [MaH 87, Mac 89].

The overview shows that in the maximum of the total cross section, at $4.5 \mathrm{MeV}$, the differential cross section is dominated by the nonrelativistic one-body current, while only the nonrelativistic $\pi$ MEC gives a 10 percent enhancement, predominantly in $E 1$. Obviously, relativistic effects and heavy mesons are negligible as well as the potential model dependence.

At the next higher energy $(40 \mathrm{MeV})$, the nonrelativistic $\pi$ MEC becomes comparable to the one-body current. All other contributions give a small overall reduction, somewhat more pronounced in forward and backward direction. However, if one looks at the separate contributions, one notices a subtle destructive interference of different larger effects. First, relativistic $\pi$ MEC gives a slight reduction in the maximum but leaves the forward and backward directions almost unchanged. Next, with respect to the $\rho$, one sees a strong forward and backward reduction from the Pauli current (see [RiG 97] for the distinction between Pauli and Dirac current for the $\rho$ MEC), whereas the Dirac contribution, which is often neglected, mainly leads to a sizable enhancement in the maximum which, however, is largely cancelled by the additional heavy mesons. Finally, one finds a small potential model dependence of a few percent.

Considering now the two higher energies (100 and $140 \mathrm{MeV}$ ), one readily notices a dramatic increase of relativistic effects. First a sizable reduction appears from the relativistic one-body current showing the well known effect of diminishing the differential cross section 

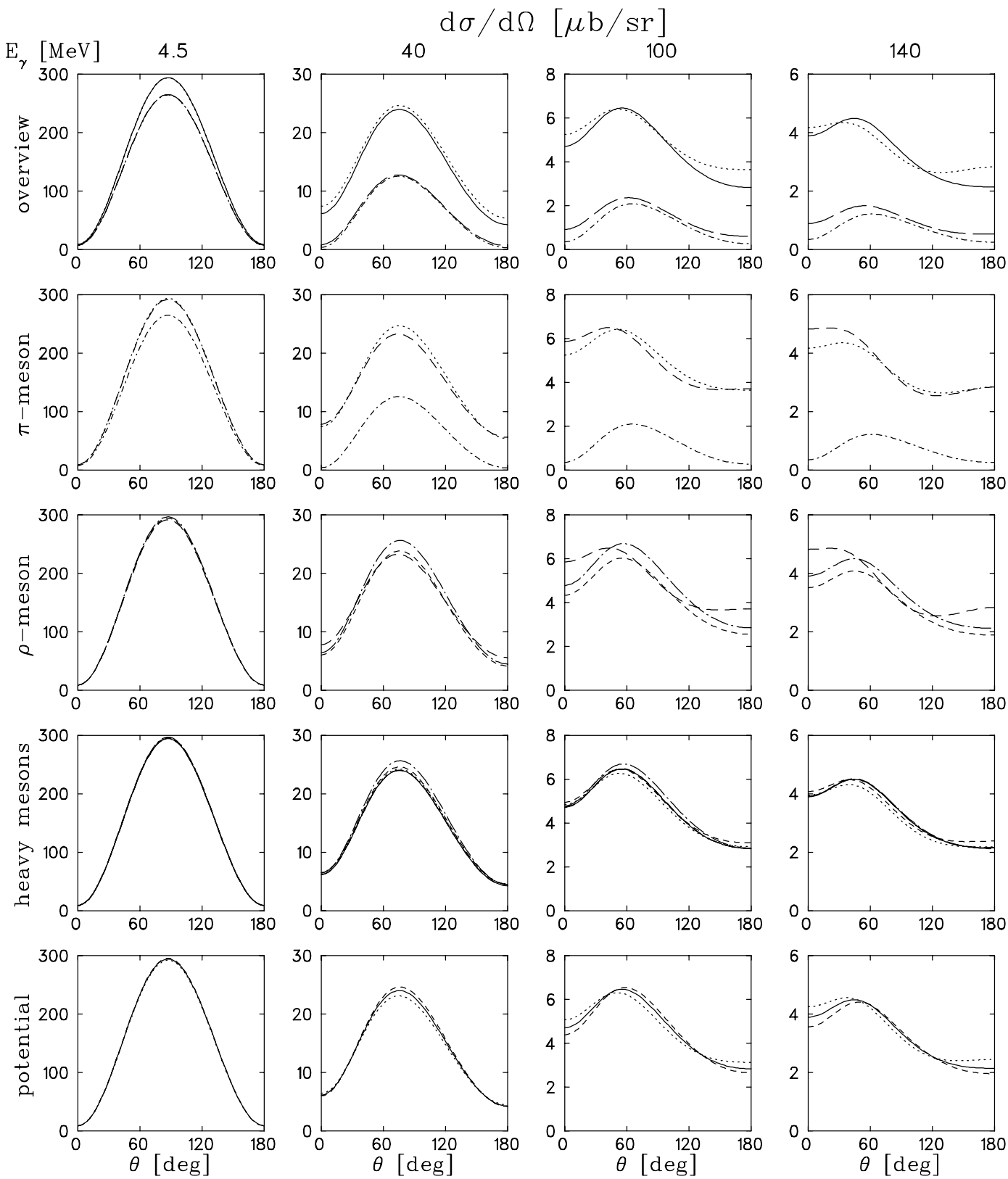

Fig. 6.6 The differential cross section of $d(\gamma, p) n$ for various laboratory photon energies. Notation of the curves: (1) overview: nonrelativistic one-body current (long-dashed); relativistic one-body current (dash-dotted); nonrelativistic $\pi$ MEC added (dotted); total result (full); (2) $\pi$ meson: relativistic one-body current (dash-dotted); nonrelativistic $\pi$ MEC added (dotted); relativistic $\pi$ MEC including retardation (dashed); (3) $\rho$ meson: relativistic one-body plus complete $\pi$ MEC (dashed); Pauli MEC (short-dashed); Dirac MEC (long-dashed-dotted); (4) heavy meson: relativistic one-body current plus complete $\pi$ and full $\rho$ MEC (long-dash-dotted); $\delta$ MEC (dotted); $\omega$ MEC (short-dashed); $\sigma$ MEC (dashed); $\eta$ MEC (full); (5) potential: Bonn OBEPQ version B (full); version A (short-dashed); version C (dotted). 

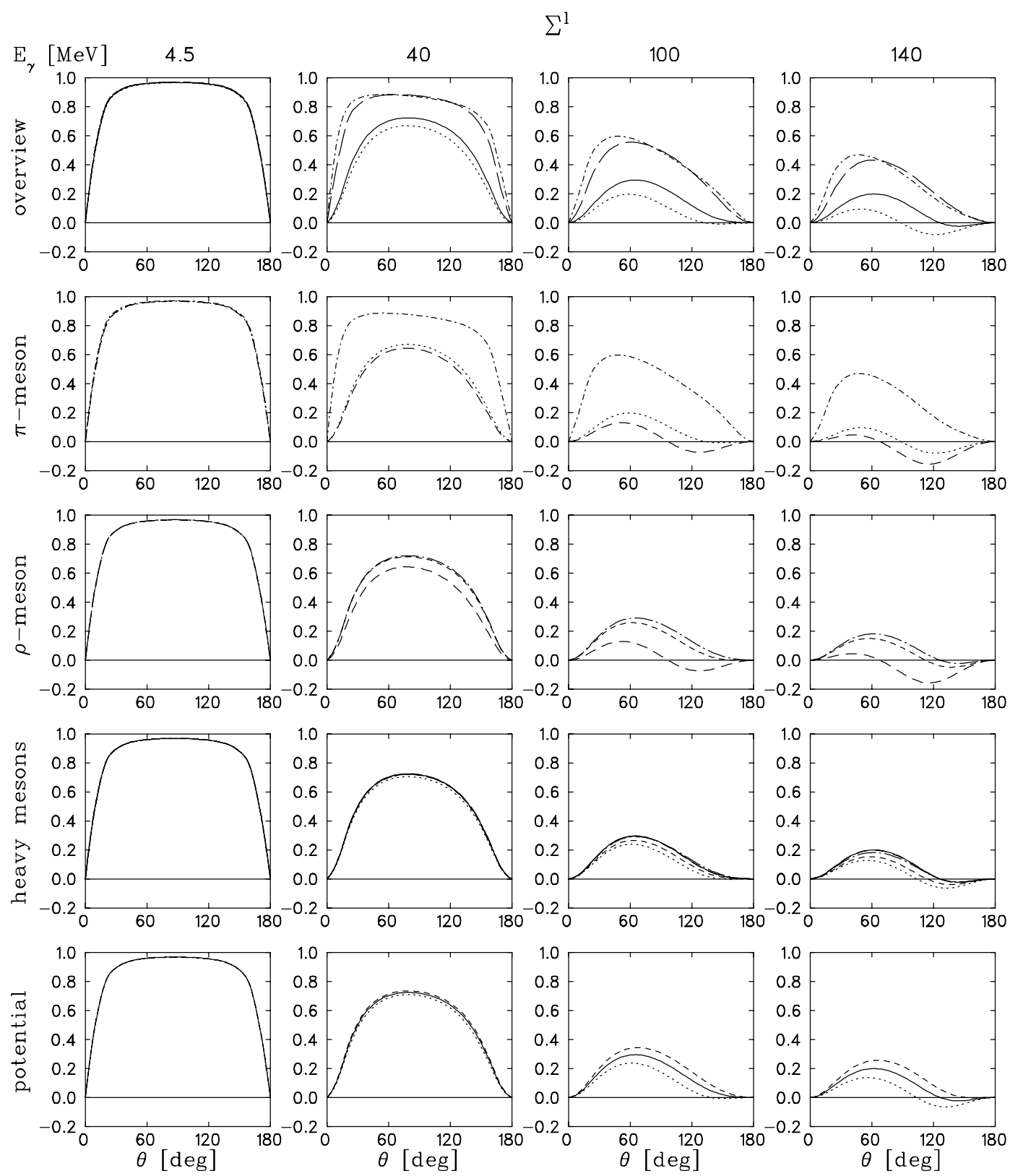

Fig. 6.7 The photon asymmetry $\Sigma^{l}$ for $d(\vec{\gamma}, p) n$. Notation of the curves as in Fig. 6.6. 
at forward and backward angles, which comes mainly from the dominant spin-orbit current [CaM 82]. The further reduction from the remaining contributions is again the result of a strong destructive interference of larger contributions. In fact, first the relativistic $\pi$ MEC surprisingly enhances the cross section in forward direction, while then the $\rho$ Pauli current results in a drastic reduction at both extreme angles. The Dirac contribution gives again an overall enhancement but of smaller size. This effect of the Dirac $\rho$ current is somehow surprising, because it is roughly of the same size as that of the Pauli current, whereas from the size of the coupling constants one would have expected a suppression by a factor of about 50. The additional heavy mesons beyond the $\rho$ meson show a much smaller influence. Their individual contributions are remarkably big (up to $\sim 5 \%$ ), in particular compared to their role in the parametrization of the $N N$ force and their importance in the electrodisintegration of the deuteron [RiG 97]. Most prominent is the effect of the $\delta$ meson leading to a reduction of the differential cross section. However, looking at the net result, the heavy meson exchanges tend to cancel each other. With respect to the potential model dependence, one sees now a larger variation, in particular also at forward and backward angles which increases with energy.

The photon asymmetry for linearly polarized photons $\Sigma^{l}$, shown in Fig. 6.7, is very sensitive to two-body effects, as is long known [ArS 91]. At $4.5 \mathrm{MeV}$ only the nonrelativistic one-body current contributes to $\Sigma^{l}$ and no potential model dependence appears. Then at 40 $\mathrm{MeV}$, the nonrelativistic $\pi$ MEC becomes sizable as well as the Pauli $\rho$ current. All other effects, relativistic one-body and $\pi$ MEC, Dirac $\rho$ and additional heavy meson effects are very small, as is the potential model dependence. At higher energies the relativistic onebody current as well as the relativistic $\pi$ MEC become important, too. The first leads to a sizable reduction of the photon asymmetry, the latter to a smaller increase. The $\rho$ MEC increases the photon asymmetry, of which the Pauli current is the most dominant part while the Dirac current is comparably small, although its size increases with the photon energy. The influence of the various heavy meson exchanges are much more pronounced than in the differential cross section, mainly coming from the $\delta$ MEC. But again the various heavy mesons tend to interfere destructively. The potential dependence is quite large at 100 and $140 \mathrm{MeV}$, where the OBEPQ version A yields the biggest asymmetry, version B intermediate values, and version $\mathrm{C}$ the lowest photon asymmetry. Thus one might be tempted to single out one potential against the others by comparison with experimental data. However, one has to be careful in such a reasoning [BlB 91], because, before drawing definite conclusions as to which model should be preferred, one has to study in detail the remaining theoretical uncertainties due to the strength of the dissociation and isobar currents. Here, the additional independent measurement of the unpolarized cross section and the vector target asymmetry $T_{11}$ could help in fixing the respective strengths of these contributions.

\subsubsection{Pion Retardation in Photodisintegration above $\pi$ Threshold}

Photodisintegration in the $\Delta$ resonance region is of particular interest for the study of the $N \Delta$ interaction. However, all models developed so far are unable to describe in a satisfactory manner the experimental data over the whole $\Delta$ resonance region (for a review see [ArS 91]). Among the most sophisticated approaches are the unitary three-body model of Tanabe and Ohta [TaO 85] and the coupled channel approach (CC) of Wilhelm and myself [WiA 93]. In both models, all free parameters were fixed in advance by fitting $N N$ and $\pi N$ scattering, and $\pi$ photoproduction on the nucleon. Consequently, no adjustable parameters remained for deuteron photodisintegration. However, it turned out that both approaches considerably 
underestimated the total cross section in the $\Delta$ region by about 20-30\% [TaO 85, WiA 93]. Another failure was the wrong prediction of the shape of differential cross section and photon asymmetry, especially at photon energies above $300 \mathrm{MeV}$ [ $\mathrm{TaO}$ 85, WiA 93, BlB 95].

In these calculations, one of the principal problems is the question of how to fix the magnetic $\gamma N \Delta$ strength $G_{\Delta N}^{M 1}$. In [TaO 85] and [WiA 93] it has been fitted to the $M_{1+}(3 / 2)$ multipole of pion photoproduction on the nucleon including resonance and Born contributions. When embedded into the two-nucleon system, these Born terms become part of the retarded two-body recoil and $\pi$ meson currents, respectively (Fig. 6.8). However, in the usual

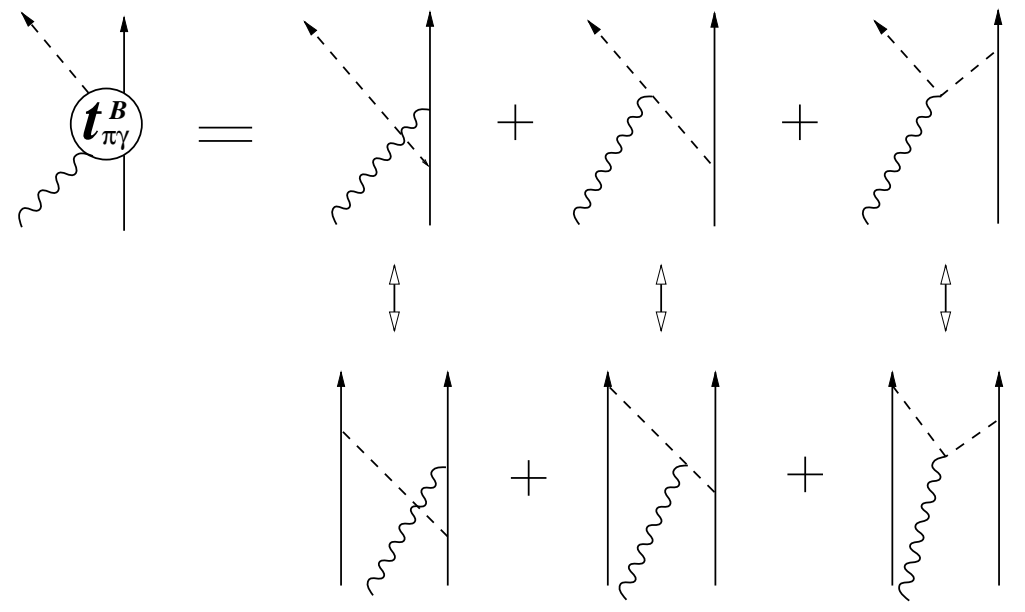

Fig. 6.8 Correspondence of Born terms of the $M_{1+}^{3 / 2}$ multipole of pion photoproduction and meson exchange current diagrams.

static calculations of MEC, the retardation of the pion is completely neglected. Furthermore, the recoil current is not present due to its cancellation against the wave function renormalization [GaH 76]. Thus, there is an inconsistency in the treatment of pion d.o.f. Indeed, it had already been conjectured in [WiA 93] that this inconsistency of using static MEC may be the origin of the observed underestimation of the total cross section in the coupled channel approach, because by incorporating the Born terms effectively into an increased $M 1-\Delta$ excitation strength, a satisfactory agreement with the data could be achieved.

In order to avoid these shortcomings, recently M. Schwamb et al. [ScA 97] have included for the first time in a coupled channel approach complete retardation in the $\pi$ exchange contributions to potential and MEC. For the retarded $N N$ potential an improved version of the energy dependent Bonn OBEPT has been chosen, which had been developed by Elster et al. [EIF 88]. It has to be renormalized via subtraction of a $N \Delta$ box graph [GrS 79, PoS 87]. The transitions between $N N$ and $N \Delta$ space are mediated by retarded $\pi$ and furthermore $\rho$ exchange, whose form factors are fixed by fitting the ${ }^{1} D_{2} N N$ partial wave. In order to ensure unitarity up to the $2 \pi$ threshold, the formation of an intermediate $N N$ state with the quantum numbers of the deuteron and a pion as spectator (denoted for simplicity by $\pi d$ channel) has been considered in addition.

The results for the total cross section are shown in Fig. 6.9. Similar to [WiA 93], the static calculation considerably underestimates the data. Inclusion of retardation in the hadronic interaction even lowers further the cross section, which is more than compensated by retardation in the $\pi$ MEC giving a strong enhancement which can be traced back essentially to the inclusion of recoil current contributions. The inclusion of the $\pi d$ channel and the $\rho \pi \gamma / \omega \pi \gamma$ MECs enhances the cross section further, so that our full calculation now gives quite a good agreement with experimental data over the whole energy range. In Figs. 6.10 and 6.11, dif- 


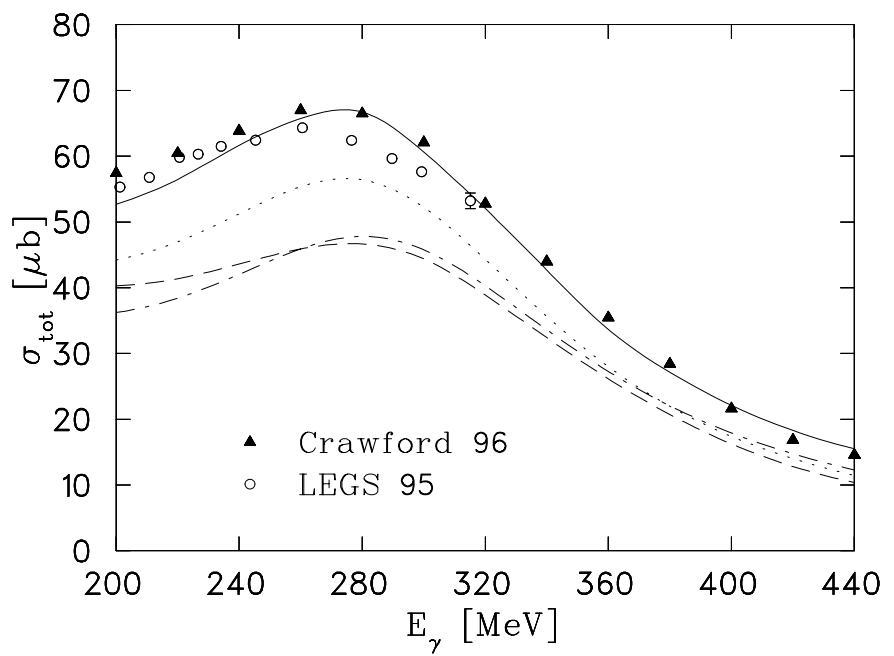

Fig. 6.9 Total cross section of $d(\gamma, p) n$. Notation: Dashed: static calculation of [WiA 93]; Dotted: static OBEPR calculation; Dash-dot: retardation switched on in the hadronic part only, but static MECs; Full: complete calculation including $\pi d$ channel and $\rho \pi \gamma / \omega \pi \gamma$ MECs (from [ScA 97]).
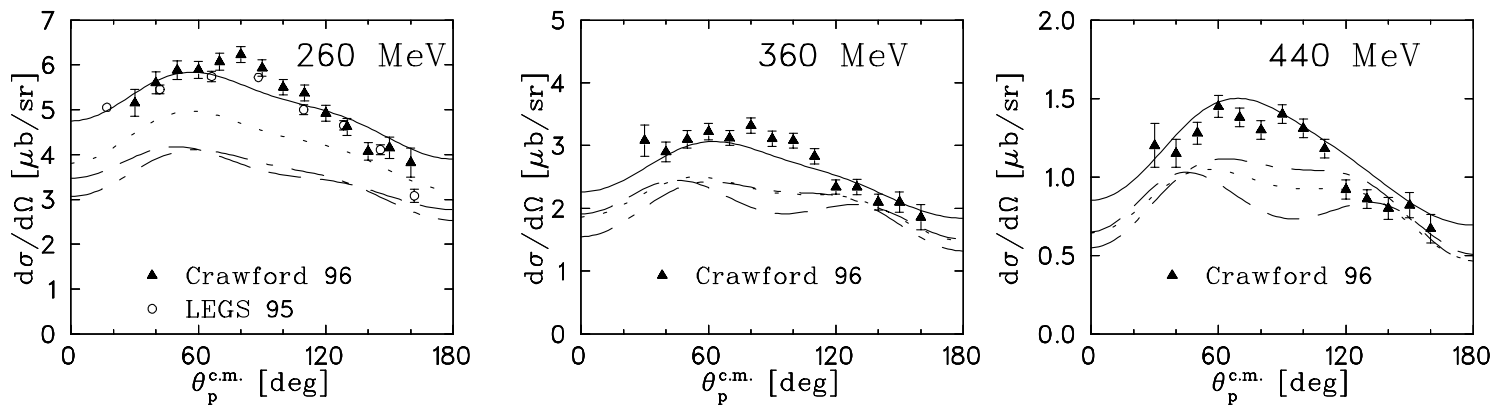

Fig. 6.10 Differential cross section of $d(\gamma, p) n$. Notation as in Fig. 6.9 (from [ScA 97]).

ferential cross section and photon asymmetry for various energies are shown. Whereas the differential cross section is in satisfactory agreement with the data, the absolute size of the asymmetry is slightly underestimated. However, in contrast to [TaO 85, WiA 93], the shape of these two observables at higher energies is reproduced quite well. In summary, complete inclusion of pion retardation is necessary, showing a strong influence on cross section and polarization observables and yielding a much improved description of experimental data.

\subsection{Photoproduction of Mesons}

Meson photoproduction is the primary absorptive process on the nucleon. It proceeds mainly through the intermediate excitation of a nucleon resonance and gives important information on the internal nucleon structure. Therefore, it provides stringent tests for any kind of hadron models. But one has to keep in mind, that the elementary process contains besides the resonance contributions also background or Born terms and that both contributions are coupled dynamically. In view of the fact, that the background is largely phenomenological, the extraction of the interesting resonance properties is not unique [WiW 96, WiW 98]. The reason for this is, that one can arbitrarily shift resonance contributions to the background and vice versa via unitary transformations without changing the observable quantities.

Meson photo- and electroproduction on light nuclei is primarily motivated by the following 

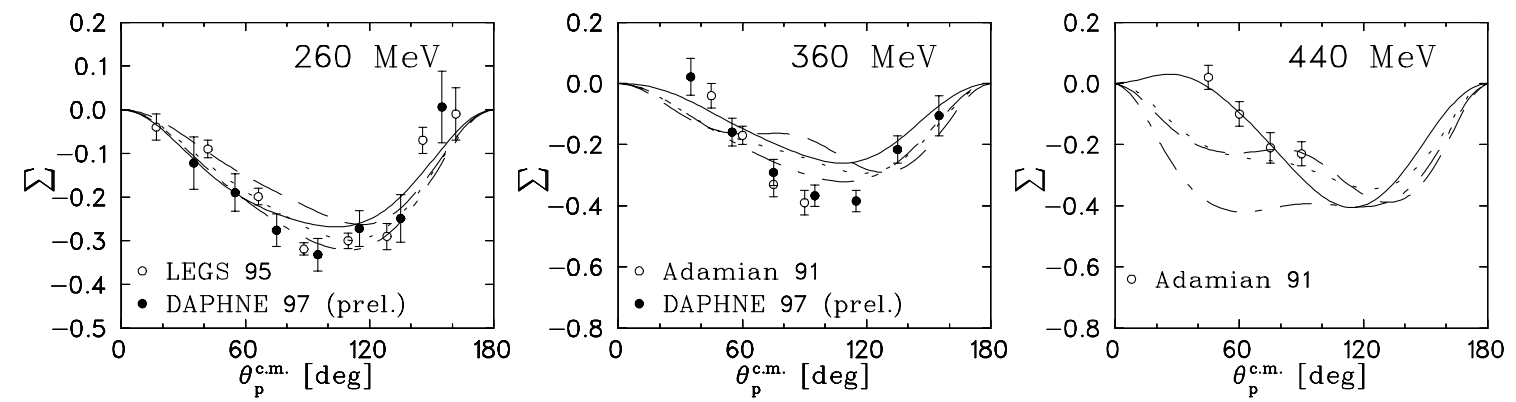

Fig. 6.11 Photon asymmetry $\Sigma^{l}$ of $d(\vec{\gamma}, p) n$. Notation of the curves as in Fig. 6.9 (from [ScA 97]).

possibilities: (i) study of the elementary neutron amplitude in the absence of a neutron target, (ii) investigation of medium effects, i.e., possible changes of the production operator in the presence of other nucleons, and (iii) it provides an interesting means to study nuclear structure. As an illustration of these various aspects, I will present recent results on coherent and incoherent pion and eta photoproduction on the deuteron.

\subsubsection{Coherent Pion Photoproduction in the $\Delta(1232)$ Region}

Recently, Wilhelm and myself have studied coherent $\pi^{0}$ production on the deuteron in the $\Delta$ region. In the first work in [WiA 95], we had neglected rescattering effects in order to study systematically the details of the elementary production amplitude on the one hand, and to investigate the influence of genuine two-nucleon mechanisms on the other hand. However, the comparison of theoretical predictions and experimental data gave clear indication that rescattering effects may be important. These have then been investigated in [WiA 96] using a theoretical concept which with respect to the hadronic part is based mainly on the developments of Sauer and collaborators [Sau 86, PoS 87] and is similar to the treatment of Lee et al. [BeL 81, Lee 83, LeM 85]. The model includes explicit pion, nucleon and delta degrees of freedom in a system of coupled equations for the $N \Delta, N N \pi$ and $N N$ channels. For other approaches see the references cited in [WiA 95] and the recent work of Kamalov et al. [KaT 97].

I show in Fig. 6.12 all diagrams of the process considered in the calculation of [WiA 96]. They include the impulse approximation (IA), namely the direct $\Delta$ excitation (denoted $\Delta[1]$ in Fig. 6.12), the direct and crossed nucleon pole terms ( $\mathrm{NP}[1]$ and $\mathrm{NC}[1]$, respectively), and the disconnected direct and crossed two-nucleon processes ( $\mathrm{NP}[2]$ and $\mathrm{NC}[2]$, respectively). These are the first five diagrams on the rhs of Fig. 6.12. The remaining diagrams of Fig. 6.12 describe two-body currents and rescattering effects considered in [WiA 96].

The common feature of the IA diagrams in Fig. 6.12 is that the hadronic intermediate state, either $\Delta N, N N$ or $\pi N N$, propagates freely. In principle each diagram will be accompanied by a corresponding one, where the intermediate state is subject to the hadronic interaction. Furthermore, the interaction will allow couplings between different intermediate states. However, in [WiA 96] the interaction has been restricted to the most important intermediate $N \Delta$ state resulting in the rescattering amplitude $\mathrm{R} \Delta \Delta$ and to the $N N-N \Delta$ coupling $\mathrm{R} \Delta \mathrm{N}$ in Fig. 6.12.

As first result, I show in Fig. 6.13 the total cross section. The dotted curve corresponds to the impulse approximation. Adding MECs $(\Delta[2]$ and $\mathrm{N}[2])$ gives a slight increase of a few percent in the maximum (dash-dotted curve). But by far much more important are 

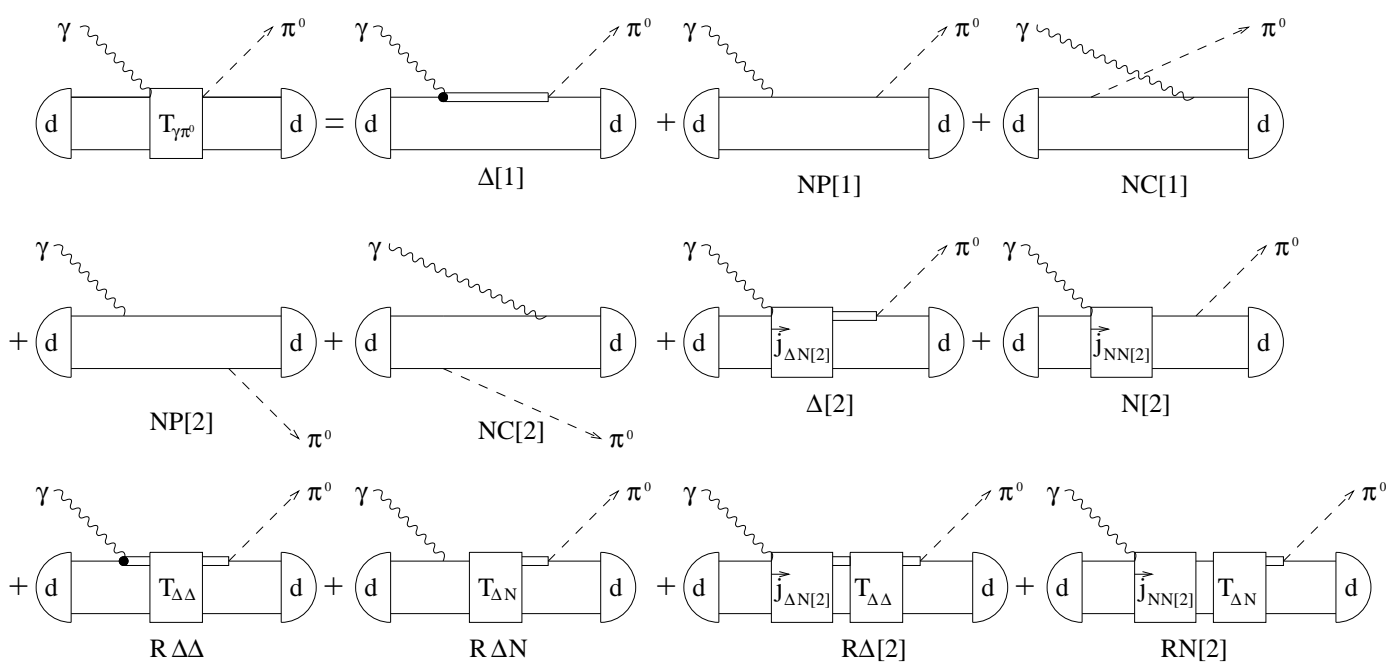

Fig. 6.12 Diagrammatic representation of the impulse approximation, two-body and rescattering contributions to $d\left(\gamma, \pi^{0}\right) d$ considered in [WiA 96].

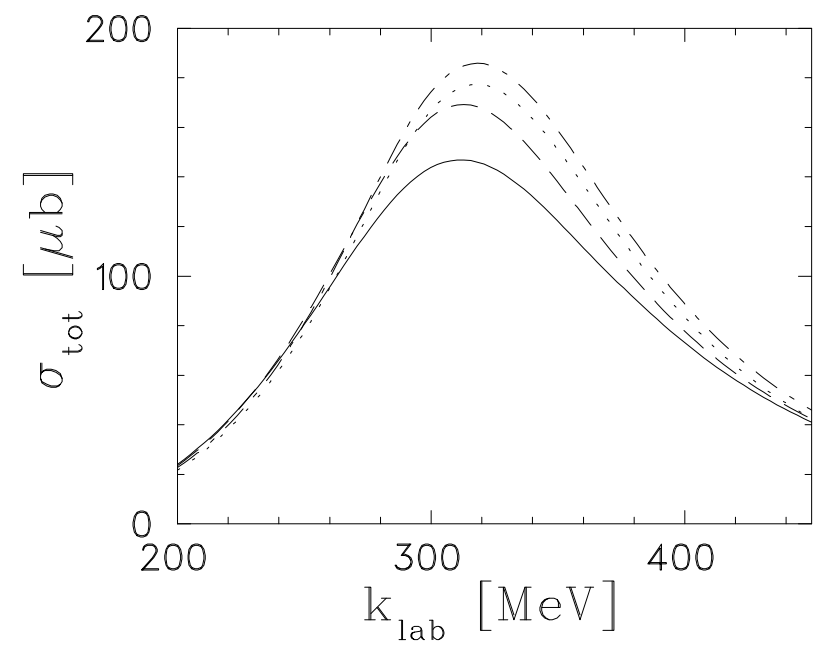

Fig. 6.13 Total cross section of $d\left(\gamma, \pi^{0}\right) d$. Notation of the curves: Dotted: impulse approximation; Dash-dot: IA plus MEC; Dashed: rescattering in Born approximation; Full: complete calculation (from [WiA 96]).

the other rescattering mechanisms. They reduce the cross section significantly and shift the maximum to a slightly lower position. Furthermore, a comparison of the full to the dashed curve clearly demonstrates that the perturbative treatment (Born approximation) is certainly insufficient. It underestimates the full dynamical effect by more than half and thus can provide a qualitative description at best.

The differential cross sections for fixed pion angles, plotted in Fig. 6.14, show the same features. For energies in the $\Delta$ region, MECs lead to a slight increase but additional pion rescattering reduces the cross section at all angles. Its influence strongly grows with the pion angle. This qualitatively agrees with what one finds in pion deuteron elastic scattering. Intuitively, one would always expect that rescattering mechanisms become more important at higher momentum transfers, i.e., for larger scattering angles at fixed energy, since rescattering provides a means to share the momentum transfer between the two nucleons.

These results clearly show that pion rescattering is significant and reduces the cross section 

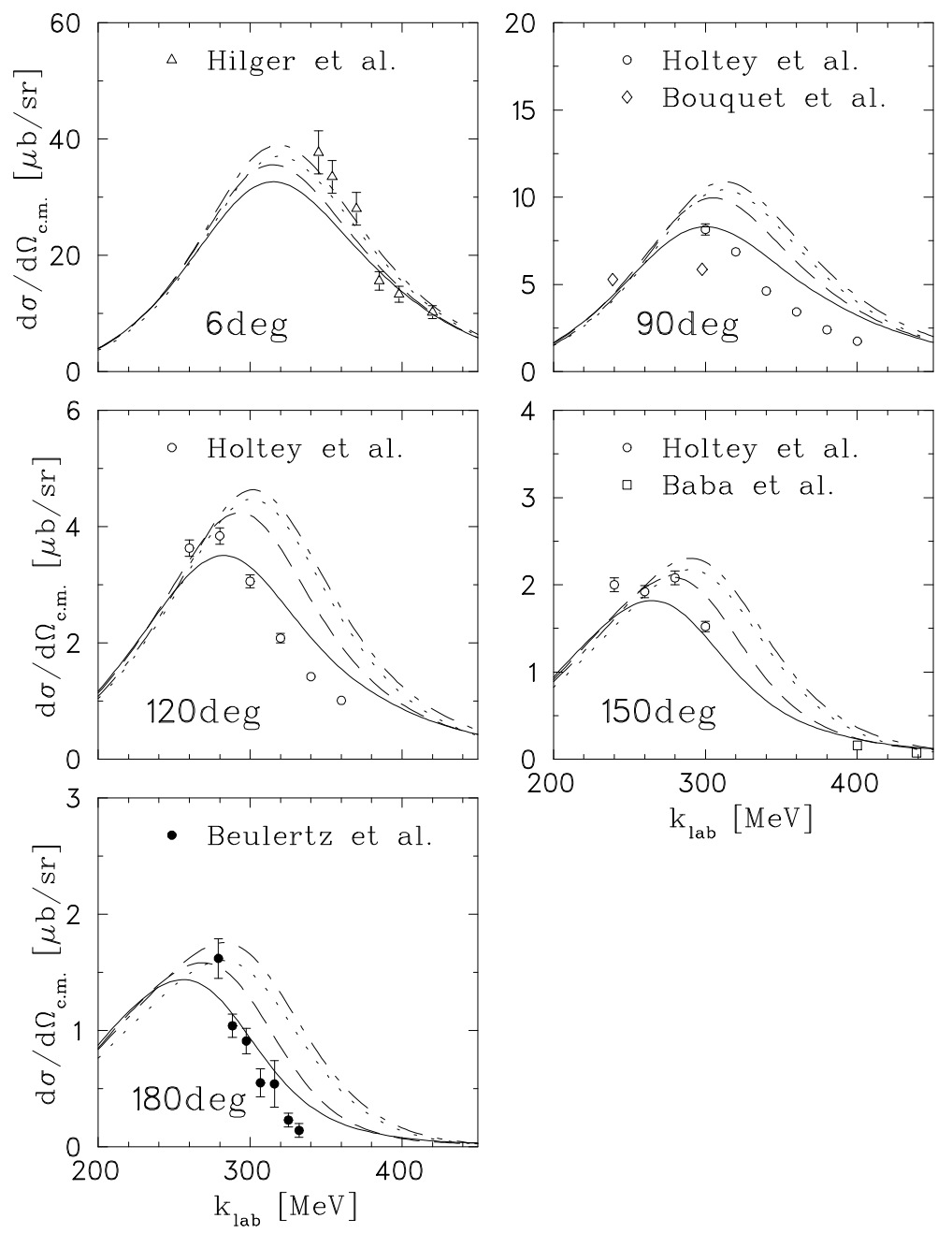

Fig. 6.14 Differential cross section of $d\left(\gamma, \pi^{0}\right) d$. Notation of the curves: Dotted: impulse approximation; Dash-dot: IA plus MEC; Dashed: rescattering in Born approximation; Full: complete calculation (from [WiA 96]). 
in the resonance region. This means, in particular with respect to a test of theoretical models for pion production amplitudes on the neutron, that one needs a reliable description for the rescattering process. Compared to experimental data, one readily finds that the sizable discrepancies without rescattering are largely reduced and that a reasonable agreement with the data is achieved. In addition polarization observables have been studied in [WiA 96]. Most of them are less sensitive to rescattering than the cross section. An exception is the tensor target asymmetry $T_{20}$ which may serve as a special tool to disentangle different reaction mechanisms (for details see [WiA 96]).

\subsubsection{Coherent Eta Photoproduction in the $S_{11}(1535)$ Region}

The special interest in the electromagnetic production of $\eta$ mesons on the nucleon is based on the fact that, being an isoscalar meson, it constitutes a selective filter for isospin $I=\frac{1}{2}$ nucleon resonances $N^{*}$. Furthermore, the e.m. $\eta$ production is dominated by the intermediate excitation of the $S_{11}(1535)$ resonance. Thus this reaction is an ideal tool for investigating the characteristic features of this resonance, which usually is difficult to separate from the other resonances because of their large widths. For example, one can study its electromagnetic transition multipoles and its decay channels, providing thus a good test for hadron models.

In a recent exploratory study, Breitmoser and myself have investigated $d\left(\gamma, \pi^{0}\right) d$ from threshold through the $S_{11}$ resonance [BrA 97]. As a first step, we had restricted ourselves to the plane wave impulse approximation (PWIA) in order to study details of the elementary reaction amplitude with respect to the yet unknown neutron properties and to study different ways of implementing the elementary amplitude in a bound system. With respect to the latter, one has to face several problems which are analogous to the corresponding pion production process on the deuteron, just discussed before. First of all, the energy of the struck nucleon on which the reaction takes place is not well defined in a bound nonrelativistic system. This leads to a severe uncertainty of the invariant mass of the $\gamma N$ subsystem of the elementary reaction, on which the elementary $t$ matrix depends. For example, the invariant mass determines the decay width of the resonance, to which the resulting cross section is very sensitive. Secondly, the elementary reaction amplitude, which usually is given in the $\gamma N$ c.m. frame, has to be transformed to an arbitrary reference frame. This may be done either by a Lorentz boost of all four momenta on which the elementary amplitude depends or by calculating the $t$ matrix with respect to an arbitrary frame right from the beginning. The last method is more general because one does not loose any terms which vanish in the $\gamma N$ c.m. frame. But in both cases, one faces again the problem of assigning an energy to the bound nucleon.

In the following, I will exclusively discuss the problem of assigning an invariant energy to the subsystem. For a bound system of two nucleons, the general expression for $W_{\gamma N}$ is, assuming the reaction to take place at nucleon " 1 ",

$$
W_{\gamma N}=\sqrt{\left(p_{10}+k_{0}\right)^{2}-\left(\vec{p}_{1}+\vec{k}\right)^{2}}=\sqrt{\left(p_{10}^{\prime}+\omega_{q}\right)^{2}-\left(\vec{p}_{1}^{\prime}+\vec{q}\right)^{2}}
$$

where $p_{1 \mu}^{(\prime)}=\left(p_{10}^{(\prime)}, \vec{p}_{1}^{(\prime)}\right)$ denotes its initial (final) four momentum. The photon momentum is denoted by $k$. In general, one has $p_{10}^{(\prime)} \neq \sqrt{M^{2}+\vec{p}^{(\prime) 2}}$ because the bound nucleon is offmass-shell. In fact, as already mentioned, the energy of an individual nucleon, bound in a nonrelativistic many-particle system, is not defined at all, i.e., there is no operator which allows to determine the energy of a bound nucleon with momentum $\vec{p}$. Only the total sum of the energies of all nucleons is a well defined quantity, e.g., for the deuteron $E_{d}^{(\prime)}=p_{10}^{(\prime)}+p_{20}^{(\prime)}$. 


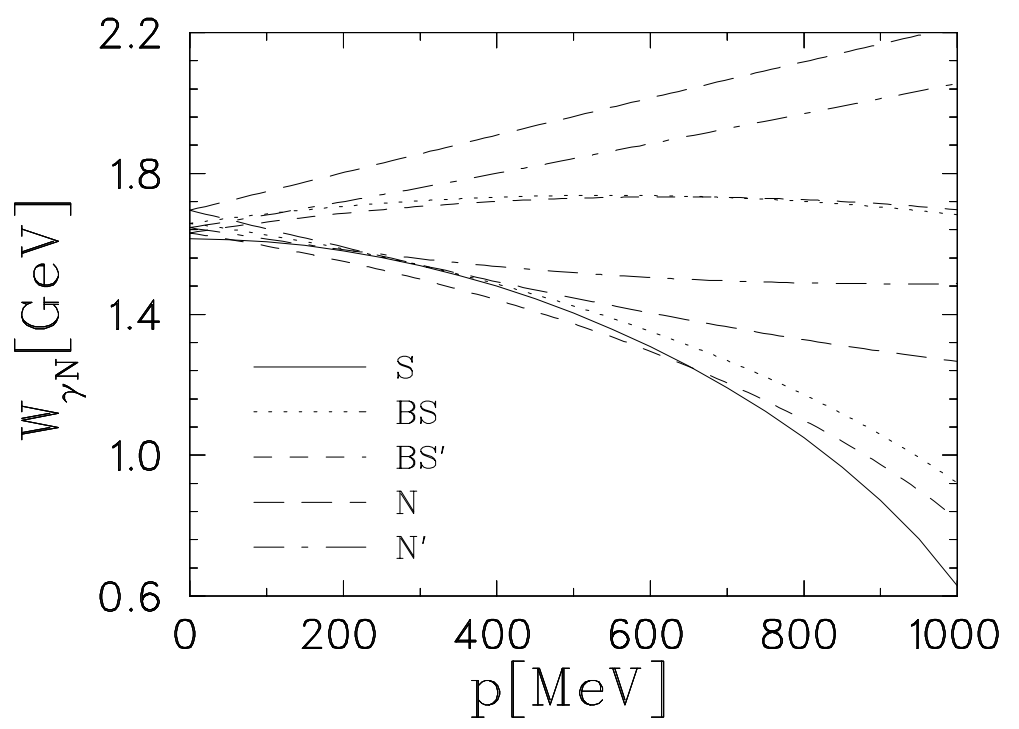

Fig. 6.15 Invariant mass $W_{\gamma N}$ of the active photon-nucleon subsystem in $d(\gamma, \eta) d$ as a function of the spectator momentum $p$. Photon energy $E_{\gamma}^{L a b}=800 \mathrm{MeV}$. Full: spectator on-shell $W_{\gamma N}^{S}$. Dotted: both initial nucleons half off-shell $W_{\gamma N}^{B S}$. Short dashed: both final nucleons half off-shell $W_{\gamma N}^{B S \prime}$. Long dashed: active initial nucleon on-shell $W_{\gamma N}^{N}$. Dash-dotted: active final nucleon on-shell $W_{\gamma N}^{N \prime}$. For the invariant mass assignments $W_{\gamma N}^{B S}, W_{\gamma N}^{B S^{\prime}}, W_{\gamma N}^{N}$, and $W_{\gamma N}^{N^{\prime}}$, two curves show the borderlines of the invariant mass region (from [BrA 97]).

One of many possible choices is to distribute the total energy of the deuteron equally on each of the two nucleons (Blankenbecler-Sugar choice) in the deuteron rest system, i.e., there each nucleon has the energy $M_{d} / 2$, independent of the momentum. This assignment for the initial or final deuteron state will be denoted by $W_{\gamma N}^{B S}$ and $W_{\gamma N}^{B S \prime}$, respectively, in the following discussion. Another possibility is to take the active nucleon on-shell, either before or after the interaction. The corresponding invariant masses will be denoted by $W_{\gamma N}^{N}$ and $W_{\gamma N}^{N \prime}$, respectively. As last choice, denoted by $W_{\gamma N}^{S}$, one may put the spectator nucleon onshell, i.e., $p_{20}=E_{p}$. This choice has been used, for example, in coherent $\pi^{0}$ photoproduction in [WiA 95, WiA 96]. It may be justified by the fact that the deuteron is only loosely bound, and hence the spectator acts nearly like a free nucleon.

Fig. 6.15 shows the invariant mass $W_{\gamma N}$ for these different choices as function of the spectator momentum $\vec{p}$ at fixed photon lab energy $E_{\gamma}^{L a b}=800 \mathrm{MeV}$. For the first four choices $\left(W_{\gamma N}^{B S}, W_{\gamma N}^{B S^{\prime}}, W_{\gamma N}^{N}\right.$ and $\left.W_{\gamma N}^{N^{\prime}}\right)$, the boundaries of the range spanned by the angle dependence are represented by two curves corresponding to $\vec{p}$ and $\vec{k}$ or $\vec{q}$ parallel (upper curve) and antiparallel (lower curve). One readily notes that $W_{\gamma N}^{N}$ spans the largest range, while the smaller ranges of $W_{\gamma N}^{B S}, W_{\gamma N}^{B S^{\prime}}$ and $W_{\gamma N}^{N^{\prime}}$ are compatible with each other. However, the average invariant masses nearly coincide for $W_{\gamma N}^{N}$ and $W_{\gamma N}^{N^{\prime}}$, and they show a slight increase with increasing spectator momentum, whereas they decrease for both $W_{\gamma N}^{B S}$ and $W_{\gamma N}^{B S^{\prime}}$ which, moreover, show a very similar behaviour. Finally, $W_{\gamma N}^{S}$ gives the lowest invariant mass with the strongest decrease with increasing $p$. One has to keep in mind that the main contribution to the total cross section originates from momenta $p$ below $400 \mathrm{MeV}$. But even in this region one notes a sizable difference between the various choices for the invariant mass of the active $\gamma N$ subsystem.

The influence of different choices for $W_{\gamma N}$ for the $\gamma N$ subsystem on the total cross section 


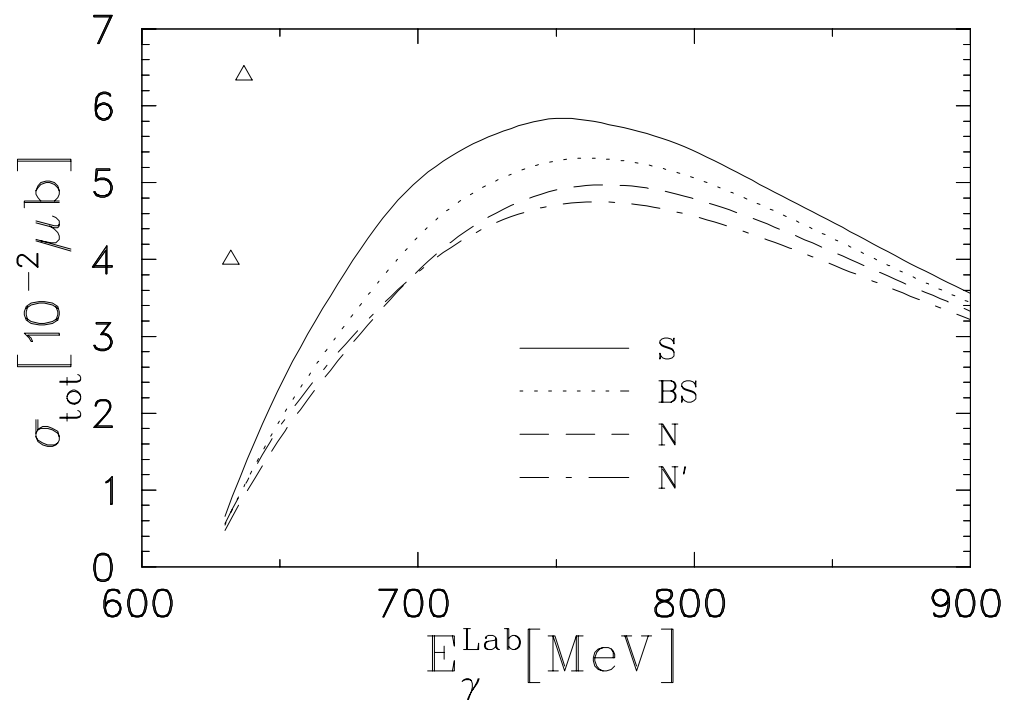

Fig. 6.16 Total cross section for $d(\gamma, \eta) d$ including only the resonance $S_{11}$ for different choices of the invariant mass of the active subsystem. Full: spectator on-shell $W_{\gamma N}^{S}$. Dotted: both initial nucleons half off-shell $W_{\gamma N}^{B S}$. Long dashed: active initial nucleon on-shell $W_{\gamma N}^{N}$. Dash-dotted: active final nucleon on-shell $W_{\gamma N}^{N^{\prime}}$. Triangles represent the upper limit of [Beu 94] (from [BrA 97]).

is shown in Fig. 6.16, where only the $S_{11}$ resonance alone has been considered. The result using $W_{\gamma N}^{B S^{\prime}}$ is not shown because it is very similar to the one with $W_{\gamma N}^{B S}$. One readily notes considerable differences for the various prescriptions. The largest total cross section is obtained with the spectator on-shell, $W_{\gamma N}^{S}$, having its maximum at $750 \mathrm{MeV}$. If one puts the active nucleon on shell, i.e., takes $W_{\gamma N}^{N}$ or $W_{\gamma N}^{N^{\prime}}$ instead, the maximum is decreased by about $18 \%$ and slightly shifted towards higher energies. This decrease and shift can be understood as a result of the assignment of a higher invariant mass to the $\gamma N$ subsystem and the additional smearing due to the dependence on the angle between the spectator momentum and the photon, respective $\eta$ momentum (see Fig. 6.15) which leads to a larger effective width. The result is a slight upshift of the resonance position and a broadening, thus lowering of the maximum. One notes also only a little difference between putting the active nucleon before or after the interaction on-shell. From the foregoing discussion it is apparent that the curve for the Blankenbecler-Sugar assignment $W_{\gamma N}^{B S}$ is about halfway between the spectator on shell and the active nucleon on shell, because, according to Fig. $6.15, W_{\gamma N}^{B S}$ lies in between the spectator and active nucleon assignments.

In view of these results, one has to conclude that the choice for the invariant mass of the $\gamma N$ subsystem has a significant influence on the cross section. In order to obtain a cross section of the same magnitude for two different choices of $W_{\gamma N}$, one has to change the elementary helicity amplitude, too. This introduces a systematic uncertainty with respect to the determination of the elementary neutron amplitude, which can only be removed by a proper relativistic treatment.

\subsubsection{Final State Interaction in Incoherent Eta Photoproduction}

As already mentioned above, one major motivation for studying $\eta$ photoproduction on the deuteron is to obtain information on the elementary process on the neutron. This is of particular interest with respect to the isospin dependence of the production amplitude, i.e., 


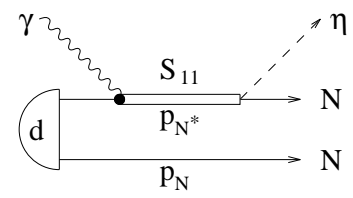

(a)

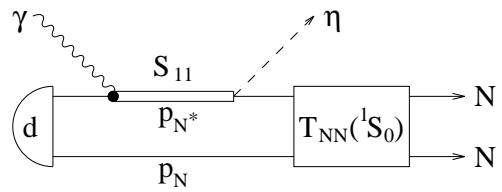

(b)

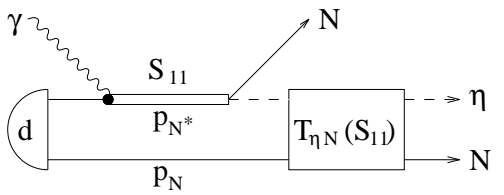

(c)

Fig. 6.17 Diagrammatic representation of the contributions to the $\gamma d \rightarrow \eta n p$ amplitude considered in [FiA 97]: (a) impulse approximation, (b) $N N$ rescattering and (c) $\eta N$ rescattering.

the relative size of its isoscalar $\left(t_{\gamma \eta}^{(s)}\right)$ to its isovector $\left(t_{\gamma \eta}^{(v)}\right)$ part. The method of extracting the neutron amplitude is based on the so-called spectator-nucleon model, in which the pure quasifree production is considered as the only mechanism for the knock-out reaction $d(\gamma, \eta N) N$. Therefore, a careful investigation of the validity of this approximation, i.e., the estimation of possible other competing effects is necessary. One can expect that such effects become important close to the reaction threshold. In this region, the smallness of the excitation energy in the final $n p$ system and the large momentum transfer (which is of the order of the $\eta$ mass in the $\gamma d$ c.m. frame) lead to a kinematical situation, where the two final nucleons move primarily together with a large total, but small relative momentum. For this kinematics, the spectator model is expected to give a very small cross section since the momenta of both nucleons are large and, on the other hand, the corrections due to the strong $N N$ interaction may be significant.

Recently, such final state interaction effects in the $\gamma d \rightarrow \eta n p$ process have been studied by Fix and myself [FiA 97]. As full amplitude we have included one-loop $N N$ and $\eta N$ rescattering terms as shown in Fig. 6.17 besides the pure impulse approximation (IA). The resulting total cross section is shown in Fig. 6.18. One readily notes, that the simple spectator approach cannot describe the experimental data close to the threshold (see also [SaF 95]). As has been mentioned above, at small photon energies, the $\gamma d \rightarrow \eta n p$ process is governed in the spectator model by the high Fourier components of the deuteron wave function, which have only a small probability. On the other hand, the final state interaction provides a mechanism for bypassing this suppression. Indeed, quite a significant contribution from $N N$ rescattering is found. It turns out to be even dominant in the vicinity of the threshold, and at $\omega=720$ $\mathrm{MeV}$ it still increases the IA result by about $10 \%$. The $\eta N$ interaction is relatively less important, but is also significant, mainly through the constructive interference between $N N$ and $\eta N$ rescattering contributions. With inclusion of both rescattering effects, we are able to reproduce the experimental cross section with a ratio of isoscalar to proton amplitude $\alpha=0.11$.

The influence of $N N$ rescattering on the $\eta$ momentum distribution at a fixed angle $\theta_{\eta}$ is shown in Fig. 6.19. As expected, the strong interaction between the final nucleons in the ${ }^{1} S_{0}$ state changes drastically the cross section for large $\eta$ momentum values. When $q$ reaches its maximum, the excitation energy $E_{n p}$ in the $n p$ pair becomes very small, and thus the resonant ${ }^{1} S_{0}$ state appears as a rather narrow peak. The same effect appears in charged 


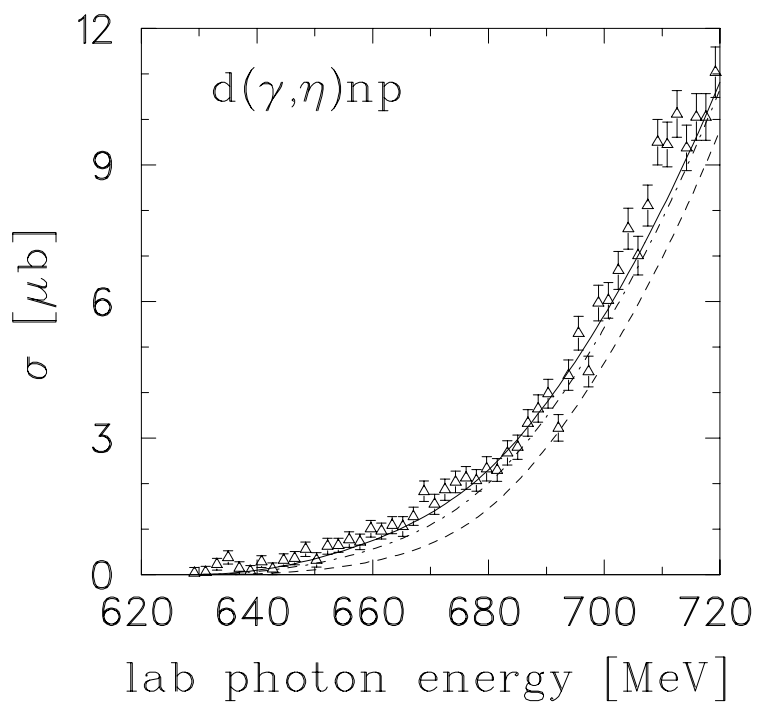

Fig. 6.18 Total $\gamma d \rightarrow \eta n p$ cross section compared to inclusive $\gamma d \rightarrow \eta X$ experimental data [KrA 95]. The full and dashed lines represent the results obtained with and without allowance for rescattering of the final particles, respectively. The dash-dotted line includes only IA and $N N$ rescattering (from [FiA 97]).
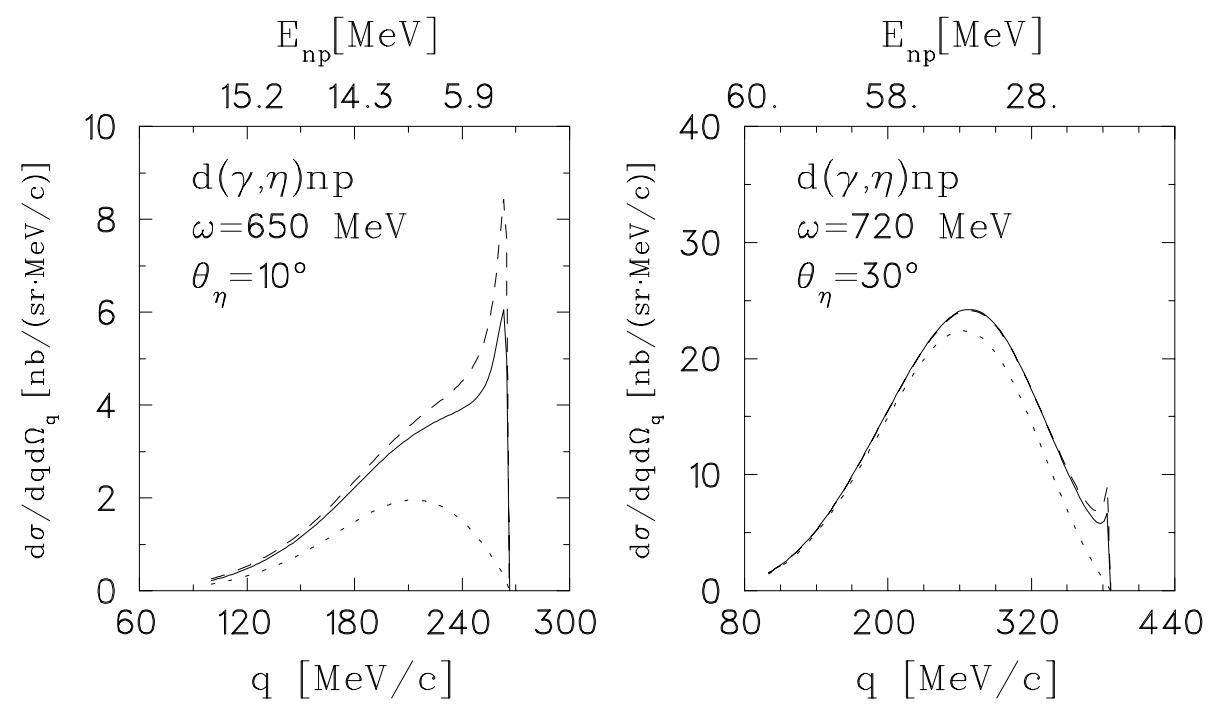

Fig. 6.19 The $\eta$ meson spectra at forward emission angles for $d(\gamma, \eta) n p$ at two different photon energies and angles. The dotted curves show the pure impulse approximation, whereas the full curves include the interaction between the outgoing nucleons. The dashed curves represent the results obtained without the $D$-wave contribution to the $N N$ rescattering amplitude. The excitation energy $E_{n p}$ in the final $N N$ system is indicated at the top abscissa (from [FiA 97]). 

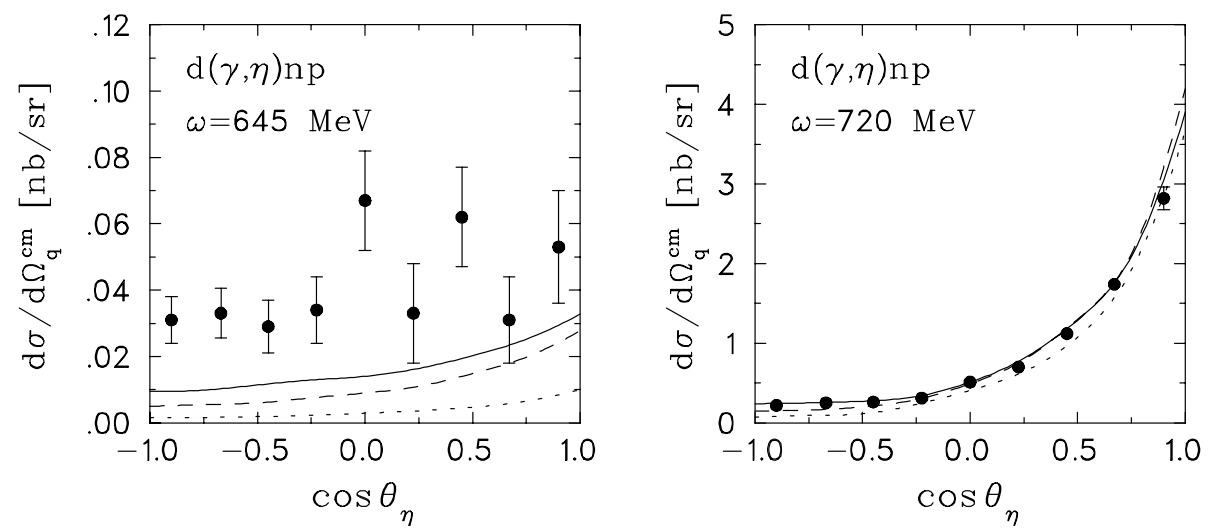

Fig. 6.20 The differential $d(\gamma, \eta) n p$ cross section, calculated in the $\gamma d$ c.m. frame (from [FiA 97]). Shown are the IA prediction (dotted lines), the successive addition of $N N$ (dashed lines) and $\eta N$ (full lines) rescattering. The experimental points represent the inclusive $\gamma d \rightarrow \eta X$ measurements from [KrA 95].

$\pi$ photo- and electroproduction on the deuteron [Lag 78, KoA 87] as well as in deuteron electrodisintegration [FaA 76]. In principle, the experimental observation of this peak in the high $\eta$ momentum spectrum may serve as another evidence for the isovector nature of the $S_{11}$ photoexcitation. In the hypothetical case that the isoscalar amplitude is much larger than the isovector one, the low-energy $N N$ rescattering would be dominated by the ${ }^{3} S_{1}$ state, which does not exhibit any resonant behaviour at $E_{n p} \approx 0$. In conclusion, one sees that the role of $N N$ rescattering is quite important, especially in the threshold region. At higher energies, the main part of the cross section is dominated by the IA, which gives a rather broad quasifree bump, where the role of $N N$ rescattering is expected to be of minor importance.

The effect of $\eta N$ rescattering is demonstrated in Fig. 6.20 for the $\eta$ angular distribution in the $\gamma d$ c.m. frame, where the theory is compared with the experimental data for the inclusive reaction $\gamma d \rightarrow \eta X$ [KrA 95]. In view of the small isoscalar part $t_{\gamma \eta}^{(s)}$ of the elementary amplitude and the large momentum mismatch, the contribution from the coherent $\gamma d \rightarrow \eta d$ process is expected to be negligible and, thus, the inclusive $\eta$ spectrum is dominated by the deuteron break-up channel. In the backward direction, the increase from $\eta N$ rescattering is in part kinematically enhanced. At these angles, the nucleons leave the interaction region with large momenta. Therefore, the spectator model gives a very small cross section underestimating the data by roughly a factor of 3 for $\omega=720 \mathrm{MeV}$. In this situation, the $\eta N$ rescattering mechanism, allowing the large transferred momentum to be shared between the two participating nucleons, becomes much more effective. The resonant character of the $\eta N$ interaction appears more pronounced at forward angles. Although its strong inelasticity decreases the cross section at high photon energies, close to threshold this effect is more than compensated by the attraction in the $\eta N$ system.

\subsection{The Gerasimov-Drell-Hearn Sum Rule}

The Gerasimov-Drell-Hearn (GDH) sum rule connects the anomalous magnetic moment of a particle with the energy weighted integral - henceforth denoted by $I^{G D H}(\infty)$ - from threshold up to infinity over the spin asymmetry of the total photoabsorption cross section, i.e., the difference of the total photoabsorption cross sections for circularly polarized photons on a target with spin parallel and antiparallel to the spin of the photon. In detail it reads for a 
particle of mass $M$, charge $e Q$, anomalous magnetic moment $\kappa$ and spin $S$

$$
I^{G D H}(\infty)=\int_{0}^{\infty} \frac{d k}{k}\left(\sigma^{P}(k)-\sigma^{A}(k)\right)=4 \pi^{2} \kappa^{2} \frac{e^{2}}{m^{2}} S,
$$

where $\sigma^{P / A}(k)$ denote the total absorption cross sections for circularly polarized photons on a target with spin parallel and antiparallel to the photon spin, respectively, and the anomalous magnetic moment is defined by the total magnetic moment operator of the particle

$$
\vec{M}=(Q+\kappa) \frac{e}{m} \vec{S} .
$$

This sum rule gives a very interesting relation between a magnetic ground state property of a particle and an integral property of its whole excitation spectrum. In other words, this sum rule shows that the existence of a nonvanishing anomalous magnetic moment points directly to an internal dynamic structure of the particle. Furthermore, because the rhs of (6.2) is positive, it tells us that the integrated, energy-weighted total absorption cross section of a circularly polarized photon on a particle with its spin parallel to the photon spin is bigger than the one on a target with its spin antiparallel, if the particle possesses a nonvanishing anomalous magnetic moment. The recent interest in this sum rule stems from the study of the spin dependent structure functions in deep inelastic scattering [Dre 95].

The GDH sum rule has first been derived by Gerasimov [Ger 65] and, shortly afterwards, independently by Drell and Hearn [DrH 66]. It is based on two ingredients which follow from the general principles of Lorentz and gauge invariance, unitarity, crossing symmetry and causality of the forward Compton scattering amplitude of a particle. The first one is the low energy theorem of Low [Low 54] and Gell-Mann and Goldberger [GeG 54] for a spin one-half particle which later has been generalized to arbitrary spin [LaC 60, Sai 69, Fri 77]. The low energy theorem for the forward amplitude for elastic scattering of a photon with momentum $\vec{k}$ on a target with spin $\vec{S}$ and an anomalous magnetic moment $\kappa$ reads

$$
T_{\lambda \lambda}(\vec{k}, \vec{S})=-e^{2} \frac{Q^{2}}{m}+\lambda \kappa^{2} \frac{e^{2}}{m^{2}}\langle\mathbf{S} \cdot \mathbf{k}\rangle+\mathcal{O}\left(\mathbf{k}^{2}\right),
$$

where the first term describes the classical Thomson amplitude. It is important to note that the spin term is already of relativistic order.

The second ingredient is the assumption of an unsubtracted dispersion relation for the difference of the elastic forward scattering amplitudes for circularly polarized photons and a completely polarized target with spin parallel $\left(S_{P}\right)$ and antiparallel $\left(S_{A}\right)$ to the photon spin

$$
\Re e f(k)=\frac{\mathcal{P}}{\pi} \int_{-\infty}^{\infty} d k^{\prime} \frac{\Im m f(k)}{k^{\prime}-k},
$$

where

$$
\begin{aligned}
f(k) & =T_{\lambda \lambda}\left(k, S_{P}\right)-T_{\lambda \lambda}\left(k, S_{A}\right) \\
& =2 \kappa^{2} \frac{e^{2}}{m^{2}} k S+\mathcal{O}\left(k^{2}\right) .
\end{aligned}
$$

Certainly, this assumption appears to be the weakest point on which the GDH sum rule is based. A hand waving argument in support of it is, that with increasing energy, due to the increased phase space and increased number of produced particles, the dependence of the total cross section on the target spin orientation will decrease, so that the spin asymmetry converges more rapidly than the unpolarized cross section.

Using crossing symmetry and the optical theorem, one obtains then

$$
\Re e f(k)=\frac{k}{2 \pi^{2}} \mathcal{P} \int_{0}^{\infty} d k^{\prime} k^{\prime} \frac{\sigma^{P}\left(k^{\prime}\right)-\sigma^{A}\left(k^{\prime}\right)}{k^{\prime 2}-k^{2}} .
$$

Finally, the derivative at $k=0$ yields the GDH sum rule. 

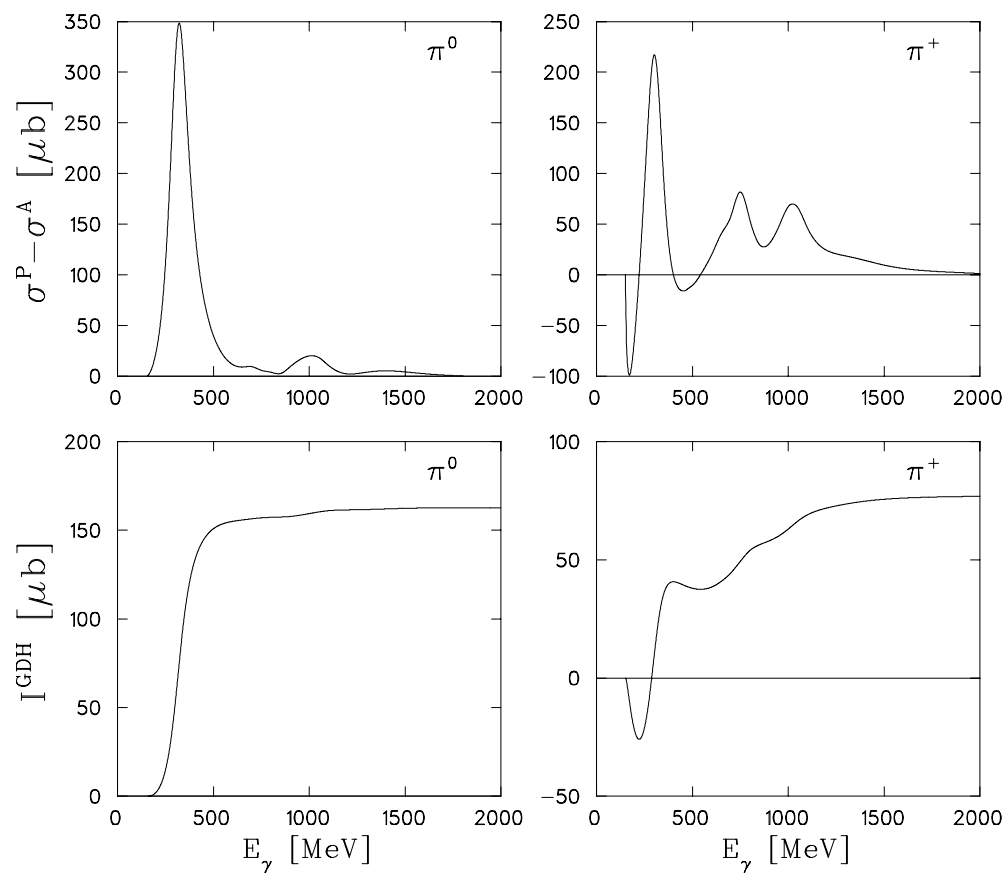

Fig. 6.21 The spin asymmetry $\sigma^{P}-\sigma^{A}$ and the finite GDH integral as function of the upper integration energy for the proton from the VPI multipole analysis (fit SM95) [SAID] (from [ArK 97]).

\subsubsection{The GDH Sum Rule for the Nucleon}

I will first consider the GDH sum rule for the nucleon. Since proton and neutron have large anomalous magnetic moments, one finds correspondingly large GDH sume rule predictions for them, i.e.,

$$
I_{p}^{G D H}(\infty)=204.8 \mu \mathrm{b}, \quad \text { and } \quad I_{n}^{G D H}(\infty)=233.2 \mu \mathrm{b}
$$

Although this sum rule is known for more than 30 years, it has never been evaluated by a direct integration of experimental data on $\sigma^{P}\left(k^{\prime}\right)-\sigma^{A}\left(k^{\prime}\right)$. The absorptive processes to be included are for the proton (analogously for the neutron)

$$
\begin{aligned}
& \gamma+p \rightarrow p+\pi^{0}, \\
& \gamma+p \rightarrow n+\pi^{+}, \\
& \gamma+p \rightarrow N+\pi+\pi, \text { etc. }
\end{aligned}
$$

Early evaluation by Karliner [Kar 73] of the finite GDH integral

$$
I^{G D H}(k)=\int_{0}^{k} \frac{d k^{\prime}}{k^{\prime}}\left(\sigma^{P}\left(k^{\prime}\right)-\sigma^{A}\left(k^{\prime}\right)\right)
$$

based on a multipole analysis of experimental data on single pion photoproduction on the nucleon, did not give conclusive results due to the lack of data at higher energies, and even present day data do not allow a definite answer as to its validity (see e.g. [SaW 94]). The contribution to the GDH sum rule for the proton from single pion production is shown in Fig. 6.21. Explicit integration up to $2 \mathrm{GeV}$ gives the values [ArK 97] (see also [SaW 94])

$$
I_{p}^{G D H}(2 \mathrm{GeV})_{\pi}=239 \mu \mathrm{b} \quad \text { and } \quad I_{n}^{G D H}(2 \mathrm{GeV})_{\pi}=168 \mu \mathrm{b} .
$$


Furthermore, an estimation of the $2 \pi$ contribution up to $1.7 \mathrm{GeV}$ from Karliner [Kar 73]

$$
I_{p}^{G D H}(1.7 \mathrm{GeV})_{\pi \pi}=65 \mu \mathrm{b} \quad \text { and } \quad I_{n}^{G D H}(1.7 \mathrm{GeV})_{\pi \pi}=35 \mu \mathrm{b}
$$

worsens the comparison with the sum rule values.

\subsubsection{The GDH Sum Rule for the Deuteron}

Applying the GDH sume rule to the deuteron, one finds a very interesting feature. On the one hand, the deuteron has isospin zero, ruling out the contribution of the large nucleon isovector anomalous magnetic moments to its magnetic moment, and, therefore, only a very small anomalous magnetic moment is expected. In fact, the experimental value is $\kappa_{d}=-0.143$ resulting in a GDH prediction of $I_{d}^{G D H}(\infty)=0.65 \mu \mathrm{b}$, which is more than two orders of magnitude smaller than the nucleon values. On the other hand, considering the possible absorptive processes, (i) photodisintegration $\gamma+d \rightarrow n+p$, (ii) single pion production, coherent $\gamma+d \rightarrow d+\pi^{0}$ and incoherent $\gamma+d \rightarrow N+N+\pi$, (iii) two pion production etc., one notes first, that the incoherent pion production on the deuteron is dominated by the quasifree production on the nucleons bound in the deuteron. Thus it is plausible to expect from these processes a contribution to the GDH sum rule roughly given by the sum of the proton and neutron $\mathrm{GDH}$ values, i.e., $438 \mu \mathrm{b}$. Additional contributions arise from the coherent $\pi^{0}$ production channel. In order to obtain the small total deuteron GDH sum rule, one, therefore, needs a large negative contribution of about the same size for cancellation. Indeed, one has an additional channel not present for the nucleon, namely the photodisintegration channel which is the only photoabsorption process below the pion production threshold. A closer look shows in fact that at very low energies near threshold a sizable negative contribution arises from the $M 1$ transition to the resonant ${ }^{1} S_{0}$ state, because this state can only be reached if the spins of photon and deuteron are antiparallel, and is forbidden for the parallel situation as has been pointed out, for example in [BaD 67].

Recently, we have evaluated explicitly the GDH sum rule for the deuteron by integrating the difference of the two total photoabsorption cross sections with photon and deuteron spins parallel and antiparallel up to a photon energy of $550 \mathrm{MeV}$ [ArK 97]. Three contributions have been included: (i) the photodisintegration channel $\gamma d \rightarrow n p$, (ii) the coherent pion production $\gamma d \rightarrow \pi^{0} d$, and (iii) the incoherent pion production $\gamma d \rightarrow \pi N N$. As already mentioned, the upper integration limit of $550 \mathrm{MeV}$ has been chosen because on the one hand one finds sufficient convergence for the photodisintegration channel, while on the other hand, only single pion photoproduction has been considered, thus limiting the applicability of the present theoretical treatment to energies not too far above the two pion production threshold as long as significant contributions from multipion production

cannot be expected. Indeed, the recent evaluation of $I^{G D H}$ using experimental data for the nucleon by Sandorfi et al. [SaW 94] indicates that significant contributions from two-pion production start only above this energy. I will now discuss the three contributions separately.

\section{(i) GDH contribution from photodisintegration}

At low energies one has dominant contributions from the $E 1$ and $M 1$ multipoles. However, the $E 1$ transitions cancel each other almost completely. Thus, at low energies remain the $M 1$ transitions, essentially to ${ }^{1} S_{0}$ and ${ }^{3} S_{1}$ states. Of these, the ${ }^{1} S_{0}$ contribution is dominant because of the large isovector part of the $M 1$ operator coming from the large isovector anomalous magnetic moment of the nucleon. It is particularly strong close to break-up threshold, where the ${ }^{1} S_{0}$ state is resonant, and can only be reached by the antiparallel spin combination 
resulting in a strong negative contribution to the GDH sum rule. The results are summarized in Fig. 6.22, where the cross section difference and the GDH integral is shown. The GDH values are listed in Tab. 6.1.
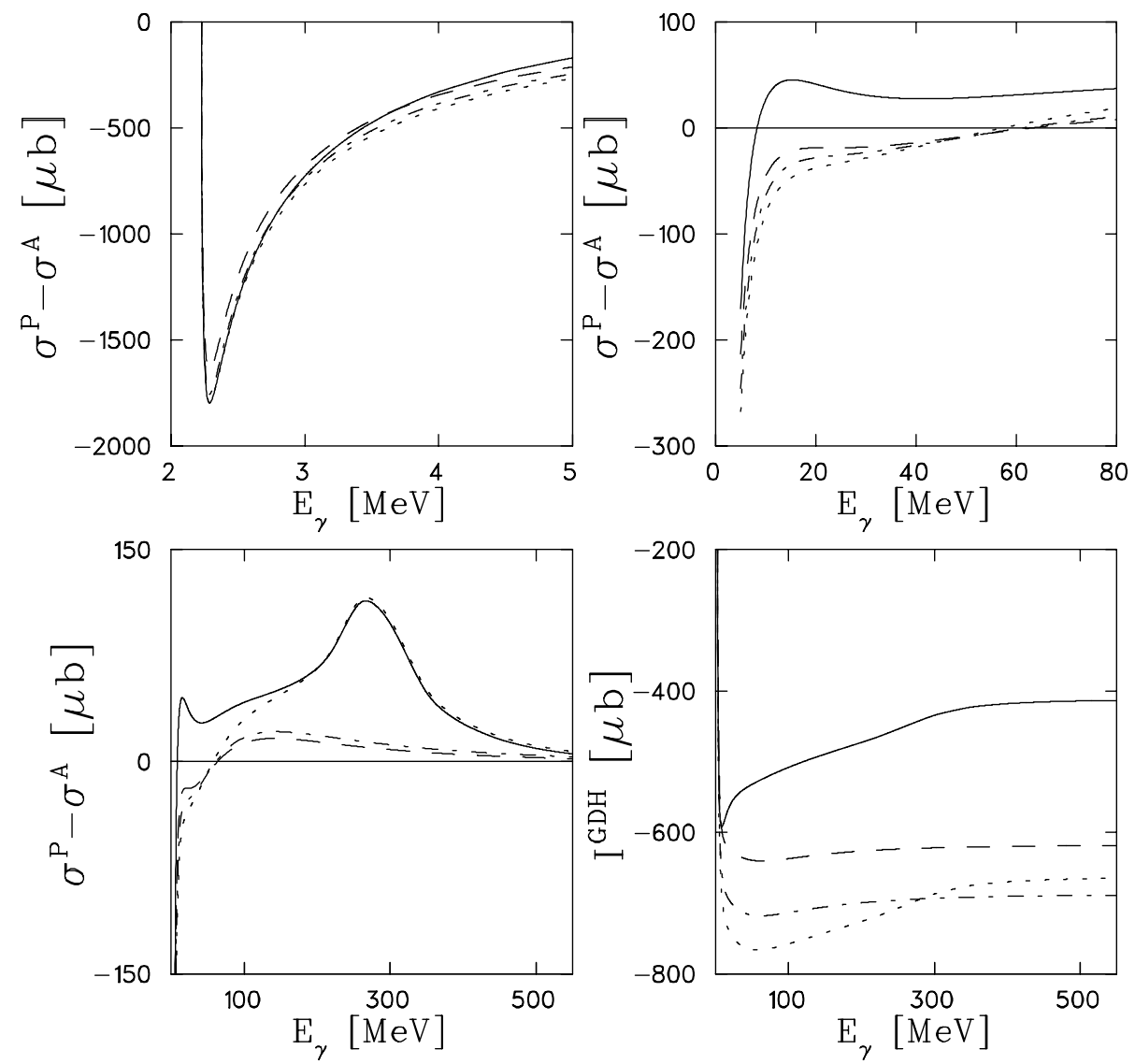

Fig. 6.22 Contribution of the photodisintegration channel to the GDH sum rule for the deuteron. Two upper and lower left panels: difference of the cross sections in various energy regions; lower right panel: $I_{\gamma d \rightarrow n p}^{G D H}$ as function of the upper integration energy. Dashed curves: normal (N), dash-dot: N+MEC, dotted: N+MEC+IC, and full curves $\mathrm{N}+\mathrm{MEC}+\mathrm{IC}+\mathrm{RC}$ (from [ArK 97]).

Table 6.1 Various contributions of the photodisintegration channel to the GDH integral for the deuteron integrated up to $550 \mathrm{MeV}$ in $\mu \mathrm{b}$.

\begin{tabular}{|ccc|c|}
\hline $\mathrm{N}$ & $\mathrm{N}+\mathrm{MEC}$ & $\mathrm{N}+\mathrm{MEC}+\mathrm{IC}$ & $\mathrm{N}+\mathrm{MEC}+\mathrm{IC}+\mathrm{RC}$ \\
\hline-619 & -689 & -665 & -413 \\
\hline
\end{tabular}

One readily notes the huge negative contribution from the ${ }^{1} S_{0}$ state at low energies (see the upper left panel of Fig. 6.22). It is the result of the large isovector anomalous magnetic moment of the nucleon. Indeed, for a vanishing anomalous nucleon magnetic moment one finds $I_{d}^{G D H}(550 \mathrm{MeV})_{\kappa_{N}=0}=7.3 \mu \mathrm{b}$. The effects from MEC are relatively strong, resulting in an enhancement of the negative value by about 15 percent. It corresponds to the well-known 10 percent enhancement of the radiative capture of thermal neutrons on protons. Isobar effects are significant in the region of the $\Delta$ resonance, as expected. They give a positive contribution, but considerably smaller in absolute size than MEC. The largest 
positive contribution stems from relativistic effects $(\mathrm{RC})$ in the energy region up to about $100 \mathrm{MeV}$ (see the upper right panel of Fig. 6.22) reducing the GDH value in absolute size by more than 30 percent. This strong influence of relativistic effects is not surprising in view of the fact, that the correct form of the term linear in the photon momentum of the low energy expansion of the forward Compton scattering amplitude is only obtained if leading order relativistic contributions are included [Fri 77].

\section{(ii) GDH contribution from coherent pion production}

As contributions from single pion production one has to consider coherent and incoherent processes. The theoretical model used to calculate the contribution of the coherent pion production channel has been described above in Sect. 6.2. The reaction is clearly dominated by the magnetic dipole excitation of the $\Delta$ resonance from which one expects a strong positive $I_{\gamma d \rightarrow d \pi^{0}}^{G D H}$ contribution. The reason for this is that the $\Delta$ excitation is favoured if photon and nucleon spins are parallel compared to the antiparallel situation. Fig. 6.23 shows the result of our calculation. One sees the strong positive contribution from the $\Delta$ excitation giving a value of $I_{\gamma d \rightarrow d \pi^{0}}^{G D H}(550 \mathrm{MeV})=63 \mu \mathrm{b}$. The comparison with the unpolarized cross section, also plotted in Fig. 6.23, demonstrates the dominance of $\sigma^{P}$ over $\sigma^{A}$.
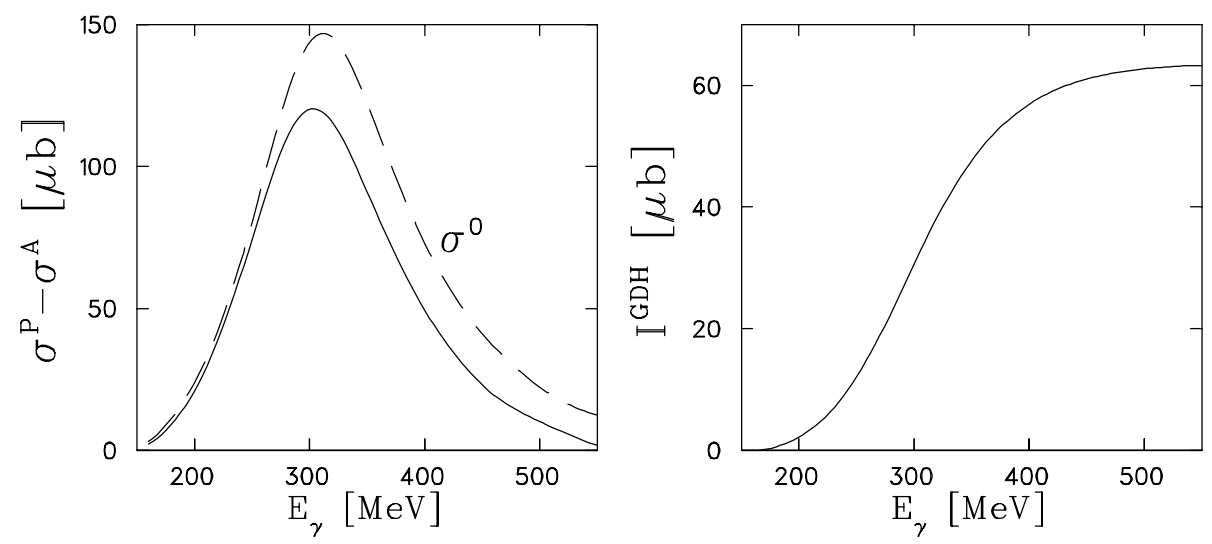

Fig. 6.23 Contribution of coherent $\pi^{0}$ production to the GDH sum rule for the deuteron. Left panel: difference of the cross sections (full curve), the dashed curve shows the unpolarized cross section; Right panel: $I_{\gamma d \rightarrow d \pi^{0}}^{G D H}$ as function of the upper integration energy (from [ArK 97]).

\section{(iii) GDH contribution from incoherent pion production}

The calculation of the incoherent $\gamma d \rightarrow \pi N N$ contributions to the GDH integral is based on the spectator-nucleon approach of Schmidt et al. [ScA 96]. In this framework, the reaction proceeds via the pion production on one nucleon while the other nucleon acts merely as a spectator. Thus, the $\gamma d \rightarrow \pi N N$ operator is given as the sum of the elementary $\gamma N \rightarrow \pi N$ operators of the two nucleons. The results are collected in Fig. 6.24. The upper part shows the individual contributions from the different charge states of the pion and their total sum to the spin asymmetry for pion photoproduction on both the deuteron and for comparison on the nucleon. One notes qualitatively a similar behaviour although the maxima and minima are smaller and also slightly shifted towards higher energies for the deuteron. In the lower part of Fig. 6.24 the corresponding GDH integrals are shown. A large positive contribution comes from $\pi^{0}$ production whereas the charged pions give a negative but - in absolute size - smaller contribution to the GDH value. Up to an energy of 550 

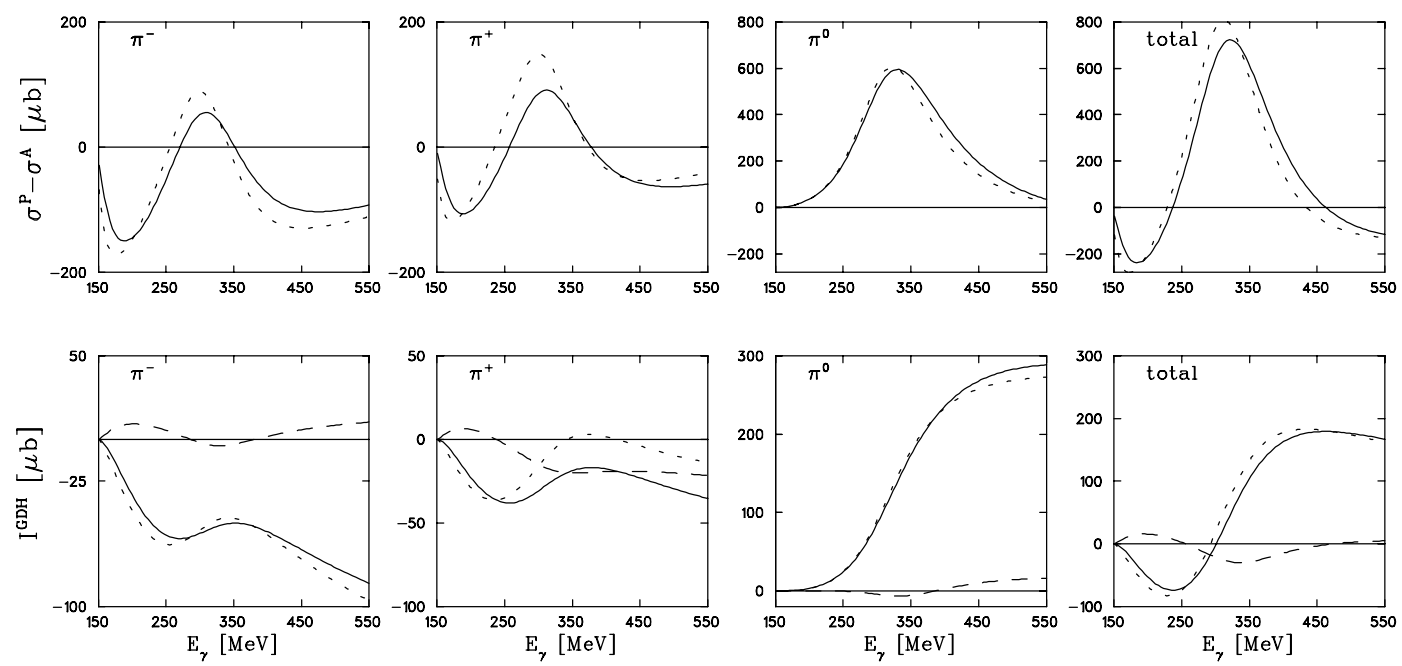

Fig. 6.24 Contribution of the incoherent $\pi$ production to the GDH sum rule for the deuteron and the nucleon. Upper part: difference of the cross sections; lower part: $I_{\gamma d \rightarrow N N \pi}^{G D H}$ as function of the upper integration limit, full curves for the deuteron, dotted curves for the nucleon. In the case of $\pi^{0}$ production, the dotted curve shows the summed proton and neutron contributions. The dashed curves show the appropriate differences $I_{\gamma d \rightarrow N N \pi}^{G D H}-I_{p}^{G D H}-I_{n}^{G D H}$ (from [ArK 97]).

$\mathrm{MeV}$, one finds for the total contribution of the incoherent pion production channels a value $I_{\gamma d \rightarrow N N \pi}^{G D H}(550 \mathrm{MeV})=167 \mu \mathrm{b}$, which is remarkably close to the sum of the neutron and proton values for the given elementary model $I_{n}^{G D H}(550 \mathrm{MeV})+I_{p}^{G D H}(550 \mathrm{MeV})=163 \mu \mathrm{b}$. This fact is also indicated by the dashed curves in the lower part of Fig. 6.24 which represent the appropriate differences of $I_{\gamma d \rightarrow N N \pi}^{G D H}-I_{p}^{G D H}-I_{n}^{G D H}$. It underlines again that the total cross section is dominated by the quasifree process. However, as is evident from Fig. 6.24, convergence is certainly not reached at this energy. Furthermore, the elementary pion production operator used in [ScA 96] had been constructed primarily to give a realistic description of the $\Delta$ resonance region. In fact, it underestimates the GDH inegral up to 550 $\mathrm{MeV}$ by about a factor two compared to a corresponding evaluation based on a multipole analysis of experimental pion photoproduction data. For this reason we cannot expect that this model gives also a good description of experimental data above $400 \mathrm{MeV}$. However, the important result is, that the total GDH contribution from the incoherent process is very close to the sum of the free proton and neutron GDH integrals, which we expect to remain valid for an improved elementary production operator.

\section{(iv) Total GDH contribution for the deuteron}

The contributions from all three channels and their sum are listed in Tab. 6.2. A very interesting and important result is the large negative contribution from the photodisintegration channel near and not too far above the break-up threshold with surprisingly large relativistic effects below $100 \mathrm{MeV}$. Hopefully, this low energy feature of the GDH sum rule could be checked experimentally in the near future. For the total GDH value from explicit integration up to $550 \mathrm{MeV}$, we find a negative value $I_{d}^{G D H}(550 \mathrm{MeV})=-183 \mu \mathrm{b}$. However, as we have mentioned above, some larger uncertainty lies in the contribution of the incoherent pion production channel because of shortcomings of the model of the elementary production amplitude above the $\Delta$ resonance. If we use instead of the model value $I_{\gamma d \rightarrow N N \pi}^{G D H}(550 \mathrm{MeV})=167 \mu \mathrm{b}$ 
Table 6.2 Contributions of the different absorption channels to the GDH integral for the deuteron integrated up to $550 \mathrm{MeV}$ in $\mu \mathrm{b}$.

\begin{tabular}{|ccccc|c|}
\hline$\gamma d \rightarrow n p$ & $\gamma d \rightarrow d \pi^{0}$ & $\gamma d \rightarrow n p \pi^{0}$ & $\gamma d \rightarrow n n \pi^{+}$ & $\gamma d \rightarrow p p \pi^{-}$ & total \\
\hline-413 & 63 & 288 & -35 & -86 & -183 \\
\hline
\end{tabular}

(cf. previous section) the sum of the GDH values of neutron and proton by integrating the cross section difference, obtained from a multipole analysis of experimental data (fit SM95 from [SAID]), giving $I_{n}^{G D H}(550 \mathrm{MeV})+I_{p}^{G D H}(550 \mathrm{MeV})=331 \mu \mathrm{b}$, we find for the deuteron $I_{d}^{G D H}(550 \mathrm{MeV})=-19 \mu \mathrm{b}$, which we consider a more realistic estimate. Since this value is still negative, a positive contribution of about the same size should come from contributions at higher energies in order to fulfil the small GDH sum rule for the deuteron, provided that the sum rule is valid. These contributions should come from the incoherent single pion production above $550 \mathrm{MeV}$ because here convergence had not been reached in contrast to the other two channels, photodisintegration and coherent pion production, and in addition, from multipion production.

The foregoing results show that the spin asymmetry and the GDH sum rule of the deuteron are very interesting observables because of a strong anticorrelation of photodisintegration and pion production. Further improvements are necessary with respect to the elementary single and double pion production and the treatment of photodisintegration above the $\Delta$ resonance.

\subsection{Parity Violation in Quasifree Electron Scattering}

The recent interest in studying parity violation in electron scattering by electroweak interference is motivated by the possibility to investigate the so-called strangeness or better $s \bar{s}$ content of the nucleon, a quantity of particular interest with respect to the nucleon spin structure functions as measured in deep inelastic scattering [AsB 93, AbA 95, AdA 94]. In fact, several experiments to measure parity violation in electron scattering off hydrogen and deuterium are presently underway [BeM 90, FiS 91, Har 93, BeA 96]. In these experiments, deuterium serves as a neutron target and for this reason quasifree kinematics is preferred in order to minimize possible interaction effects.

Parity violation in inelastic electron deuteron scattering has been studied theoretically by Hwang et al. [HwH 80, HwH 81] in the low energy and momentum transfer domain, by Hadjimichael et al. [HaP 92] for a larger kinematical range, in particular for quasifree kinematics at high momentum transfers, and very recently by Mosconi and Ricci [MoR 97]. Relativistic contributions have been considered by Poulis [Pou 96a, Pou 96b]. While in [HwH 81] the parity violation by electroweak interference as well as through parity violating nuclear components has been considered, the latter has been neglected throughout in the more recent evaluations [HaP 92, Pou 96a, Pou 96b, MoR 97]. Already in [HaP 92] it has been remarked that such effects should be investigated in order to see for which kinematical situations they can indeed be neglected or whether they have to be included. This has been done in a recent work by Küster and myself [KuA 97].

The presence of a parity violating $N N$ potential $V^{p n c}$ has the consequence that the nuclear states are no longer states of good parity, i.e., besides a dominant state $\left|J^{\pi}\right\rangle$ of angular momentum $J$ and parity $\pi$, there will be a small admixture of opposite parity $\left|J^{-\pi}\right\rangle$, with an amplitude $\mathcal{F}$ of the order $10^{-6}$ [McK 69]. In the case of the deuteron, the admixture of opposite parity (pnc) components must result in additional small $P$ waves. For the calculation 

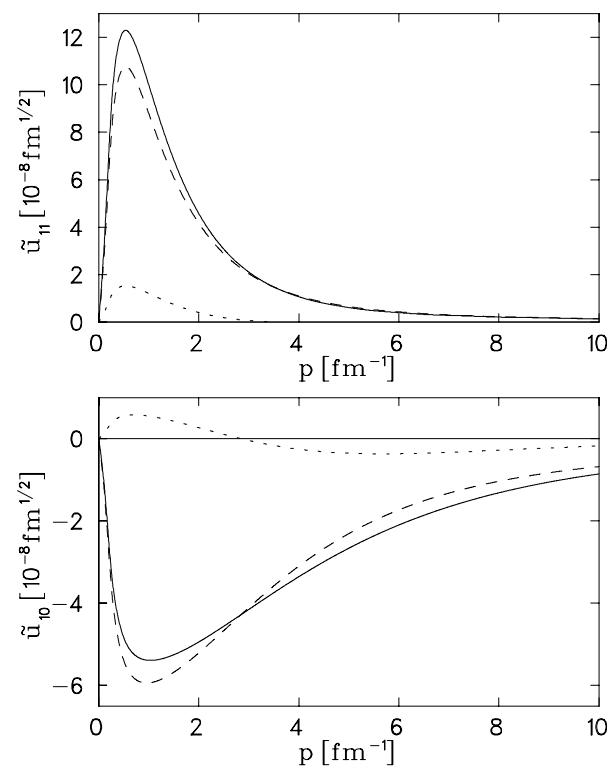

Fig. 6.25 Radial parts of pnc $P$ wave components (full) of the deuteron in momentum space. Also shown are the contributions generated separately from $S$ (dashed) and $D$ (dotted) components (from [KuA 97]).

of these $P$ wave components, the one-boson-exchange model of Desplanques, Donoghue and Holstein [DeD 80] has been used for $V^{p n c}$. Based on the quark model and $S U(6)_{w}$ symmetry, they made predictions for all meson-nucleon couplings from both charged and neutral current pieces of the weak Hamiltonian.

In order to calculate the opposite parity admixture of the deuteron wave function, firstorder perturbation theory is sufficient. The radial functions of the pnc components are shown in Fig. 6.25. For the unperturbed wave function, the parametrization by Machleidt et al. [MaH 87, Mac 89] for the Bonn OBEPQ model has been used. In momentum space, the parity violating $P$ waves are much more spread out than the normal $S$ and $D$ waves, indicating a much shorter range in coordinate space. This is particularly pronounced in the ${ }^{1} P_{1}$-part, because the $\pi$ exchange part of $V^{p n c}$ does not contribute here. The total $P$ state probability is $P_{P}=2.7 \times 10^{-14}$, which corresponds to an admixture amplitude that is of the order of magnitude expected [McK 69] and in qualitative agreement with [HwH 81]. In principle, parity violating components will also appear in the final scattering states. However, one expects a negligible effect for quasifree kinematics.

The resulting longitudinal beam asymmetries $\mathcal{A}^{\gamma_{a}}$ and $\mathcal{A}^{Z}$ (see (5.30) and (5.31) for their definition) are shown in Fig. 6.26. Since for a given momentum transfer $q^{l a b}$ the asymmetry depends also on the electron kinematics through the lepton density matrices, we have chosen two laboratory scattering angles, one at a more forward direction $\left(\theta_{e}=35^{\circ}\right)$ and one backward angle $\left(\theta_{e}=170^{\circ}\right)$. One readily notes that the beam asymmetry $\mathcal{A}^{\gamma_{a}}$ due to the $P$ wave in the deuteron varies strongly with the scattering angle. Indeed, it is relatively more suppressed for backward scattering than $\mathcal{A}^{Z}$. Furthermore, it is apparent that $\mathcal{A}^{\gamma_{a}}$ is negligible compared to $\mathcal{A}^{Z}$ over the whole range of momentum transfers considered here. For the beam asymmetry due to electroweak interference, the dominant contribution comes from the term proportional to $a_{a} F_{T}^{Z_{v}}$, but the dependence on the electron scattering angle is mainly a result of the term proportional to $a_{a} F_{L}^{Z_{v}}$. In order to compare our results on the asymmetry $\mathcal{A}^{Z}$ from electroweak interference with those reported in [HaP 92], we show in Fig. 6.27 the asymmetries on a logarithmic scale. For $\mathcal{A}^{Z}$ we find very good agreement with their results 

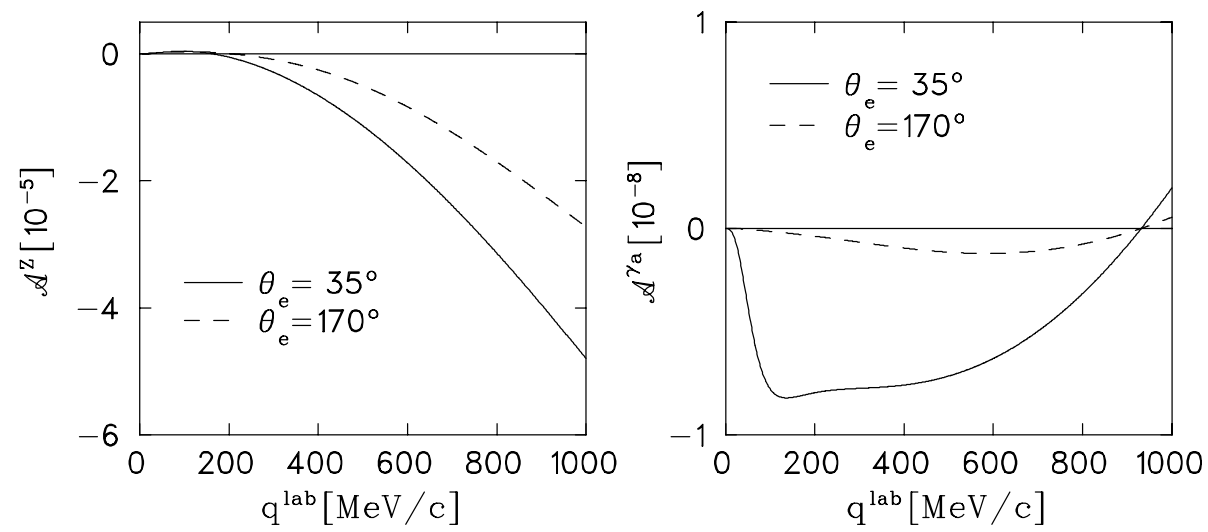

Fig. 6.26 Longitudinal asymmetry for polarized electrons along the quasifree ridge from electroweak interference (left panel) and from the pnc deuteron components (right panel) for forward and backward electron scattering $\left(\theta_{e}=35^{\circ}\right.$ and $\left.170^{\circ}\right)$ in the laboratory frame (from [KuA 97]).

for the quasifree case in plane wave Born approximation.
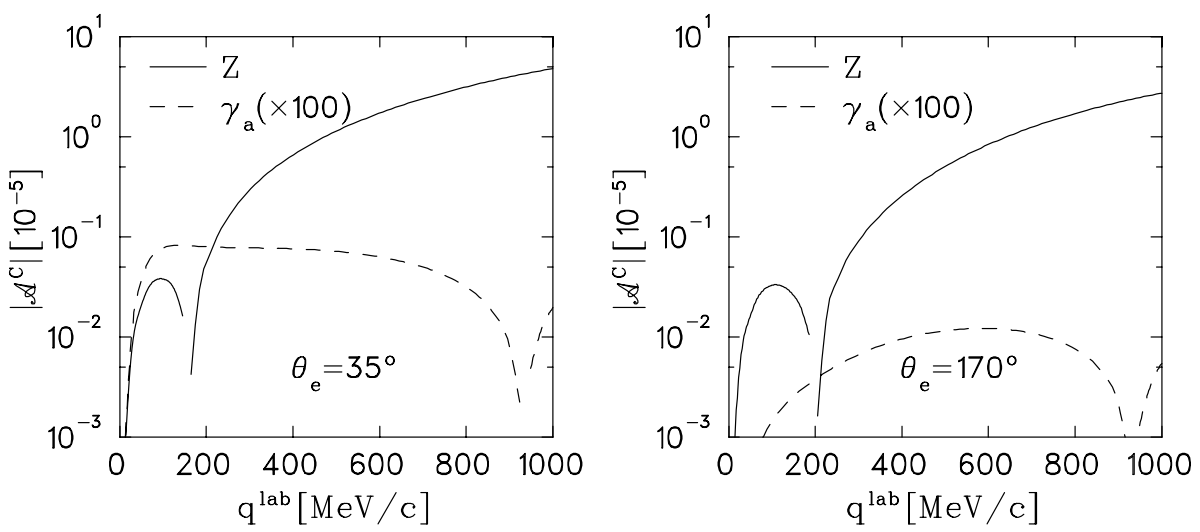

Fig. 6.27 As Fig. 6.26 but on a logarithmic scale (from [KuA 97]).

As already mentioned, there is a great deal of interest in experiments to determine strange quark contributions to hadronic matrix elements. In connection with this study, the SAMPLE experiment at MIT-Bates [BeA 96] is of special interest, since it measures the strange magnetic form factor $G_{M}^{(s)}$ at quite low momentum transfer as determined in parity violating electron scattering off hydrogen and deuterium. Even here, we have not found significant effects from parity violation in the hadronic sector.

In order to study parity violation which originates from the hadronic sector in electromagnetic deuteron break-up, one has to go away from the quasifree kinematics to lower momentum transfer where it may become more comparable in size to the contribution from electroweak interference. But then one has to consider also pnc components in the final state and the contribution from meson exchange currents. In this respect, the present study should be considered as a starting point for further investigations, in particular with respect to the role of final state interaction, meson exchange currents and isobar configurations. 


\section{Conclusions and Outlook}

I hope these lectures have demonstrated convincingly, that the electroweak probe is still an interesting, very important and versatile tool, allowing one to reveal the internal structure of hadrons in great detail. Although a lot of insight has been gained from the past experimental and theoretical work, there still exist largely unexplored regions waiting to be unveiled. In the more distant past, mostly inclusive reactions had been studied due to the low duty cycle of the at that time existing electron machines. Only during the last decade the new generation of high duty cycle machines became available and allows one now to exploit the full power of the electroweak probe. Thus it is clear, that the thrust of present and future experimental research will lie on the study of more and more exclusive reactions.

In particular, the recent developments with respect to more intense and highly polarized beams and targets of higher density and polarization, and the availability of highly efficient polarimeters for the analysis of the final reaction products will open up a new era of studying polarization observables in much greater detail than could be done before, and thus will give us even more detailed information on the properties of hadrons. Because polarization observables in general constitute much more stringent tests for theoretical models, being more sensitive to small, but interesting amplitudes. To this end, we need further developments of even more intense polarized beams. Similarly, highly polarized targets are desirable, which can resist high intensity beams. Furthermore, the efficiency of polarimeters, for example, neutron polarimeters, needs to be pushed to higher values.

On the theory side, the role of effective degrees of freedom in nuclei in terms of nucleon, meson and isobar degrees of freedom and their relation to the underlying more fundamental quark-gluon degrees of freedom of QCD should be further clarified. Specifically, it will be extremely important to understand, how far in energy and momentum transfer we can push this framework of effective nucleon, meson and isobar degrees of freedom and whether signatures of explicit quark-gluon effects will be manifest in nuclei under certain kinematic conditions. Very likely, we will not find a clear cut borderline between the perturbative and nonperturbative regimes of QCD and it will be difficult to pin down genuine quark-gluon effects in view of the partly phenomenological ingredients of present theoretical models on both sides, effective nuclear d.o.f. and quark-gluon descriptions. Similarly, with respect to the internal nucleon, or in general, hadron structure, the nature of the effective constituent quark degrees of freedom should be revealed. In addition, in view of the increase of energy and momentum transfers involved in present and future experiments, the effects which arise from relativity should be considered with great care.

\section{Acknowledgement}

I would like to thank the organizers of this workshop, A.C. Fonseca, W. Plessas and P. Sauer, for giving me the opportunity to deliver these lectures. The collaboration of Jiři Adam, Elena Breitmoser, Alexander Fix, Gunilla Küster, Frank Ritz, Jr., Rüdiger Schmidt, Michael Schwamb, Thomas Wilbois, and Paul Wilhelm in various parts of the work reported here is gratefully acknowledged. Furthermore, I would like to thank Mariana Kirchbach for various helpful discussions and a critical reading of the manuscript. 


\section{References}

[AbA 95] K. Abe et al., Phys. Rev. Lett. 74, 346 (1995); 76, 25 (1995)

[AdA 94] D. Adams et al., Phys. Lett. B 336, 125 (1994); B 357, 248 (1995)

[AdA 96] J. Adam, Jr. and H. Arenhövel, Nucl. Phys. A 598, 462 (1996); A 614, 289 (1997)

[AdA 97] J. Adam, Jr. and H. Arenhövel, Nucl. Phys. A 614, 289 (1997)

[AdT 89] J. Adam, Jr., E. Truhlik, D. and Adamova, Nucl. Phys. A 492, 556 (1989)

[ArB 90] R.G. Arnold et al., Phys. Rev. C 42, R1 (1990)

[Are 80] H. Arenhövel, Z. Phys. A 297, 129 (1980)

[Are 81a] H. Arenhövel, Z. Phys. A 302, 25 (1981)

[Are 81b] H. Arenhövel, Lecture Notes in Physics, Vol. 137, 136 (1981)

[Are 94] H. Arenhövel, Lecture Notes in Physics, Vol. 426, 1 (1994)

[ArK 97] H. Arenhövel, G. Kreß, R. Schmidt, and P. Wilhelm, Phys. Lett. B 407, 1 (1997)

[ArS 91] H. Arenhövel and M. Sanzone, Few-Body Syst., Suppl. 3, 1 (1991)

[ArW 72] H. Arenhövel and H.J. Weber, Springer Tracts in Modern Physics 65, 58 (1972)

[AsB 93] J. Ashman et al., Z. Phys. C 57 (1993) 211

[AuC 85] S. Auffret et al., Phys. Rev. Lett. 55, 1362 (1985)

[BaD 67] G. Barton and N. Dombey, Phys. Rev. 162, 1520 (1967)

[BeA 96] E.J. Beise et al., Proc. 9th Amsterdam Miniconference "Electromagnetic Studies of the Deuteron", eds. B.L.G. Bakker, T. Ketel, and H. de Vries (NIKHEF, Amsterdam 1996) p. 112

[BeB 83] P. Becher, M. Böhm, and H. Joos, Eichtheorien der starken und elektromagnetischen Wechselwirkung (Teubner, Stuttgart 1983)

[BeJ 81] M. Bernheim et al., Phys. Rev. Lett. 46, 402 (1981)

[BeL 81] M. Betz and T.-S.H. Lee, Phys. Rev. C 23375 (1981)

[BeM 90] Proceedings Workshop on Parity Violation in Electron Scattering, eds. E.J. Beise and R.D. McKeown (World Scientific, Singapore 1990)

[Beu 94] W. Beulertz, PhD thesis, Bonn 1994.

[BeW 94]Ｇ. Beck, Th. Wilbois, and H. Arenhövel, Few-Body Syst. 17, 91 (1994)

[BlB 91] G.S. Blanpied et al., Phys. Rev. Lett. 67, 1206 (1991) 
[BlB 95] G. Blanpied et al, Phys. Rev. C 52, R455 (1995)

[BrA 97] E. Breitmoser and H. Arenhövel, Nucl. Phys. A 612, 321 (1997)

[CaM 82] A. Cambi, B. Mosconi, and P. Ricci, Phys. Rev. Lett. 48, 462 (1982).

[Dav 94] R. Davidson, Lecture Notes in Physics, Vol. 426, 61 (1994)

[DeD 80] B. Desplanques, J.F. Donoghue, and B.R. Holstein, Ann. Phys. (N.Y.) 124, 449 (1980)

[Dre 95] D. Drechsel, Prog. Part. Nucl. Phys., Vol. 34, 181 (1995)

[DrH 66] S.D. Drell and A.C. Hearn, Phys. Rev. Lett. 16, 908 (1966)

[ElF 88] Ch. Elster, W. Ferchländer, K. Holinde, D. Schütte, and R. Machleidt, Phys. Rev. C 37, 1647 (1988)

[FaA 76] W. Fabian and H. Arenhövel, Nucl. Phys. A 258, 461 (1976)

[FeG 58] R.P. Feynman and M. Gell-Mann, Phys. Rev. 109, 193 (1958)

[FiA 97] A. Fix and H. Arenhövel, Z. Phys. A 359, 427 (1997)

[FiS 91] J.M. Finn and P.A. Souder, Spokespersons, CEBAF-Proposal 91-010 (1991)

[Fri 76] J.L. Friar, Phys. Rev. Lett. 36, 510 (1976)

[Fri 77] J.L. Friar, Phys. Rev. C 16, 1504 (1977)

[GaH 76] M. Gari and H. Hyuga, Z. Phys. A 227, 291 (1976)

[GaH 81] M. Gari and H. Hebach, Phys. Rep. 72, 1 (1981)

[GaK 71] S. Galster, H. Klein, J. Moritz, K.H. Schmidt, D. Wegener, and J. Bleckwenn, Nucl. Phys. 32, 221 (1971)

[Ger 65] S.B. Gerasimov, Yad. Fiz. 2, 598 (1965) (Sov. J. Nucl. Phys. 2, 430 (1966))

[GeG 54] M. Gell-Mann and M.L. Goldberger, Phys. Rev. 96, 1433 (1954)

[Gre 76] A.M. Green, Rep. Prog. Phys. 39, 1109 (1976)

[GrS 79] A.M. Green and M. Sainio, J. Phys. G5, 503 (1979)

[HaH 89] W.C. Haxton, E.M. Henley, and M.J. Musolf, Phys. Rev. Lett. 63, 949 (1989)

[HaP 92] E. Hadjimichael, G.I. Poulis, and T.W. Donnelly, Phys. Rev. C 45, 2666 (1992)

[Har 93] D. von Harrach, Spokesperson, MAMI-Proposal A /1-93 (1993)

[HoP 76] G. Höhler et al., Nucl. Phys. B 114, 505 (1976)

[Hua 92] K. Huang, Quarks, Leptons and Gauge Fields (World Scientific, Singapore 1992)

[HwH 80] W.-Y.P. Hwang and E.M. Henley, Ann. Phys. (N.Y.) 129, 47 (1980) 
[HwH 81] W.-Y.P. Hwang, E.M. Henley, and G.A. Miller, Ann. Phys. (N.Y.) 137, 378 (1981)

[KaT 97] S.S. Kamalov, L. Tiator, and C. Bennhold, Phys. Rev. C 55, 98 (1997)

[Kar 73] I. Karliner, Phys. Rev. D 7, 2717 (1973)

[KoA 87] G. Köbschall et al., Nucl. Phys. A 466, 612 (1987)

[KrA 95] B. Krusche et al., Phys. Lett. B 358, 40 (1995)

[KuA 97]Ｇ. Küster and H. Arenhövel, Nucl. Phys. A 626, 911 (1997)

[LaC 60] L.I. Lapidus and Chou Kuang-Chao, Zh. Eksp. Teor. Fiz. 39, 1286 (1960) (Sov. JETP 12, 898 (1961))

[Lag 78] J.-M. Laget, Nucl. Phys. A 296, 388 (1978)

[LeA 87] W. Leidemann and H. Arenhövel, Nucl. Phys. A 465, 573 (1987)

[Lee 83] T.-S.H. Lee, Phys. Rev. Lett. 50, 1571 (1983); T.-S.H. Lee, Phys. Rev. C 29, 195 (1984)

[LeM 85] T.-S.H. Lee and A. Matsuyama, Phys. Rev. C 321986 (1985); Phys. Rev. C 36, 1459 (1987)

[LeY 56] T.D. Lee and C.N. Yang, Phys. Rev. 104, 254 (1956)

[Low 54] F.E. Low, Phys. Rev. 96, 1428 (1954)

[Mac 89] R. Machleidt, Adv. Nucl. Phys. Vol. 19, p. 189 (Plenum, New York, 1989)

[MaH 87] R. Machleidt, K. Holinde, and Ch. Elster, Phys. Rep. 149, 1 (1987)

[Mat 89] J.F. Mathiot, Phys. Rep. 173, 63 (1989)

[Mat 97] J.F. Mathiot, Lectures at this Workshop

[McK 69] B.H.J. McKellar, Proc. 3. Int. Conf. High Energy Phys. and Nucl. Structure, ed. S. Devons, Columbia University (1969) p. 682

[MoR 97] B. Mosconi and P. Ricci, Phys. Rev. C 55, 3115 (1997)

[MuD 94] M.J. Musolf, T.W. Donnelly, J. Dubach, S.J. Pollock, S. Kowalski, and E.J. Beise, Phys. Rep. 239, 1 (1994)

[Nac 86] O. Nachtmann, Phänomene und Konzepte der Elementarteilchenphysik (Vieweg, Braunschweig/Wiesbaden 1986)

[PDG 96] Particle Data Group, Phys. Rev. D 54, 1 (1996)

[PoS 87] H. Pöpping, P.U. Sauer, and X.-Z. Zhang, Nucl. Phys. A 474, 557 (1987)

[Pou 96a] G.I. Poulis, Proc. Int. Symp. High-energy Spin Physics (SPIN 96), eds. C.W. de Jager, T.J. Ketel, P.J. Mulders, J.E. Oberski, M. Oskam-Tamboezer, (World Scientific, 1997), hep-ph/9610536

[Pou 96b] G.I. Poulis, report ADP-96-47/T241, Few Body Syst. (in print) 
[RiA 97] F. Ritz, H. Arenhövel, and T. Wilbois, Few-Body Syst. (in print)

[RiB 72] D.O. Riska and G.E. Brown, Phys. Lett. 38B, 193 (1972)

[RiG 97] F. Ritz, H. Göller, T. Wilbois, and H. Arenhövel, Phys. Rev. C 55, 2214 (1997)

[SaA 51] R.G. Sachs and N. Austern, Phys. Rev. 81, 705 (1951)

[Sai 69] S. Saito, Phys. Rev. 184, 1894 (1969)

[SAID] SAID: Scattering Analysis Interactive Dial-in programm via telnet to vtinte.phys.vt.edu.

[Sau 86] P.U. Sauer, Prog. Part. Nucl. Phys. 16, 35 (1986)

[SaF 95] Ch. Sauermann, B.L. Friman, W. Nörenberg, Phys. Lett. B 341, 261 (1995)

[SaW 94] A.M. Sandorfi, C.S. Whisnant, and M. Khandaker, Phys. Rev. D 50, R6681 (1994)

[ScA 96] R. Schmidt, H. Arenhövel, and P. Wilhelm, Z. Phys. A 355, 421 (1996)

[ScA 97] M. Schwamb, H. Arenhövel, P. Wilhelm, and Th. Wilbois, Phys. Lett. B (in print)

[ScW 90] K.-M. Schmitt, P. Wilhelm, H. Arenhövel, A. Cambi, B. Mosconi, and P. Ricci, Phys. Rev. C 41, 841 (1990)

[ScW 96] M. Schwamb, H. Arenhövel, and P. Wilhelm, Few-Body Syst. 19, 121 (1995)

[SiB 79] G.G. Simon et al., Nucl. Phys. A 324, 277 (1979)

[Sie 37] A.J.F. Siegert, Phys. Rev. 52, 787 (1937)

[SuM 58] ～E.C.G. Sudarshan and R.E. Marshak, Phys. Rev. 109, 1860 (1958)

[TaO 85] H. Tanabe and K. Ohta, Phys. Rev. C 31, 1876 (1985)

[Wey 29] H. Weyl, Z. Phys. 56, 330 (1929)

[WeA 78] H.J. Weber and H. Arenhövel, Phys. Rep. 36, 279 (1978)

[Wei 95] S. Weinberg, The Quantum Theory of Fields (Cambridge University Press 1995)

[WiA 93] P. Wilhelm and H. Arenhövel, Phys. Lett. B 318, 410 (1993)

[WiA 95] P. Wilhelm and H. Arenhövel, Nucl. Phys. A 593, 435 (1995)

[WiA 96] P. Wilhelm and H. Arenhövel, Nucl. Phys. A 609, 469 (1996)

[WiW 96] P. Wilhelm, Th. Wilbois, and H. Arenhövel, Phys. Rev. C 54, 1423 (1996)

[WiW 98] Th. Wilbois, P. Wilhelm, and H. Arenhövel, Phys. Rev. C 57, 295 (1998)

[WiB 93] Th. Wilbois, G. Beck, and H. Arenhövel, Few-Body Syst. 15, 39 (1993)

[WuA 57] C.S. Wu, E. Ambler, R.W. Hayward, D. Hoppes, and R. Hudson, Phys. Rev. 105, 1413 (1957)

[YaM 54] C.N. Yang and R. Mills, Phys. Rev. 96, 191 (1954)

[Yuk 35] H. Yukawa, Proc. Phys.-Math. Soc. Jap. 17, 48 (1935) 